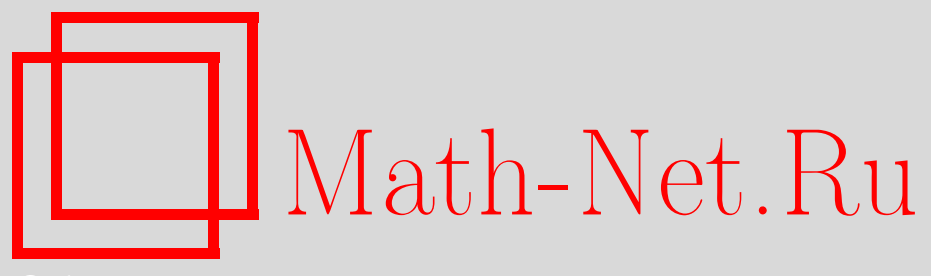

А. М. Левин, М. А. Ольшанецкий, А. В. Зотов, Классификация изомонодромных задач на эллиптических кривых, УМH, 2014, том 69, выпуск 1, 39-124

DOI: https://doi.org/10.4213/rm9576

Использование Общероссийского математического портала Math-Net.Ru подразумевает, что вы прочитали и согласны с пользовательским соглашением http://www . mathnet.ru/rus/agreement

Параметры загрузки:

IP : 52.90 .164 .192

26 апреля 2023 г., $07: 06: 20$

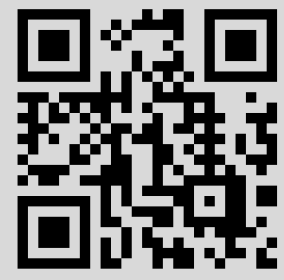




\title{
Классификация изомонодромных задач на эллиптических кривых
}

\author{
А. М. Левин, М. А. Ольшанецкий, А. В. Зотов
}

\begin{abstract}
В данной работе изомонодромные задачи описываются в терминах плоских $G$-расслоений на проколотых эллиптических кривых $\Sigma_{\tau}$ и связностей с регулярными особенностями в отмеченных точках. Расслоения классифицируются по их характеристическим классам, которые являются элементами группы вторых когомологий $H^{2}\left(\Sigma_{\tau}, \mathscr{Z}(G)\right)$, где $\mathscr{Z}(G)$ - центр $G$. По каждой простой комплексной группе Ли $G$ и произвольному характеристическому классу определяется пространство модулей плоских связностей, на которых уравнения изомонодромных деформаций задаются в гамильтоновой форме вместе с соответствующим представлением Лакса. Описываемые семейства задач включают в себя уравнение Пенлеве VI, его многокомпонентные обобщения и эллиптические системы Шлезингера. Общая конструкция описана для проколотой комплексной кривой произвольного рода. Описание Дринфельда-Симпсона пространства модулей расслоений Хиггса в виде двойного факторпространства обобщается на случай пространства плоских связностей. Такое локальное описание позволяет задать симплектическое соответствие Гекке для широкого круга изомонодромных задач, классифицируемых характеристическими классами отвечающих им расслоений. Например, уравнение Пенлеве VI описывается в терминах $\mathrm{SL}(2, \mathbb{C})$-расслоений. Так как $\mathscr{Z}(\mathrm{SL}(2, \mathbb{C}))=\mathbb{Z}_{2}$, то это уравнение имеет два представления, связанных преобразованием Гекке: 1) в виде широко известной эллиптической формы уравнения Пенлеве VI (для тривиальных расслоений); 2) в виде неавтономного гиростата Жуковского-Вольтерра (для нетривиальных расслоений).
\end{abstract}

Библиография: 123 названия.

Ключевые слова: изомонодромные деформации, уравнения Пенлеве, системы Шлезингера, расслоения Хиггса, плоские связности.

DOI: $10.4213 / \mathrm{rm} 9576$

Работа выполнена при поддержке РФФИ (грант № 12-02-00594) и молодежного проекта 12-01-33071_мол_а_вед, а также программы "Ведущие научные школы" (грант НШ-4724.2014.2). Первый автор поддержан также Лабораторией алгебраической геометрии НИУ "ВШЭ" (грант Правительства РФ, дог. 11.G34.31.0023). Третий автор поддержан также фондом Дмитрия Зимина “Династия". 


\section{СоДЕРЖАНИЕ}

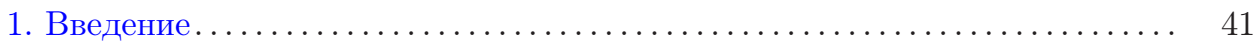

Глава I. Общий подход к изомонодромной задаче .................. 45

2. Плоские расслоения. Общий случай........................ 45

2.1. Пространство модулей плоских расслоений на гладких кривых . 45

2.2. Модули плоских расслоений с квазипараболической структурой 47

3. Плоские расслоения и характеристические классы ............... 49

3.1. Модули голоморфных расслоений в описании Дринфельда-Симп-

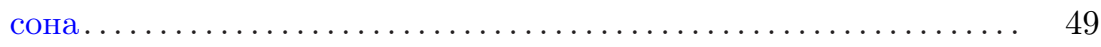

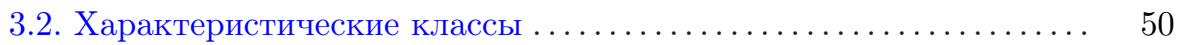

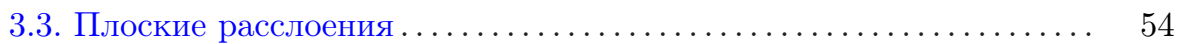

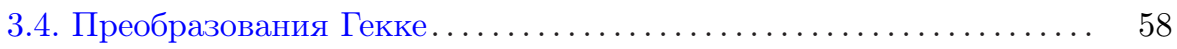

4. Гамильтонов подход к изомонодромной задаче ................ 60

4.1. Деформация комплексных структур на кривых ............. 60

4.2. Уравнения движения и изомонодромная задача ............ 62

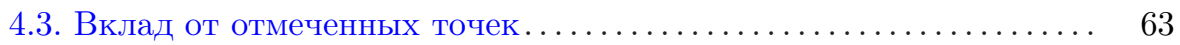

4.4. Симплектическая редукция .......................... 64

4.5. Изомонодромные деформации и интегрируемые системы ...... 66

Глава II. Изомонодромные задачи на эллиптических кривых . . . . . . . . . 66

5. Модули плоских расслоений на эллиптических кривых . . . . . . . . . 66

5.1. Голоморфные расслоения на эллиптических кривых ......... 67

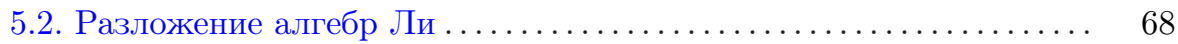

5.3. Пространство модулей голоморфных расслоений ............ 69

6. Изомонодромные уравнения на эллиптических кривых . . . . . . . . . . 72

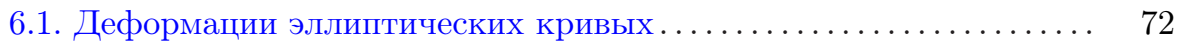

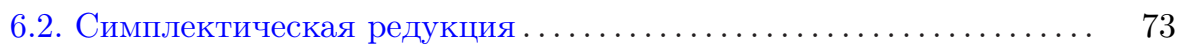

6.3. Матрица Лакса........................................ 74

6.4. Классическая $r$-матрица .......................... 76

6.5. Симметрии фазового пространства ..................... 77

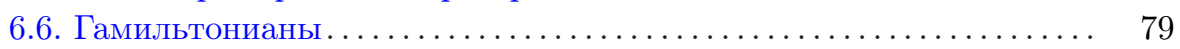

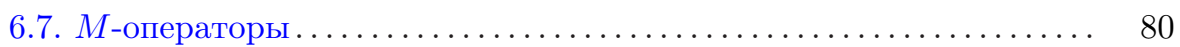

6.8. Уравнения Пенлеве-Шлезингера ....................... 81

6.9. Уравнения Книжника-Замолодчикова-Бернара............. 82

6.10. Теория поля Пенлеве............................... 84

7. Симплектическое соответствие Гекке ...................... 85

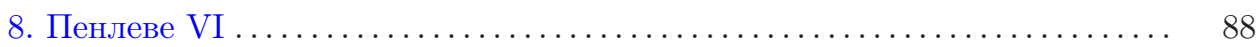

8.1. Три формы уравнения Пенлеве VI................... 88

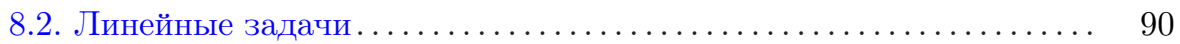

8.3. Эллиптическая форма рациональной связности ............. 92

8.4. Симплектическое соответствие Гекке .................... 93

Приложение А. Простые группы Ли.......................... 94

Приложение В. Обобщенный синус-базис в простых алгебрах Ли........ 103

Приложение С. Эллиптические функции . . . . . . . . . . . . . . . . 110

Приложение D. Характеристические классы и конформные группы ..... 113

Список литературы ....................................... 117 


\section{1. Введение}

В настоящей работе мы предлагаем классификацию изомонодромных задач для плоских $G$-расслоений на эллиптических кривых $\Sigma_{\tau}=\mathbb{C} /(\mathbb{Z}+\tau \mathbb{Z})$ с логарифмическими особенностями в отмеченных точках $z_{a}, a=1, \ldots, n$. Группа Ли $G$ простая и комплексная. Напомним вкратце, что изомонодромная задача на комплексной кривой $\Sigma_{g}$ рода $g$ с $n$ отмеченными (проколотыми) точками приводит к следующим (сохраняющим монодромию или изомонодромным) уравнениям:

$$
\partial_{t_{j}} L(z)-\partial_{z} M_{j}(z)=\left[L(z), M_{j}(z)\right]
$$

где $z$ - локальная координата на $\Sigma_{g}, t_{j}$ - модули $\Sigma_{g, n}=\Sigma_{g} \backslash\left\{z_{1}, \ldots, z_{n}\right\}$ $(j=1, \ldots, 3 g-3+n$ для $g>1)$, а $L(z)$ и $M(z)$ суть $\mathfrak{g}=\operatorname{Lie}(G)$-значные функции. Уравнения (1.1) являются условиями совместности следующих линейных задач:

$$
\left\{\begin{array}{l}
\left(\partial_{z}+L(z)\right) \Psi=0, \\
\left(\partial_{t_{j}}+M_{j}(z)\right) \Psi=0,
\end{array} \quad \Psi=\left\|\psi_{1} \cdots \psi_{\operatorname{dim} V}\right\|,\right.
$$

где $V$ - конечномерный $G$-модуль, а $\psi_{k} \in \Gamma\left(E_{G}\right), k=1, \ldots, \operatorname{dim} V,-$ сечения векторного расслоения $E_{G}=\mathscr{P} \times_{G} V$, ассоциированного с главным $G$-расслоением $\mathscr{P}$. Нижний набор уравнений возникает из требования независимости монодромий решений верхних уравнений от модулей кривой $\Sigma_{g, n}$ при обходе вокруг отмеченных точек и фундаментальных циклов кривой $\Sigma_{g}$.

Уравнения (1.1) выполняются тождественно по $z$ и описывают конечномерные неавтономные гамильтоновы системы. В такой форме (Лакса или уравнения нулевой кривизны) представляются уравнения Пенлеве, системы Шлезингера и их обобщения. Исторически исследование изомонодромных задач было мотивировано открытием списка уравнений Пенлеве [1]-[3]. Оказалось, что нелинейные обыкновенные дифференциальные уравнения из этого списка могут быть записаны в форме условия совместности пары линейных уравнений на проколотой сфере Римана [4]-[6]. Этот результат был переоткрыт в окрестности 1980-х [7]-[11]. Сейчас существует обширная литература, посвященная уравнениям Пенлеве и их многочисленным приложениям в математической физике [12]-[24].

Целью работы является классификация уравнений (1.1), (1.2) для случая $g=1$, т. е. для эллиптических кривых $\Sigma_{\tau}$. Изомонодромные задачи на компактных римановых поверхностях и, в частности, на торе исследовались аналитическими методами в работах [25]-[27]. Мы будем пользоваться подходом, предложенным в [28]-[30]. Он в свою очередь основан на неавтономной версии систем Хитчина [31] на проколотых эллиптических кривых [32]-[35] (см. также [36]-[38]). Близкая по духу конструкция уравнений Пенлеве была предложена в [39]. Идея состояла в редукции пространства плоских связностей по действию калибровочной группы симметрий $\mathscr{G}$, генерируемой автоморфизмами $E_{G}$, к их пространству модулей. Отображение момента приводит к уравнению на $L(z)$ следующего вида:

$$
\bar{\partial} L(z)=\sum_{a=1}^{n} S^{a} \delta^{(2)}\left(z-z_{a}\right),
$$


где $S^{a}$ - вычеты $L(z)$ в отмеченных точках. Уравнение (1.3) вместе с граничными условиями для решений (1.1)

$$
\Psi(z+1)=\mathscr{Q}(z) \Psi(z), \quad \Psi(z+\tau)=\Lambda(z) \Psi(z)
$$

фиксирует $L(z)$. Матрицы $\mathscr{Q}(z)$ и $\Lambda(z)$ - функции переклейки расслоения $E_{G}$. Они удовлетворяют условию коцикла

$$
\mathscr{Q}(z) \Lambda^{-1}(z) \mathscr{Q}^{-1}(z+\tau) \Lambda(z+1)=1 .
$$

Отметим еще раз, что те же уравнения (1.3)-(1.5) описывают и интегрируемые системы (Хитчина) на эллиптической кривой. Их решения (в некоторых частных случаях) известны как эллиптические системы Годена [40], [32]. Соответствующие матрицы $L(z)$ были построены естественным обобщением анзаца Кричевера пар Лакса для эллиптических интегрируемых систем [41]. Фазовые пространства для указанных систем состоят из двух частей: набора $S^{a}-$ “спиновой” части и модулей решений (1.5) - конфигурационного пространства, связанного со степенями свободы многочастичной динамики. Изомонодромные версии интегрируемых систем включают в себя неавтономные обобщения эллиптических систем Калоджеро, эллиптического волчка и эллиптических систем Шлезингера [30], [42], [43]. Явление, состоящее в том, что одна и та же пара матриц удовлетворяет как уравнению Лакса для интегрируемой системы, так и уравнению изомонодромных деформаций (1.1), было обнаружено в [44] и известно как соответствие Калоджеро-Пенлеве.

В общем случае решения (1.5) классифицируются характеристическими классами расслоений, т. е. элементами группы вторых когомологий $H^{2}\left(\Sigma_{\tau}, \mathscr{Z}(G)\right)$, где $\mathscr{Z}(G)$ - центр $G$. Классификация таких решений, соответствующих расслоений Хиггса и интегрируемых систем (в случае единственной отмеченной точки) описана в наших недавних работах [45], [46] (см. также [47]-[49]). Классификация возникает из того факта, что функции переклейки $\mathscr{Q}(z)$ и $\Lambda(z)$ могут быть выбраны $z$-независимыми с точностью до скалярного множителя $(\mathscr{Q}$ и $\Lambda$ ). Тогда уравнение (1.5) переходит (с учетом упомянутого скалярного множителя) в

$$
\mathscr{Q} \Lambda^{-1} \mathscr{Q}^{-1} \Lambda=\zeta, \quad \zeta \in \mathscr{Z}(G) .
$$

Наиболее репрезентативными случаями для $\operatorname{SL}(N, \mathbb{C})$-расслоений ${ }^{1}$ являются $\zeta=1$ и $\zeta=\exp (2 \pi \sqrt{-1} / N)$. Отвечающие этим случаям интегрируемые системы - это эллиптическая модель Калоджеро-Мозера и эллиптический волчок соответственно. Нетривиальные промежуточные случаи возникают, когда $N=p l$. При этом характеристические классы, равные $\zeta=\exp ((2 \pi \sqrt{-1} / N) p)$ или $\zeta=\exp ((2 \pi \sqrt{-1} / N) l)$, отвечают так называемым моделям взаимодействующих волчков [51], [52]. Размерность фазового пространства остается такой же, как и в случае системы Калоджеро со спином, но с меньшим числом частиц $(n<N)$ и большим числом спиновых степеней свободы.

\footnotetext{
${ }^{1}$ Классификация расслоений на эллиптических кривых была предложена М. Атьей [50]. В наших терминах его результат выглядит так: $H^{2}\left(\Sigma_{\tau}, \mathscr{Z}(\operatorname{SL}(N, \mathbb{C}))\right) \sim \mathscr{Z}(\operatorname{SL}(N, \mathbb{C}))=\mathbb{Z} / N \mathbb{Z}$, т. е. расслоения классифицируются корнем $N$-й степени из единицы.
} 
В настоящей работе мы предлагаем обобщение классификации расслоений Хиггса и интегрируемых систем по характеристическим классам [45], [46] $]^{2}$ на случай плоских связностей и произвольного числа отмеченных точек на эллиптической кривой.

Мы используем два описания пространства модулей плоских связностей. Первое из них [30] является естественным обобщением теоретико-группового описания пространства модулей расслоений Хиггса в рамках подхода Хитчина к интегрируемым системам [32], [35]:

$$
\operatorname{FBun}\left(\Sigma_{g}, G\right)=\operatorname{Conn}\left(\Sigma_{g}, G\right) / / \mathscr{G}=\operatorname{FConn}\left(\Sigma_{g}, G\right) / \mathscr{G} .
$$

Оно получается как факторпространство плоских связностей

$$
\operatorname{FConn}\left(\Sigma_{g}, G\right)=\left\{d+\mathscr{A} \mid d \mathscr{A}+\frac{1}{2} \mathscr{A} \wedge \mathscr{A}=0\right\}
$$

по калибровочной группе $\mathscr{G}$. Пространство $\operatorname{Conn}\left(\Sigma_{g}, G\right)=\{d+\mathscr{A}\}$ гладких связностей на $E_{G}$ снабжено хорошо известной симплектической структурой (Атьи-Ботта) $\omega=\frac{1}{2} \int_{\Sigma_{g}}\langle\delta \mathscr{A} \wedge \delta \mathscr{A}\rangle$ [56]. После редукции она становится симплектической структурой конечномерной гамильтоновой системы. Его фазовое пространство $\operatorname{FBun}\left(\Sigma_{g}, G\right)$ - главное однородное пространство $\operatorname{PT}^{*} \operatorname{Bun}\left(\Sigma_{g}, G\right)$ над $T^{*} \operatorname{Bun}\left(\Sigma_{g}, G\right)$.

Второе описание пространства $\operatorname{FBun}\left(\Sigma_{g}, G\right)$ происходит из описания Дринфельда-Симпсона [57], [58] для модулей расслоений Хиггса. Оно формулируется в терминах локальных данных (см. раздел 3). Пространство модулей плоских расслоений с квазипараболическими структурами в отмеченных точках представляется в виде двойного факторпространства:

$$
\operatorname{Bun}\left(\Sigma_{g, n}, G\right)=G\left(\Sigma_{g, n}\right) \backslash G\left(D^{\times}\right) / G(D),
$$

где $G(X)$ - голоморфные отображения из $X \subset \Sigma_{g}$ в $G, D^{\times}$- дизъюнктное объединение малых проколотых дисков в окрестностях отмеченных точек (где расслоение тривиализуется), а $G(D)$ - специальные отображения, сохраняющие флаги, отвечающие квазипараболическим структурам в отмеченных точках. На уровне $\operatorname{FBun}\left(\Sigma_{g}, G\right)$ эта конструкция как раз и означает, что мы работаем с тривиальными расслоениями на $\Sigma_{g, n}$ с регулярными особенностями в отмеченных точках. Как известно, регулярную особенность можно перевести в фуксову некоторым мероморфным калибровочным преобразованием на диске. Тем самым, естественно снабдить связность мероморфным калибровочным преобразованием, определенным с точностью до умножения справа на голоморфное на диске преобразование. Таким образом, действие $G\left(D^{\times}\right)$может преобразовать регулярную особенность в фуксову. Тогда такое мероморфное преобразование можно трактовать как функцию переклейки нетривиального

\footnotetext{
${ }^{2}$ Альтернативная классификация расслоений Хиггса была недавно предложена в [53]. Сравнение результатов с [45], [46] требует дальнейшего изучения. Некомпактный случай вещественных групп Ли обсуждался недавно в работах [54], [55].
} 
расслоения с фуксовой особенностью. Таким способом мы приходим к описанию $\operatorname{FBun}\left(\Sigma_{g}, G\right)$, аналогичному (1.9).

Последнее описание особенно удачно с точки зрения определения операторов Гекке (или модификаций расслоений) [59], [60], которые связывают линейные задачи (1.2) и уравнения (1.1) для различных характеристических классов. На уровне компоненты связности $L(z)$ модификация действует в виде калибровочного преобразования

$$
L \stackrel{\Xi}{\longrightarrow} L^{\bmod }, \quad L^{\bmod }(z) \Xi(z)=\Xi(z) L(z)-\partial_{z} \Xi(z),
$$

вырожденного в некоторой точке $z_{0}$, т. е. $\operatorname{det} \Xi\left(z, z_{0}\right) \sim z-z_{0}$ вблизи $z_{0}$. В этом смысле мы обобщаем симплектическое соответствие Гекке, определенное в [61], на случай уравнений изомонодромных деформаций. Рассмотрим в качестве примера уравнение Пенлеве VI. Оно описывается в терминах $\mathrm{SL}(2, \mathbb{C})$-расслоений. Так как $\mathscr{Z}(\mathrm{SL}(2, \mathbb{C}))=\mathbb{Z}_{2}$, то уравнение имеет два представления, связанные преобразованием Гекке:

1) эллиптическая форма уравнения Пенлеве VI [62], [63]:

$$
\frac{d^{2} u}{d \tau^{2}}=\sum_{a=0}^{3} \nu_{a}^{2} \wp^{\prime}\left(u+\omega_{a}\right)
$$

2) неавтономный аналог гиростата Жуковского-Вольтерра [64]:

$$
\partial_{\tau} S=[S, J(S)]+\left[S, \nu^{\prime}\right]
$$

где $S$ обозначает $\mathrm{sl}^{*}(2, \mathbb{C})$-значную динамическую переменную, а $J$ и $\nu^{\prime}-$ нединамические, но $\tau$-зависимые. Линейные $(2 \times 2)$-задачи для $(1.11)$ и (1.12) были описаны в [65] и [64] соответственно. Подробности изложены в разделе 8. В общем случае преобразование Гекке (1.10) можно рассматривать как преобразование Бэклунда для изомонодромных уравнений. Иными словами, это преобразование является аналогом сдвига по дискретному времени или аналогом преобразования Шлезингера [10].

Уравнения, сохраняющие монодромию, можно рассматривать как деформации интегрируемых систем. Интегрируемые семейства, отвечающие рассматриваемым задачам, были описаны в [45], [46]. Соответствие между интегрируемыми системами и уравнениями изомонодромных деформаций, названное в [44] соответствием Калоджеро-Пенлеве, использовалось еще П. Бутру при исследовании уравнений Пенлеве [66]. Аналогично Р. Гарнье построил автономные аналоги уравнений Шлезингера [67] и таким образом пришел к изоспектральной задаче. Г. Флашка и А. Ньюэлл в [7], [9], а также недавно И. Кричевер в [68] развили программу Бутру-Гарнье и обнаружили, что ВКБ-приближение по "постоянной Планка" $\kappa$ преобразует изомонодромную задачу в изоспектральную.

Статья состоит из двух глав и приложений. Первая глава посвящена общему подходу к изомонодромной задаче на $\Sigma_{g, n}$. В разделах 2 и 3 двумя способами описано пространство плоских связностей. Первый способ - в терминах связностей (1.8) и калибровочной группы. Второй - в терминах локальных 
данных, в виде двойного факторпространства (1.9). Последний используется для определения характеристических классов и преобразований Гекке в конце раздела 3. В разделе 4 описана сама изомонодромная задача и ее гамильтонова формулировка. Во второй главе детально исследуется случай эллиптической кривой. В ней мы обобщаем полученную нами ранее классификацию эллиптических интегрируемых систем на уравнения изомонодромных деформаций. Вкратце описываются и уравнения Книжника-Замолодчикова-Бернара, а также теоретико-полевые обобщения полученных уравнений. Классификация демонстрируется на примере уравнения Пенлеве VI (раздел 8). В приложениях мы приводим необходимый перечень сведений о группах Ли и эллиптических функциях. Также описан обобщенный базис синус-алгебры для алгебр Ли, используемый при классификации по характеристическим классам. Кроме того, в приложениях описаны конформные версии групп Ли, что позволяет естественным образом связать характеристические классы со степенями расслоений.

Мы выражаем признательность В. Побережному за полезные обсуждения.

\section{Глава I. Общий подход к изомонодромной задаче}

Пусть $G$ - простая комплексная группа Ли, а $E_{G}$ - плоское $G$-расслоение над кривой $\Sigma_{g, n}$ рода $g$ с $n$ отмеченными точками. Комплексная структура на $\Sigma_{g, n}$ задает поляризацию связностей, действующих на сечениях $\Gamma\left(E_{G}\right)$. Локально в комплексных координатах $(z, \bar{z})$ связность $d+\mathscr{A}$ имеет вид $((\partial+A) \otimes d z$, $(\bar{\partial}+\bar{A}) \otimes d \bar{z})$. Вследствие плоскости следующая система совместна:

$$
\left\{\begin{array}{l}
(\partial+A) \psi=0, \\
(\bar{\partial}+\bar{A}) \psi=0,
\end{array} \quad \psi \in \Gamma\left(E_{G}\right) .\right.
$$

Предположим, что монодромии решений $\psi$ не зависят от комплексной структуры $\Sigma_{g, n}$. Условием независимости являются дифференциальные уравнения, которые в некоторых случаях можно выписать явно. Основным объектом исследования будут $G$-расслоения над эллиптическими кривыми $\Sigma_{\tau}=\mathbb{C} /(\mathbb{Z}+\tau \mathbb{Z})$. Но прежде рассмотрим случай кривой произвольного рода.

\section{2. Плоские расслоения. Общий случай}

Пусть $\mathscr{P}$ - главное $G$-расслоение над $\Sigma_{g, n}, V$ - конечномерный модуль $G$ и $E_{G}=\mathscr{P} \times_{G} V$.

Рассмотрим два случая:

1) гладкая (компактная) кривая $(n=0)$;

2) гладкая кривая с проколами (отмеченными точками) $(n \neq 0)$.

2.1. Пространство модулей плоских расслоений на гладких кривых. Определим пространство $\operatorname{Conn}\left(\Sigma_{g}, G\right)=\{d+\mathscr{A}\}$ гладких связностей на $E_{G}$ для гладких кривых. Группа $\mathscr{G}$ автоморфизмов расслоения $E_{G}$ (или калибровочная группа) действует на связностях аффинными преобразованиями:

$$
\mathscr{G}: \mathscr{A} \rightarrow f^{-1} d f+f^{-1} \mathscr{A} f .
$$


Пусть $\mathrm{FConn}\left(\Sigma_{g}, G\right)$ - пространство плоских связностей:

$$
\operatorname{FConn}\left(\Sigma_{g}, G\right)=\left\{d+\mathscr{A} \mid d \mathscr{A}+\frac{1}{2} \mathscr{A} \wedge \mathscr{A}=0\right\} .
$$

Тогда пространство модулей плоских связностей - это факторпространство

$$
\operatorname{FBun}\left(\Sigma_{g}, G\right)=\operatorname{FConn}\left(\Sigma_{g}, G\right) / \mathscr{G} .
$$

С другой стороны, $\operatorname{FBun}\left(\Sigma_{g}, G\right)$ можно представить как результат гамильтоновой редукции симплектического пространства $\operatorname{Conn}\left(\Sigma_{g}, G\right)$ всех гладких связностей по действию калибровочной группы $\mathscr{G}$. Симплектическая форма на $\operatorname{Conn}\left(\Sigma_{g}, G\right)$ есть

$$
\omega=\frac{1}{2} \int_{\Sigma_{g}}\langle\delta \mathscr{A} \wedge \delta \mathscr{A}\rangle,
$$

где $\langle\cdot, \cdot\rangle$ обозначает форму Киллинга, $\delta \mathscr{A}$ есть $\operatorname{Lie}(G)$-значная 1-форма на $\Sigma_{g}$. То есть $\langle\delta \mathscr{A}, \delta \mathscr{A}\rangle$ является 2-формой, и интеграл корректно определен. Эта форма калибровочно инвариантна. Гамильтонова редукция по действию группы дает отображение момента

$$
\begin{aligned}
\operatorname{Conn}\left(\Sigma_{g}, G\right) & \rightarrow \mathscr{G}^{*} \sim \Omega_{C^{\infty}}^{2}\left(\Sigma_{g}, \operatorname{Lie}^{*}(G)\right) \\
\mathscr{A} & \mapsto F_{\mathscr{A}}=d \mathscr{A}+\mathscr{A} \wedge \mathscr{A} .
\end{aligned}
$$

Тем самым, прообраз нуля при отображении момента - это пространство плоских связностей $\mathrm{FConn}\left(\Sigma_{g}, G\right)(2.2)$. Выбор комплексной структуры на $\Sigma_{g}$ задает поляризацию $\operatorname{Conn}\left(\Sigma_{g}, G\right)$. Тогда связность раскладывается на $(1,0)$ и $(0,1)$-части: $\mathscr{A}=(A, \bar{A})$. Компоненты связности $((1,0)$ и $(0,1))$ можно записать в локальных координатах $(z, \bar{z})$ :

$$
\operatorname{Conn}\left(\Sigma_{g}, G\right)=\left\{d^{\prime}=(\partial+A) \otimes d z, d^{\prime \prime}=(\bar{\partial}+\bar{A}) \otimes d \bar{z}\right\} \quad\left(\partial=\partial_{z}, \bar{\partial}=\partial_{\bar{z}}\right)
$$

В таком описании $\omega(2.4)$ принимает вид

$$
\omega=\int_{\Sigma_{g}}\langle\delta A \wedge \delta \bar{A}\rangle .
$$

Определим пространство модулей голоморфных расслоений. Сечение $s \in \Gamma\left(E_{G}\right)$ является голоморфным, если оно зануляется оператором $\bar{\partial}+\bar{A}$. Модули голоморфных расслоений задаются факторпространством

$$
\operatorname{Bun}\left(\Sigma_{g}, G\right)=\{\bar{\partial}+\bar{A}\} / \mathscr{G} .
$$

Пусть $b$ - точка в $\operatorname{Bun}\left(\Sigma_{g}, G\right)$, а $\mathscr{V}_{b}$ - отвечающее ей $E_{G}$-расслоение на $\Sigma_{g}$. Обозначим через $\mathrm{ad}_{b}$ расслоение эндоморфизмов $\mathscr{V}_{b}$ со слоями, изоморфными алгебре Ли. Пусть $\mathrm{Flat}_{b}$ - пространство плоских голоморфных связностей на голоморфном расслоении $\mathscr{V}_{b}$. Иными словами, в локальных координатах

$$
\text { Flat }_{b}=\{\partial+A \mid F(A, \bar{A})=0\} .
$$


Пространство, касательное к $\operatorname{Bun}\left(\Sigma_{g}, G\right)$ в точке $b=E_{G}$, канонически изоморфно группе первых когомологий $H^{1}\left(\Sigma_{g}, \mathrm{ad}_{b}\right)$. Пространство Flat морфных связностей на $\mathscr{V}_{b}$ является аффинным пространством над векторным пространством $H^{0}\left(\Sigma_{g}, \operatorname{ad}_{b} \otimes \Omega^{1}\right)$ голоморфных $\operatorname{ad}_{b}$-значных 1-форм, так как разность между парой связностей является $\mathrm{ad}_{b}$-значной дифференциальной формой. Векторные пространства $H^{1}\left(\Sigma_{g}, \operatorname{ad}_{b}\right)$ и $H^{0}\left(\Sigma_{g}, \operatorname{ad}_{b} \otimes \Omega^{1}\right)$ двойственны.

Рассмотрим отображение из $\operatorname{FBun}\left(\Sigma_{g}, G\right)$ на пространство $\operatorname{Bun}\left(\Sigma_{g}, G\right)$. Слой проекции $\operatorname{FBun}\left(\Sigma_{g}, G\right) \rightarrow \operatorname{Bun}\left(\Sigma_{g}, G\right)$ над точкой $b$ изоморфен Flat ${ }_{b}$ :

$$
p: \operatorname{FBun}\left(\Sigma_{g}, G\right) \stackrel{\text { Flat }_{b}}{\longrightarrow} \operatorname{Bun}\left(\Sigma_{g}, G\right) .
$$

Эти слои лагранжевы относительно $\omega$ (2.6). По теореме Римана-Роха имеем

$$
\begin{gathered}
\operatorname{dim}\left(\operatorname{Bun}\left(\Sigma_{g}, G\right)\right)=\operatorname{dim}\left(H^{1}\left(\Sigma_{g}, \operatorname{ad}_{b}\right)\right)=\operatorname{dim}(G)(g-1), \\
\operatorname{dim}\left(\operatorname{FBun}\left(\Sigma_{g}, G\right)\right)=2 \operatorname{dim}(G)(g-1) .
\end{gathered}
$$

Пусть $K$ - канонический класс $\Sigma_{g}$. Расслоением Хиггса называется пара

$$
\left(E_{G}, \Omega^{0}\left(\Sigma_{g}, \operatorname{End}\left(E_{G}\right) \otimes K\right)\right) .
$$

В локальных координатах оно представляется парой $(\bar{\partial}+\bar{A}, \Phi)$, где

$$
\Phi \in \Omega^{0}\left(\Sigma_{g}, \operatorname{End}\left(E_{G}\right) \otimes K\right)
$$

называется полем Хиггса. Расслоение Хиггса является симплектическим многообразием с симплектической формой

$$
\omega^{\text {higgs }}=\int_{\Sigma_{g}}\langle\delta \Phi \wedge \delta \bar{A}\rangle
$$

(см. (2.4)). Форма инвариантна относительно действия калибровочной группы $\Phi \rightarrow \operatorname{Ad}_{f} \Phi, \bar{A} \rightarrow f^{-1} \bar{\partial} f+f^{-1} \bar{A} f$. После симплектической редукции получаем кокасательное $T^{*} \operatorname{Bun}\left(\Sigma_{g}, G\right)$ расслоение к пространству модулей. Оно является фазовым пространством интегрируемых систем Хитчина [31].

\section{2. Модули плоских расслоений с квазипараболической структу-} рой. Рассмотрим теперь кривые $\Sigma_{g, n}$ с отмеченными точками $\mathbf{x}=\left(x_{1}, \ldots, x_{n}\right)$. Пусть $P_{a}(a=1, \ldots, n)$ - параболические подгруппы, $P_{a} \subset G$. Припишем отмеченным точкам многообразия $G$-флагов Flag $a G / P_{a}$. Тогда будем говорить, что $E_{G}$ обладает квазипараболической структурой (qр-структурой) [69]. Группа автоморфизмов такого расслоения $\mathscr{G}_{P}$ сохраняет флаги в отмеченных точках. Это означает, что в окрестности $\mathscr{U}_{a}$ точки $x_{a}$

$$
\mathscr{G}_{P}=\left\{f \in \mathscr{G}|f|_{\mathscr{U}_{a}}=P_{a}+O\left(z-x_{a}\right)\right\} .
$$

Заменим пространство модулей $\operatorname{Bun}\left(\Sigma_{g}, G\right)(2.7)$ на $\operatorname{Bun}\left(\Sigma_{g, n}, \mathbf{x}, G\right)$ для $G$-расслоений $E_{G}$ с qр-структурой,

$$
\operatorname{Bun}\left(\Sigma_{g, n}, \mathbf{x}, G\right)=\{\bar{\partial}+\bar{A}\} / \mathscr{G}_{P} .
$$


Существует естественная проекция “забывания":

$$
\pi: \operatorname{Bun}\left(\Sigma_{g}, \mathbf{x}, G\right) \stackrel{\Pi_{a} \operatorname{Flag}_{a}}{\longrightarrow} \operatorname{Bun}\left(\Sigma_{g}, G\right) .
$$

Расслоение эндоморфизмов, построенное по этим данным, - это расслоение эндоморфизмов $\operatorname{Lie}\left(\mathscr{G}_{P}\right)=\operatorname{ad}_{b, \operatorname{Flag}}(-\mathbf{x})$, сечения которого в окрестности отмеченных точек ведут себя следующим образом:

$$
\varphi\left(z-x_{a}\right)=\varphi_{a}^{0}+\left(z-x_{a}\right) \varphi_{a}^{1}+\cdots, \quad \varphi_{a}^{0} \in \operatorname{Lie}\left(P_{a}\right) .
$$

Касательное пространство к $\operatorname{Bun}\left(\Sigma_{g}, \mathbf{x}, G\right)$ изоморфно $H^{1}\left(\Sigma_{g}, \operatorname{ad}_{b, \mathrm{Flag}}(-\mathbf{x})\right)$. Тогда двойственным к нему является $H^{0}\left(\Sigma, \operatorname{ad}_{b, \text { Flag }}^{*}(\mathbf{x}) \otimes \Omega^{1}\right)$.

Рассмотрим коприсоединенные $G$-орбиты в коалгебре Ли $\mathfrak{g}^{*}$, помещенные в отмеченные точки:

$$
\mathscr{O}_{a}=\left\{\mathbf{S}_{a}=\operatorname{Ad}_{g} S_{a}^{0}, g \in G, S_{a}^{0} \in \mathfrak{g}^{*}\right\} \quad(a=1, \ldots, n) .
$$

Переменные $\mathbf{S}_{a}$ называются спиновыми. Орбита $\mathscr{O}$ расслаивается над многообразием флагов Flag, а слоями являются однородные пространства $P T^{*}$ Flag над кокасательными расслоениями $T^{*}$ Flag. Это симплектическое пространство с симплектической структурой Кириллова-Костанта:

$$
\omega^{\mathrm{KK}}=\delta\left\langle S^{0}, \delta g g^{-1}\right\rangle=\left\langle S, g^{-1} \delta g \wedge g^{-1} \delta g\right\rangle .
$$

Рассмотрим пространство гладких связностей $\operatorname{Conn}\left(\Sigma_{g, n} G\right)$ с особенностями в отмеченных точках. В малых окрестностях точек $\mathscr{U}_{a}$ связность $\bar{A}$ можно откалибровать на ноль $(\bar{A}=0)$. Предположим, что $A$ имеет (голоморфные) полюсы первого порядка в $x_{a}$ с вычетами, принимающими значения в $\mathscr{O}_{a}$. Тогда пространство связностей определяется как

$$
\operatorname{Conn}\left(\Sigma_{g, n}, G\right)=\left\{\begin{array}{l|l}
(A, \bar{A}) & \begin{array}{l}
\left.\bar{A}\right|_{\mathscr{U}_{a}}=0 \\
A=\mathbf{S}_{a}\left(z_{a}-x_{a}\right)^{-1}+O(1), \mathbf{S}_{a} \in \mathscr{O}_{a}
\end{array}
\end{array}\right\} .
$$

Условие (2.2) того, что связности являются плоскими, учитывает теперь наличие полюсов:

$$
\operatorname{FConn}\left(\Sigma_{g, n}, G\right)=\left\{\mathscr{A} \in \operatorname{Conn}\left(\Sigma_{g, n}, G\right) \mid F_{\mathscr{A}}=\sum_{a=1}^{n} \mathbf{S}_{a} \delta_{x_{a}}^{(2)}\right\}
$$

Следовательно, для получения симплектического многообразия нужно заменить аффинное пространство $\mathrm{Flat}_{b}$ плоских голоморфных связностей на аффинное пространство $\mathrm{Flat}_{b}(\log )$ плоских голоморфных связностей с логарифмическими особенностями. В итоге получаем пространство модулей (см. (2.3)) пар (голоморфное расслоение, голоморфная связность с логарифмическими особенностями и вычетами в виде орбит $\mathscr{O}_{a}$ в отмеченных точках):

$$
\operatorname{FBun}\left(\Sigma_{g}, \mathbf{x}, G\right)=\operatorname{FConn}\left(\Sigma_{g, n}, G\right) / \mathscr{G}_{P}=\operatorname{Conn}\left(\Sigma_{g, n}, G\right) / / \mathscr{G}_{P}
$$


Симплектическая форма (2.6) теперь есть

$$
\omega=\int_{\Sigma_{g}}\langle\delta A \wedge \delta \bar{A}\rangle+\sum_{a=1}^{n} \omega_{a}^{\mathrm{KK}} .
$$

Аналогично (2.14), имеем проекцию

$$
\pi: \operatorname{FBun}\left(\Sigma_{g}, \mathbf{x}, G\right) \stackrel{\Pi_{a} \mathscr{O}_{a}}{\longrightarrow} \operatorname{FBun}\left(\Sigma_{g}, G\right)
$$

и лагранжеву проекцию (см. (2.9))

$$
p: \operatorname{FBun}\left(\Sigma_{g}, \mathbf{x}, G\right) \rightarrow \operatorname{Bun}\left(\Sigma_{g}, \mathbf{x}, G\right) .
$$

Слоем проекции является аффинное пространство над $H^{0}\left(\Sigma, \operatorname{ad}_{b, \mathrm{Flag}}^{*}(\mathbf{x}) \otimes \Omega^{1}\right)$. Из (2.10) получаем:

$$
\begin{aligned}
\operatorname{dim}\left(\operatorname{Bun}\left(\Sigma_{g}, \mathbf{x}, G\right)\right) & =\operatorname{dim}(G)(g-1)+\sum_{a=1}^{n} \operatorname{dim}\left(\text { Flag }_{a}\right), \\
\operatorname{dim}\left(\operatorname{FBun}\left(\Sigma_{g}, \mathbf{x}, G\right)\right) & =2 \operatorname{dim}(G)(g-1)+\sum_{a=1}^{n} \operatorname{dim}\left(\mathscr{O}_{a}\right) .
\end{aligned}
$$

В частности, отсюда следует, что для нетривиальности пространства модулей расслоений над эллиптической кривой требуется хотя бы одна отмеченная точка.

\section{3. Плоские расслоения и характеристические классы}

\section{1. Модули голоморфных расслоений в описании Дринфельда-} Симпсона. В дальнейшем нам понадобится и другое описание для пространств $\operatorname{Bun}\left(\Sigma_{g, n}, \mathbf{x}, G\right)$ и $\operatorname{FBun}\left(\Sigma_{g, n}, \mathbf{x}, G\right)$. Они определяются следующим образом. $G$-расслоение тривиализуется над дизъюнктным объединением $D=\bigcup_{a=1}^{n} D_{a}$ малых дисков вокруг отмеченных точек, равно как и над $\Sigma_{g, n} \backslash \mathbf{x}$. Тогда $E_{G}$ определяется голоморфными функциями переклейки на $D^{\times}=\bigcup_{a=1}^{n} D_{a}^{\times}$, где $D_{a}^{\times}=D_{a} \backslash x_{a}$. Если $G(X)$ - голоморфные отображения из $X \subset \Sigma_{g}$ в $G$, то классы изоморфизма голоморфных расслоений задаются как двойные факторпространства

$$
\operatorname{Bun}\left(\Sigma_{g}, G\right)=\mathscr{G}_{\text {out }} \backslash G\left(D^{\times}\right) / \mathscr{G}_{\text {int }}, \quad \mathscr{G}_{\text {out }}=G\left(\Sigma_{g} \backslash \mathbf{x}\right), \quad \mathscr{G}_{\text {int }}=G(D) .
$$

Здесь $G\left(D^{\times}\right)$и $G(D)$ заданы как произведения групп петель. Пусть $t_{a}$ - локальные координаты в дисках $D_{a}$. Заменим $G(D)$ и $G\left(D^{\times}\right)$формальными рядами:

$$
\begin{gathered}
G(D)=\prod_{a=1}^{n} G\left(D_{a}\right) \rightarrow \prod_{a=1}^{n} G \otimes \mathbb{C}\left[\left[t_{a}\right]\right] \\
\left.G\left(D^{\times}\right) \rightarrow \prod_{a=1}^{n} L_{a}(G), \quad L_{a}(G)=G \otimes \mathbb{C}\left[t_{a}^{-1}, t_{a}\right]\right]
\end{gathered}
$$


Факторпространство снова изоморфно $\operatorname{Bun}\left(\Sigma_{g}, G\right)$ :

$$
\operatorname{Bun}\left(\Sigma_{g}, G\right)=\mathscr{G}_{\text {out }} \backslash \prod_{a=1}^{n} L_{a}(G) / \mathscr{G}_{\text {int }}, \quad \mathscr{G}_{\text {out }}=G\left(\Sigma_{g} \backslash \mathbf{x}\right), \quad \mathscr{G}_{\text {int }}=\prod_{a=1}^{n} G \otimes \mathbb{C}\left[\left[t_{a}\right]\right]
$$

Связность $d^{\prime \prime}(2.5)$ можно воссоздать по голоморфным функциям переклейки $g_{a} \in L_{a}(G)$. Тогда двойное факторпространство (3.3) эквивалентно определению (2.7).

Зафиксируем $G$-флаги в слоях над отмеченными точками. Напомним, что наличие qр-структуры $G$-расслоения означает, что $G(D)$ сохраняет эти $G$-флаги. Иными словами, $\mathscr{G}_{\mathrm{int}, P}=\prod_{a=1}^{n} L_{a}^{+}(G)$, где

$$
L_{a}^{+}(G)=\left\{g_{0}+g_{1} t+\cdots, g_{0} \in P\right\} \quad(\text { A. } 61)
$$

Пространство модулей голоморфных расслоений с qр-структурами в отмеченных точках есть двойное факторпространство

$$
\operatorname{Bun}\left(\Sigma_{g, n}, \mathbf{x}, G\right)=G\left(\Sigma_{g} \backslash \mathbf{x}\right) \backslash G\left(D^{\times}\right) / \mathscr{G}_{\text {int }, P}
$$

Ниже будет показано, что это определение эквивалентно предыдущему определению (2.13).

3.2. Характеристические классы. Для простоты рассмотрим сначала в (3.2) случай одной точки $\mathbf{x}=x_{0}$. Пусть $t-$ локальная координата в диске $D_{x_{0}}$ $\left(t=0 \sim x_{0}\right)$. В представлении (3.5) заменим $g(t) \in G\left(D^{\times}\right)$на $g(t) h(t)$, где $h(t) \in$ $D_{x_{0}}$. Вследствие $(3.5), h(t)$ определено с точностью до умножения на $f(t) \in$ $G(\mathbb{C}[[t]])$ справа. С другой стороны, так как исходная функция переклейки $g(t)$ определена с точностью до умножения справа на элемент из $G(\mathbb{C}[[t]])$, то $h(t)$ является элементом из двойного факторпространства

$$
L^{+}(G) \backslash L(G) / L^{+}(G)
$$

Для $g(t)=\widehat{w}=w t^{\gamma}$ это факторпространство есть аффинная клетка Шуберта $C_{\widehat{w}}(\mathrm{~A} .71)$ в аффинном многообразии флагов Flag ${ }^{\text {aff }}=L\left(G^{\text {ad }}\right) / L^{+}\left(G^{\text {ad }}\right)(3.6)$. Размерность $C_{\widehat{w}}$ равна $l(\widehat{w})($ см. $($ А.72)).

Вследствие (А.66)-(А.68), пространство модулей (3.5) является объединением секторов, определяемых аффинными группами Вейля. В частности, для $G^{\text {ad }}$-расслоений

$$
\begin{aligned}
\operatorname{Bun}\left(\Sigma_{g, n}, \mathbf{x}, G^{\mathrm{ad}}\right) & =\bigcup_{\widehat{w} \in \widetilde{W}_{P}} \operatorname{Bun}_{\widehat{w}}\left(\Sigma_{g, n}, \mathbf{x}, G^{\mathrm{ad}}\right), \\
\operatorname{Bun}_{\widehat{w}}\left(\Sigma_{g, n}, \mathbf{x}, G^{\mathrm{ad}}\right) & =G^{\mathrm{ad}}\left(\Sigma_{g} \backslash \mathbf{x}\right) \backslash G^{\mathrm{ad}}\left(D^{\times}\right) \widehat{w} / G^{\mathrm{ad}}(D) .
\end{aligned}
$$

Такое представление можно записать для $\bar{G}$ и $G_{l}$, определенной в (3.18). 
ПредлОжениЕ 3.1. Конструкция Дринфелъда-Симпсона для пространства модулей (3.7) эквивалентна его описанию по Дольбо (2.13).

ДокАзАтельство. Пусть $\left.g(t) \in G \otimes \mathbb{C}\left[t^{-1}, t\right]\right]$ - функция переклейки, задающая расслоение $E_{G}$. Рассмотрим разложение (А.68) функции $g(t)$ на малом диске $\widetilde{D}_{x_{0}} \supset D_{x_{0}}$ :

$$
\begin{gathered}
g(t)=g_{-} \widehat{w} g_{+}(t), \quad g_{-} \in N^{-}(G), \quad(\mathrm{A} .62), \\
g_{+}(t) \in G\left(D_{x_{0}}\right)=L^{+}(G), \quad(3.1) .
\end{gathered}
$$

Для $\mathbb{C P}^{1}$ оно совпадает с разложением Биркгофа (А.66)-(А.68) [70]. Это означает, что любое векторное расслоение $E_{G}$ на $\mathbb{C P}^{1}$ изоморфно прямой сумме линейных, $\bigoplus_{i=1}^{l} \mathscr{L}_{\gamma_{i}}$, где $\mathscr{L}_{\gamma_{i}}$ определяется функцией переклейки $t^{\gamma_{i}}, \gamma=$ $\left(\gamma_{1}, \ldots, \gamma_{l}\right) .^{3}$

Для описания пространства модулей в общем случае модифицируем конструкцию из работы [35], применявшуюся к описанию Чеха для $\operatorname{Bun}\left(\Sigma_{g}\right)$. Представим $g(t)(3.9)$ в виде

$$
g(t)=h_{\text {out }}^{-1} \widehat{w} h_{\text {int }} .
$$

Любое $g(t) \in L(G)$ (А.60) представляется в таком виде. В качестве примера рассмотрим разложение Брюа (А.68): $g(t)=g_{-} \widehat{w} g_{+}(t)$. Так как $N^{-}(G)-$ унипотентная группа, то ее логарифм $\log \left(g_{-}\right)$корректно определен. Заметим, что $g_{-}$и $\log \left(g_{-}\right)$являются голоморфными функциями на проколотом диске $\widetilde{D}_{x_{0}}^{\times}$. С помощью функции

$$
\chi(t, \bar{t})= \begin{cases}0, & t \notin \widetilde{D}_{x_{0}}, \\ 1, & t \in D_{x_{0}},\end{cases}
$$

определим (неголоморфное) продолжение функции $(3.9)$ из $\widetilde{D}_{x_{0}}^{\times}$в $\Sigma_{g}$ :

$$
g(t)=g_{-} \widehat{w} g_{+}(t) \rightarrow g(t)=h_{\text {out }}^{-1} \widehat{w} h_{\text {int }}, \quad h_{\text {int }}=g_{+}, \quad h_{\text {out }}=\exp \left(\chi(t, \bar{t}) \log \left(g_{-}\right)\right) .
$$

Такое представление имеет следующую интерпретацию. Пусть $\left(\mathbf{e}^{\text {hol }}\right)$ - базис в пространстве сечений тривиального голоморфного $G$-расслоения на $D_{x_{0}}$, a $\left(\mathbf{e}^{C^{\infty}}\right)$ - базис пространства сечений тривиального $G$-расслоения класса $C^{\infty}$ на $D_{x_{0}}$. Преобразование $h_{\text {int }}$ можно рассматривать как преобразование из $\left(\mathbf{e}^{\text {hol }}\right)$ в $\left(\mathbf{e}^{C^{\infty}}\right)$. Следовательно, $h_{\text {int }}$ определено в (3.12) с точностью до умножения справа на $g_{+}$и слева на $f_{\text {int }}$. Аналогичную роль играет и преобразование $h_{\text {out }}$. Будем называть такое представление неголоморфным разложением Биркгоба. Умножения справа на голоморфные преобразования

$$
h_{\mathrm{int}} \rightarrow h_{\mathrm{int}} g_{+}, \quad g_{+} \in G\left(D_{x_{0}}\right), \quad h_{\mathrm{out}} \rightarrow h_{\mathrm{out}} g_{-}, \quad g_{-} \in G\left(\Sigma_{g} \backslash x_{0}\right),
$$

отвечают действиям $G\left(D_{x_{0}}\right)$ и $G\left(\Sigma_{g} \backslash x_{0}\right)$ в представлении двойного факторпространства (3.8).

\footnotetext{
${ }^{3} \mathrm{~B}$ действительности, расслоения с $\gamma \neq 0$ нестабильны.
} 
Определим тривиальные связности на $D_{x_{0}}$ и $\Sigma_{g} \backslash x_{0}$ :

$$
\bar{A}_{\text {int }}=\bar{\partial} h_{\text {int }} h_{\text {int }}^{-1}, \quad \bar{A}_{\text {out }}=\bar{\partial} h_{\text {out }} h_{\text {out }}^{-1} .
$$

Из голоморфности $g(t)$ следует, что

$$
\bar{A}_{\text {int }}=\widehat{w} \bar{A}_{\text {out }} \widehat{w}^{-1} \text {. }
$$

Это означает, что посредством $\left(h_{\text {int }}, h_{\text {out }}\right)$ мы задаем связность $d^{\prime \prime}$ на сечениях нетривиального $G$-расслоения. Умножения справа на голоморфные преобразования (3.13) не меняют $\bar{A}_{\text {int }}$ и $\bar{A}_{\text {оut }}$. С другой стороны, умножения слева на гладкие преобразования

$$
h_{\mathrm{int}} \rightarrow f_{\mathrm{int}} h_{\mathrm{int}}, \quad f_{\mathrm{int}} \in \mathscr{G}\left(D_{x_{0}}\right), \quad h_{\mathrm{out}} \rightarrow f_{\mathrm{out}} h_{\mathrm{out}}, \quad f_{\mathrm{out}} \in \mathscr{G}\left(\Sigma_{g} \backslash x_{0}\right),
$$

такие, что

$$
f_{\text {out }}=\widehat{w} f_{\text {int }} \widehat{w}^{-1},
$$

не меняют $g(t)$, но действуют на связности калибровочными преобразованиями

$$
\bar{A}_{\text {int }} \rightarrow \bar{\partial} f_{\text {int }} f_{\text {int }}^{-1}+f_{\text {int }} \bar{A}_{\text {int }} f_{\text {int }}^{-1}, \quad \bar{A}_{\text {out }} \rightarrow \bar{\partial} f_{\text {out }} f_{\text {out }}^{-1}+f_{\text {out }} \bar{A}_{\text {out }} f_{\text {out }}^{-1} .
$$

Итак, мы определили пространство пар $\left(h_{\text {out }}, h_{\text {int }}\right)$ в разложении $(3.12)$ :

$$
\mathscr{T}\left(\Sigma_{g}, G\right)=\left\{\left(h_{\text {out }}, h_{\text {int }}\right) \in \mathscr{G}\left(D_{x_{0}}\right) \times \mathscr{G}\left(\Sigma_{g} \backslash x_{0}\right)\right\} .
$$

Существуют группы голоморфных и гладких автоморфизмов

$$
\mathscr{G}^{\text {hol }} \sim G\left(D_{x_{0}}\right) \times G\left(\Sigma_{g} \backslash x_{0}\right)
$$

И

$$
\mathscr{G}^{C^{\infty}}=\left\{\left(f_{\text {int }}, f_{\text {out }}\right): f_{\text {int }} \in \mathscr{G}\left(D_{x_{0}}\right), f_{\text {out }} \in \mathscr{G}\left(\Sigma_{g} \backslash x_{0}\right), f_{\text {out }}=\widehat{w} f_{\text {int }} \widehat{w}^{-1}\right\},
$$

действующих на $\mathscr{T}\left(\Sigma_{g}, G\right)$ по формулам (3.13) и (3.15) соответственно. Тем самым, стартуя с пространства пар $\mathscr{T}\left(\Sigma_{g}, G\right)$, получаем пространство модулей в описании Дольбо (2.7) или Дринфельда-Симпсона (3.8):

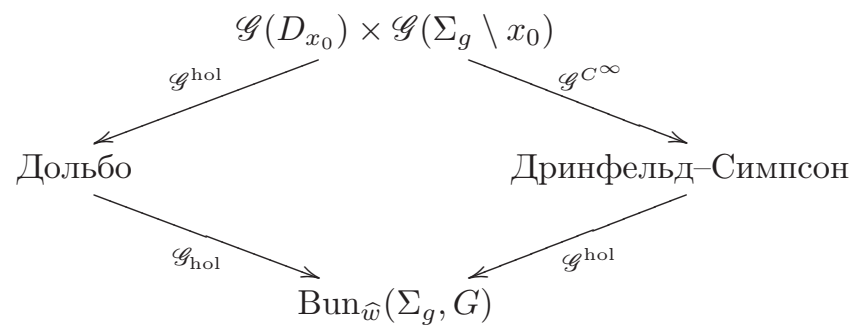

Пусть $P$ - параболическая подгруппа и $f_{\mathrm{int}}=P+O(t, \bar{t})$. Тогда приходим к описанию Дольбо для пространства модулей с qр-структурами (2.13). Эта конструкция обобщается на случай многих отмеченных точек, т. е. на $\operatorname{Bun}\left(\Sigma_{g}, \mathbf{x}, G\right)$. Предложение 3.1 доказано. 
Перейдем к более грубой шкале изучения пространства $\operatorname{Bun}\left(\Sigma_{g, n}, x_{0}, G\right)$. Рассмотрим монодромии функций переклейки $g(t) \in G\left(D^{\times}\right)$вокруг точки $x_{0}$ : $g(t \exp (2 \pi i))=\zeta g(t)$. Для компоненты, нумеруемой $\widehat{w}=w t^{\gamma}$, монодромия равна $\zeta=\exp (2 \pi i \gamma)$. Если $\gamma \in Q^{\vee}$, то монодромия тривиальна: $\zeta=1$. Если же $\gamma \in P^{\vee}$, то $\zeta=\exp (2 \pi i \gamma)$ - элемент центра $\mathscr{Z}(\bar{G})$ (А.34). Тогда, переходя к фактору $P^{\vee} / Q^{\vee} \sim \mathscr{Z}(\bar{G})$ в $(3.7)$, получаем разложение

$$
\operatorname{Bun}\left(\Sigma_{g, n}, x_{0}, G\right)=\bigcup_{\zeta \in \mathscr{Z}(\bar{G})} \operatorname{Bun}_{\zeta}\left(\Sigma_{g, n}, x_{0}, G\right) .
$$

Если $\zeta=1$, то $g(t)$ выступает в качестве функции переклейки для $\bar{G}$-расслоения $E_{\bar{G}}$. Таким образом, нетривиальные монодромии являются препятствиями для подъема $G$-расслоений до $\bar{G}$-расслоений.

Аналогичное разложение можно записать и для других факторгрупп (А.22). В случаях $A_{n-1}\left(n=p l\right.$ - составное) и $D_{n}$ центр $\mathscr{Z}(\bar{G})$ имеет нетривиальные подгруппы $\mathscr{Z}_{l} \sim \mu_{l}=\mathbb{Z} / l \mathbb{Z}$. Тогда существуют факторгруппы

$$
G_{l}=\bar{G} / \mathscr{Z}_{l}, \quad G_{p}=G_{l} / \mathscr{Z}_{p}, \quad G^{\mathrm{ad}}=G_{l} / \mathscr{Z}\left(G_{l}\right),
$$

где $\mathscr{Z}\left(G_{l}\right) \sim \mu_{p}=\mathscr{Z}(\bar{G}) / \mathscr{Z}_{l} . \quad$ Группа $\bar{G}=\operatorname{Spin}_{4 n}(\mathbb{C})$ имеет нетривиальный центр

$$
\mathscr{Z}\left(\operatorname{Spin}_{4 n}\right)=\left(\mu_{2}^{L} \times \mu_{2}^{R}\right), \quad \mu_{2}=\mathbb{Z} / 2 \mathbb{Z},
$$

а ее подгруппы описываются диаграммой (А.23). Таким образом, в общем случае следующие монодромии являются препятствиями к поднятию расслоений:

$$
\begin{aligned}
& \zeta \in \mathscr{Z}(\bar{G}) \text { - препятствия к подъему } E_{G^{\text {ad }}} \text { до } E_{\bar{G}}, \\
& \zeta \in \mathscr{Z}_{l}-\text { препятствия к подъему } E_{G_{l}} \text { до } E_{\bar{G}}, \\
& \zeta \in \mathscr{Z}\left(G_{l}\right) \text { - препятствия к подъему } E_{G^{\text {ad }}} \text { до } E_{G^{l}} .
\end{aligned}
$$

Когомологическая интерпретация препятствий возникает из трех коротких точных последовательностей:

$$
\begin{aligned}
1 & \rightarrow \mathscr{Z}(\bar{G}) \rightarrow \bar{G}\left(\mathscr{O}_{\Sigma}\right) \rightarrow G^{\mathrm{ad}}\left(\mathscr{O}_{\Sigma}\right) \rightarrow 1, \\
1 & \rightarrow \mathscr{Z}_{l} \rightarrow \bar{G}\left(\mathscr{O}_{\Sigma}\right) \rightarrow G_{l}\left(\mathscr{O}_{\Sigma}\right) \rightarrow 1 \\
1 & \rightarrow \mathscr{Z}\left(G_{l}\right) \rightarrow G_{l}\left(\mathscr{O}_{\Sigma}\right) \rightarrow G^{\mathrm{ad}}\left(\mathscr{O}_{\Sigma}\right) \rightarrow 1
\end{aligned}
$$

Из них следует длинная точная последовательность когомологий с коэффициентами в аналитических пучках:

$$
\begin{aligned}
& \rightarrow H^{1}\left(\Sigma_{g}, \bar{G}\left(\mathscr{O}_{\Sigma}\right)\right) \rightarrow H^{1}\left(\Sigma_{g}, G^{\mathrm{ad}}\left(\mathscr{O}_{\Sigma}\right)\right) \rightarrow H^{2}\left(\Sigma_{g}, \mathscr{Z}(\bar{G})\right) \sim \mathscr{Z}(\bar{G}) \rightarrow 0, \\
& \rightarrow H^{1}\left(\Sigma_{g}, \bar{G}\left(\mathscr{O}_{\Sigma}\right)\right) \rightarrow H^{1}\left(\Sigma_{g}, G_{l}\left(\mathscr{O}_{\Sigma}\right)\right) \rightarrow H^{2}\left(\Sigma_{g}, \mathscr{Z}_{l}\right) \sim \mu_{l} \rightarrow 0, \\
& \rightarrow H^{1}\left(\Sigma_{g}, G_{l}\left(\mathscr{O}_{\Sigma}\right)\right) \rightarrow H^{1}\left(\Sigma_{g}, G^{\mathrm{ad}}\left(\mathscr{O}_{\Sigma}\right)\right) \rightarrow H^{2}\left(\Sigma_{g}, \mathscr{Z}\left(G_{l}\right)\right) \sim \mu_{p} \rightarrow 0 .
\end{aligned}
$$


Первая группа когомологий $H^{1}\left(\Sigma_{g}, G\left(\mathscr{O}_{\Sigma}\right)\right)$ определяет касательное пространство к $\operatorname{Bun}\left(\Sigma_{g}, G\right)$. Элементы из $H^{2}$ - препятствия к подъему расслоений, а именно,

$$
\begin{aligned}
& H^{2}\left(\Sigma_{g}, \mathscr{Z}(\bar{G})\right) \text { - препятствия к подъему } E_{G^{\text {ad }}} \text { до } E_{\bar{G}}, \\
& H^{2}\left(\Sigma_{g}, \mathscr{Z}_{l}\right) \text { - препятствия к подъему } E_{G_{l}} \text { до } E_{\bar{G}}, \\
& H^{2}\left(\Sigma_{g}, \mathscr{Z}\left(G_{l}\right)\right) \text { - препятствия к подъему } E_{G^{\text {ad }}} \text { до } E_{G^{l}} .
\end{aligned}
$$

ОПРЕДЕЛЕНИЕ 3.1. Образы $\zeta\left(E_{G}\right)$ (как элементов группы $H^{1}\left(\Sigma_{g}, G\left(\mathscr{O}_{\Sigma}\right)\right)$ ) в $H^{2}\left(\Sigma_{g}, \mathscr{Z}\right)$ называются характеристическими классами $E_{G}$-расслоений.

Рассмотрим теперь случай многих отмеченных точек. Ассоциируем с отмеченными точками преобразования аффинной группы Вейля $W_{P}(\mathrm{~A} .14)$ :

$$
\overrightarrow{\widehat{w}}=\left(w_{1} t^{\gamma_{1}}, \ldots, w_{n} t^{\gamma_{n}}\right), \quad \gamma_{a} \in P^{\vee}
$$

Используя разложение (3.7), определим сектор в пространстве модулей (3.5)

$$
\operatorname{Bun}_{\vec{\gamma}}\left(\Sigma_{g, n}, \mathbf{x}, G^{\mathrm{ad}}\right)=G^{\mathrm{ad}}\left(\Sigma_{g} \backslash \mathbf{x}\right) G^{\mathrm{ad}}\left(D^{\times}\right) \overrightarrow{\widehat{w}} / G^{\mathrm{ad}}(D)
$$

и разложение пространства модулей

$$
\operatorname{Bun}\left(\Sigma_{g, n}, \mathbf{x}, G^{\mathrm{ad}}\right)=\bigcup_{\vec{\gamma} \in \oplus P^{\vee}} \operatorname{Bun}_{\vec{\gamma}}\left(\Sigma_{g, n}, \mathbf{x}, G^{\mathrm{ad}}\right) .
$$

В случае многих отмеченных точек сектор $\operatorname{Bun}_{\zeta}\left(\Sigma_{g, n}, \mathbf{x}, G^{\text {ad }}\right)$ как в (3.17) определяется локальными функциями переклейки $g\left(t_{a}\right)$ с монодромиями $\zeta_{a}$ вокруг точек $x_{a}$ такими, что $\zeta=\prod_{a=1}^{n} \zeta_{a} \in \mathscr{Z}(\bar{G})$. Это означает, что в (3.22) мы отождествляем компоненты $\operatorname{Bun}_{\vec{\gamma}}$ и $\operatorname{Bun}_{\vec{\gamma}^{\prime}}$, если $\sum_{a=1}^{n}\left(\gamma_{a}-\gamma_{a}^{\prime}\right) \in Q^{\vee}$. Тогда получаем разложение пространства модулей (3.17) на топологические секторы:

$$
\operatorname{Bun}\left(\Sigma_{g, n}, \mathbf{x}, G^{\mathrm{ad}}\right)=\bigcup_{\zeta \in \mathscr{Z}(\bar{G})} \operatorname{Bun}_{\zeta}\left(\Sigma_{g, n}, \mathbf{x}, G^{\mathrm{ad}}\right) .
$$

3.3. Плоские расслоения. Рассмотрим для начала модули расслоений Хиггса $T^{*} \operatorname{Bun}\left(\Sigma_{g, n}, \mathbf{x}, G^{\text {ad }}\right)$ в конструкции Дринфельда-Симпсона. Расслоения Хиггса определялись как множество пар $\left(\Phi_{a}, g_{a}\right)(a=1, \ldots, n)$, где $g_{a} \in L_{a}(G)$, а $\Phi_{a} \in \operatorname{Lie}^{*}\left(L_{a}(G)\right) \otimes d t_{a}$ - поля Хиггса. Данное многообразие является симплектическим с формой

$$
\omega=\sum_{a} \oint_{\Gamma_{a}}\left\langle\delta\left(\Phi_{a} g_{a}^{-1}\right) \wedge \delta g_{a}\right\rangle
$$

где $\Gamma_{a} \subset D_{a}^{\times}$. Действие $\mathscr{G}_{\text {in }}=G(D)$ и $\mathscr{G}_{\text {out }}=G\left(\Sigma_{g} \backslash \mathbf{x}\right)$ на эти пары поднимается на $T^{*} \operatorname{Bun}\left(\Sigma_{g, n}, \mathbf{x}, G^{\text {ad }}\right)$ :

$$
\begin{aligned}
& \mathscr{G}_{a, \text { int }}: \Phi_{a} \rightarrow f_{a, \text { int }}^{-1} \Phi_{a} f_{a, \text { int }}, \quad g \rightarrow g f_{a, \text { int }}, \\
& \mathscr{G}_{\text {out }}: \Phi_{a} \rightarrow \Phi_{a}, \quad g \rightarrow f_{\text {out }} g .
\end{aligned}
$$


Пространство модулей определяется через симплектическую редукцию

$$
\mathscr{G}_{\text {out }} \backslash \bigcup_{a=1}^{n}\left(\Phi_{a}, g_{a}\right) / / \mathscr{G}_{a, \text { int }} .
$$

Можно показать, что такое двойное симплектическое факторпространство действительно изоморфно $T^{*} \operatorname{Bun}\left(\Sigma_{g, n}, \mathbf{x}, G\right)$. Так как $\operatorname{Bun}\left(\Sigma_{g, n}, \mathbf{x}, G\right)$ является объединением секторов (3.23), то таким объединением является и

$$
T^{*} \operatorname{Bun}\left(\Sigma_{g, n}, \mathbf{x}, G\right)=\bigcup_{\vec{\gamma} \in \bigoplus P^{\vee}} T^{*} \operatorname{Bun}_{\vec{\gamma}}\left(\Sigma_{g, n}, \mathbf{x}, G^{\mathrm{ad}}\right)=\bigcup_{\zeta \in \mathscr{Z}(G)} T^{*} \operatorname{Bun}_{\zeta}\left(\Sigma_{g, n}, \mathbf{x}, G\right) .
$$

Основной интерес для нас представляют плоские расслоения. Мы докажем, чTO

$$
\operatorname{FBun}\left(\Sigma_{g, n}, \mathbf{x}, G\right)=\bigcup_{\vec{\gamma} \in \bigoplus P^{\vee}} \operatorname{FBun}_{\vec{\gamma}}\left(\Sigma_{g, n}, \mathbf{x}, G^{\mathrm{ad}}\right)=\bigcup_{\zeta \in \mathscr{Z}(G)} \operatorname{FBun}_{\zeta}\left(\Sigma_{g, n}, \mathbf{x}, G\right) .
$$

Как и выше, заменим пространство связностей $\operatorname{Conn}\left(\Sigma_{g}, G\right)(2.5)$ на множество пар $\mathscr{R}$ на $G\left(D^{\times}\right)$,

$$
\mathscr{R}=\left\{\left(\left(\partial_{t_{a}}+X_{a}\right) \otimes d t_{a}, g_{a}\right), a=1, \ldots, n\right\},
$$

где $g_{a}$ принадлежит $G\left(D_{a}^{\times}\right)(3.1)$ и может иметь нетривиальную монодромию, a $\left.X_{a} \in \mathfrak{g}\left(D_{a}^{\times}\right)=\mathfrak{g} \otimes \mathbb{C}\left[t_{a}^{-1}, t_{a}\right]\right]\left(\right.$ А.58). Компонента $\left(\partial_{t_{a}}+X_{a}\right) \otimes d t_{a}$ принадлежит главному однородному пространству $P H / T^{*} G\left(D_{a}^{\times}\right)$над кокасательным расслоением $T^{*} G\left(D_{a}^{\times}\right)$.

Форма $\omega(2.6)$ в этой параметризации переписывается в следующем виде (cм. (3.24)):

$$
\omega=\sum_{a} \oint_{\Gamma_{a}}\left\langle\delta\left(X_{a} g_{a}^{-1}\right) \wedge \delta g_{a}\right\rangle+\frac{1}{2} \oint_{\Gamma_{a}}\left\langle g_{a}^{-1} \delta g_{a} \wedge \partial_{t}\left(g^{-1} \delta g_{a}\right)\right\rangle
$$

ЗАмЕчАниЕ 3.1. В действительности пары $\left(\left(\partial_{t_{a}}+X_{a}\right) \otimes d t_{a}, g_{a}\right)$ следует заменить на $\left(\left(\kappa_{a} \partial_{t_{a}}+X_{a}\right) \otimes d t_{a},\left(g_{a}, \lambda_{a}\right)\right)$, где $\lambda_{a}$ - центральное расширение группы петель $G\left(D_{a}^{\times}\right)$, а $\kappa_{a} \in \mathbb{C}$ - двойственные, коцентральные расширения [71]. В этом случае $\omega$ приобретает дополнительный член $\sum_{a} \delta \kappa_{a} \wedge \delta \log \lambda_{a}$. Можно доказать, что эти скалярные поля не входят в уравнения изомонодромных деформаций. По этой причине рассматривать коцентральные расширения $\lambda_{a}$ не будем. Тем не менее мы сохраним параметр $\kappa$, так как в пределе $\kappa \rightarrow 0$ (в п. 4.5) получим таким способом интегрируемые системы.

Симплектическая форма (3.29) является инвариантной относительно калибровочных преобразований из $\mathscr{G}_{a, \text { int }}=L_{a}^{+}(G)(3.4)$ и $\mathscr{G}_{\text {out }}=G\left(\Sigma_{g} \backslash \mathbf{x}\right)$ :

$$
\begin{aligned}
\mathscr{G}_{a, \text { int }}: X_{a} \rightarrow \operatorname{Ad}_{f_{a, \text { int }}}^{*}\left(X_{a}\right)= & f_{a, \text { int }}^{-1} \partial_{t_{a}} f_{a, \text { int }} \\
& +f_{a, \text { int }}^{-1} X_{a} f_{a, \text { int }}, \quad g_{a} \rightarrow g_{a} f_{a, \text { int }},
\end{aligned}
$$

$$
\mathscr{G}_{\text {out }}: X_{a} \rightarrow X_{a}, \quad g_{a} \rightarrow f_{\text {out }} g \text {. }
$$


Они генерируются гамильтонианами

$$
F_{a, \text { int }}=\oint_{\Gamma_{a}}\left\langle\varepsilon_{a, \text { int }}, X_{a}\right\rangle, \quad F_{\text {out }}=\sum_{a} \oint_{\Gamma_{a}}\left\langle\varepsilon_{\text {out }},\left(g X_{a} g^{-1}-\partial_{t_{a}} g g^{-1}\right)\right\rangle .
$$

Здесь $\varepsilon_{\text {out }} \in \operatorname{Lie}\left(G\left(\Sigma_{g} \backslash \mathbf{x}\right)\right)$ и

$$
\varepsilon_{a, \text { int }} \in \operatorname{Lie}\left(L_{a}^{+}(G)\right), \quad \varepsilon_{a, \text { int }}=x_{a, 0}+t_{a} x_{a, 1}+\cdots, \quad x_{a, 0} \in \mathfrak{p}_{a}=\operatorname{Lie}\left(P_{a}\right) .
$$

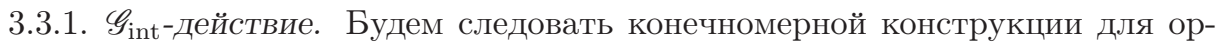
бит коприсоединенного действия (А.47)-(А.55). Отображение момента, отвечающее $\mathscr{G}_{a, \mathrm{int}}$-действию, равно $\mu_{\mathrm{int}}=\sum_{a} \mu_{a}, \mu_{a}=\left.\operatorname{Pr}\right|_{\operatorname{Lie}^{*}\left(L_{a}^{+} G\right)}\left(X_{a}\right)$, где двойственное пространство определяется как

$$
\operatorname{Lie}^{*}\left(L_{a}^{+} G\right)=\left\{y_{a, 0} t_{a}^{-1}+y_{a, 1} t_{a}^{-2}+\cdots, y_{a, 0} \in \mathfrak{p}_{a}^{*}\right\} .
$$

Из (А.36) и (А.63) находим, что $\mathfrak{p}_{a}^{*}=\mathfrak{g}_{a}^{\prime} \oplus \widetilde{\mathfrak{h}}_{a}^{*} \oplus \mathfrak{n}_{a}^{-}$. Положим уровень момента равным

$$
\mu_{a}=\left.\operatorname{Pr}\right|_{\operatorname{Lie}^{*}\left(L_{a}^{+} G\right)}\left(X_{a}\right)=\nu_{a} t_{a}^{-1}, \quad \nu_{a} \in \mathfrak{s}_{a}^{*} \quad\left(\mathfrak{s}_{a}^{*}-\right.\text { подалгебра Леви (А.36)). }
$$

Иными словами, поле $A$ имеет простые полюсы

$$
X_{a}\left(t_{a}\right)=\left(\nu_{a}+\xi_{a,-1}\right) t_{a}^{-1}+\xi_{a, 0}+O\left(t_{a}\right)=\nu_{a} t_{a}^{-1}+\xi\left(t_{a}\right), \quad \xi_{a,-1} \in \mathfrak{n}^{+}
$$

Это значит, что $\xi\left(t_{a}\right)$ - произвольный элемент пространства $\mathfrak{b}\left(t_{a}\right)=\mathfrak{n}^{+} t_{a}^{-1}+$ $\left.\mathfrak{g} \otimes \mathbb{C}\left[t_{a}\right]\right]$. Заметим, что $\mathscr{G}_{a, \text { int }}$ сохраняет $\nu_{a} t_{a}^{-1}$ и для $\nu_{a} \neq 0$ действует свободно на $\mathfrak{b}\left(t_{a}\right)$ :

$$
\operatorname{Ad}_{L_{a}^{+} G}^{*} X_{a}\left(t_{a}\right)=\nu_{a} t_{a}^{-1}+\xi^{\prime}\left(t_{a}\right), \quad \xi^{\prime}\left(t_{a}\right)=\xi_{a,-1}^{\prime} t_{a}^{-1}+\xi_{a, 0}^{\prime}+O\left(t_{a}\right) .
$$

Из (3.30) и (3.34) находим, что симплектическое факторпространство задается следующим множеством пар:

$$
\left(g_{a}\left(t_{a}\right), \nu_{a} t_{a}^{-1}+\xi\left(t_{a}\right)\right) \quad(a=1, \ldots, n)
$$

с отношением эквивалентности

$$
\left(g_{a}\left(t_{a}\right), \nu_{a} t_{a}^{-1}+\xi\left(t_{a}\right)\right) \sim\left(g_{a}\left(t_{a}\right) f\left(t_{a}\right), \operatorname{Ad}_{f\left(t_{a}\right)}^{*}\left(\nu_{a} t_{a}^{-1}+\xi\left(t_{a}\right)\right)\right), \quad f\left(t_{a}\right) \in G\left(D_{a}\right) .
$$

Зафиксируем калибровку $G\left(D_{a}\right)$-действия в $(3.35)$, положив $\xi\left(t_{a}\right)=0$. Подгруппа Леви $L_{a} \subset \mathscr{G}_{a \text {,int }}($ А.38) сохраняет такую калибровку:

$$
L_{a}=\left\{f\left(t_{a}\right) \in G\left(D_{a}^{\times}\right) \mid\left(\operatorname{Ad}^{*}\right)_{f\left(t_{a}\right)}^{-1} \nu_{a}=\nu_{a}\right\}
$$


Из (3.35) следует, что $g_{a}\left(t_{a}\right)$ определяется с точностью до умножения справа на $L_{a}$. Следовательно, редуцированное пространство в этом случае является коприсоединенной орбитой:

$$
\begin{aligned}
\mathscr{O}_{a}^{\mathrm{aff}} & =G\left(D_{a}^{\times}\right) / L_{a}=\left\{g\left(t_{a}\right) \in G\left(D_{a}^{\times}\right) \mid X_{a}\left(t_{a}\right)=\left(\mathrm{Ad}^{*}\right)_{g\left(t_{a}\right)}^{-1} \nu_{a} t_{a}^{-1}\right\} \\
& \left.=-\partial_{t_{a}} g\left(t_{a}\right) g^{-1}\left(t_{a}\right)+g\left(t_{a}\right) \nu_{a} t_{a}^{-1} g^{-1}\left(t_{a}\right)\right\} .
\end{aligned}
$$

Это пространство является главным однородным пространством над кокасательными расслоениями на многообразиях аффинных флагов $P H / T^{*} \mathrm{Flag}_{a}^{\text {aff }}$ $\left(\operatorname{Flag}_{a}^{\text {aff }} \sim G\left(D_{a}^{\times}\right) / G\left(D_{a}\right)\right)$. Форма $(3.29)$ на $\mathscr{O}_{p_{a}}$ принимает вид

$$
\omega_{\mathscr{O}_{a}^{\text {aff }}}=\oint_{\Gamma_{a}}\left\langle\delta\left(\nu_{a} t_{a}^{-1} g_{a}^{-1}\right) \wedge \delta g_{a}\right\rangle+\frac{1}{2} \oint_{\Gamma_{a}}\left\langle\left(g_{a}^{-1} \delta g_{a}\right) \wedge \partial_{t_{a}}\left(g^{-1} \delta g_{a}\right)\right\rangle .
$$

Таким способом, посредством гамильтоновой редукции по $\mathscr{G}_{\text {int }}=\prod_{a} \mathscr{G}_{a, \text { int }}$ получаем из $\mathscr{R}(3.28)$ пространство

$$
\bigcup_{a=1}^{n} \mathscr{O}_{a}^{\mathrm{aff}}=\mathscr{R} \| \prod_{a} \mathscr{G}_{a, \mathrm{int}} .
$$

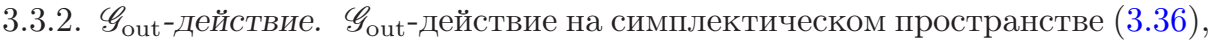
(3.37) производит момент

$$
\mu_{\text {out }}=\left.\operatorname{Pr}\right|_{\operatorname{Lie}^{*}\left(G\left(\Sigma_{g} \backslash x_{0}\right)\right)} X(t)=0, \quad X(t)=g \frac{\nu}{t} g^{-1}-\partial_{t} g g^{-1} .
$$

Пусть $G(t, z)$ - ядро Коши на проколотой кривой $\Sigma_{g} \backslash x_{0}$. Оно является функцией по первому аргументу и 1-формой по второму. Оно регулярно вне диагонали, на которой имеет полюс первого порядка. Его существование следует из аффинности проколотой кривой.

ПРЕДЛОЖЕНИЕ 3.2. Пустъ $\Gamma_{0}-$ малый контур вокруг $x_{0}$. Форма

$$
\widetilde{A}(z)=\operatorname{Res}_{t=0}(X(t) G(t, z)),
$$

где $X(t)$ удовлетворяет $(3.38)$, голоморфна на $\Sigma_{g} \backslash \Gamma_{0}$. Ее асимптотика в пределе $z \rightarrow \Gamma_{0}$ coвnадает с $X(t)$.

ДокАЗАТЕЛЬСТво. Вычислим $k$-й коэффициент разложения Лорана формы $\widetilde{A}$. Он равен

$$
\begin{aligned}
\frac{1}{2 \pi i} \oint_{\Gamma_{0}} s^{-k-1} \widetilde{A}(s) & =\frac{1}{2 \pi i} \oint_{\Gamma_{0}} s^{-k-1}\left(\operatorname{Res}_{t=0}(X(t) G(t, s))\right) \\
& =\operatorname{Res}_{t=0}\left(X(t) \frac{1}{2 \pi i} \oint s^{-k-1} G(t, s)\right) .
\end{aligned}
$$

Изменение порядка интегрирования и взятия вычета корректно. Вычет берется в нуле по $t$, т. е. интеграл вычисляется при условии $|t|<|s|$. Подынтегральное выражение имеет особенности при $s=t$ и $s=0$. Вклад от первой равен $t^{-k-1}$, а от второй $\left.(1 / k !)\left(\partial_{s}\right)^{k} G(t, s)\right|_{s=0}$. Вычет произведения $\mu_{\text {out }}$ с первым членом суммы дает $k$-й коэффициент $\mu_{\text {out }}$, а вычет произведения со вторым обращается в нуль, так как выражение $(1 / k !) \partial^{k} G(p, s) /\left.\partial s^{k}\right|_{s=0}$ регулярно вне проколов. Предложение доказано. 
Определим на $\Sigma_{g} \backslash x_{0}$ голоморфную связность

$$
A_{\text {out }}=h_{\text {out }}(\partial+\widetilde{A}) h_{\text {out }}^{-1}=h_{\text {out }} \widetilde{A} h_{\text {out }}^{-1}-\partial_{z} h_{\text {out }} h_{\text {out }}^{-1},
$$

где $h_{\text {оut }}$ определяется из разложения $(3.12)$. На $D_{x_{0}}^{\times}$

$$
A_{\text {out }}=h_{\text {out }} X(t) h_{\text {out }}^{-1}-\partial_{t} h_{\text {out }} h_{\text {out }}^{-1},
$$

где $X(t)$ определено в (3.38). Из (3.14) и предложения 3.2 следует, что на $\Sigma_{g} \backslash x_{0}$ кривизна обращается в нуль:

$$
F\left(A_{\text {out }}, \bar{A}_{\text {out }}\right)=\left[\partial+A_{\text {out }}, \bar{\partial}+\bar{A}_{\text {out }}\right]=h_{\text {out }}[\partial+\widetilde{A}, \bar{\partial}] h_{\text {out }}^{-1}=0 .
$$

Определим $A_{\text {int }}$ формулой (см. (3.14) и (3.38))

$$
A_{\text {int }}=h_{\text {int }} \nu t^{-1} h_{\text {int }}^{-1}-\partial_{t} h_{\text {int }} h_{\text {int }}^{-1} .
$$

Из (3.40) следует, что

$$
A_{\mathrm{out}}=\widehat{w} A_{\mathrm{int}} \widehat{w}^{-1} .
$$

Кривизна на $D_{x_{0}}$ принимает вид

$$
F\left(A_{\text {int }}, \bar{A}_{\text {int }}\right)=\left(h_{\text {int }} \nu h_{\text {int }}^{-1}\right) \bar{\partial} t^{-1} .
$$

Tак как $h_{\text {int }}(t, \bar{t})=h_{0}+O(t, \bar{t})$, получаем

$$
F\left(A_{\text {int }}, \bar{A}_{\text {int }}\right)=\mathbf{S} \delta(t), \quad \mathbf{S}=h_{0} \nu h_{0}^{-1} .
$$

Таким образом, стартуя с $\operatorname{Conn}\left(\Sigma_{g}, G\right)=\mathscr{R}(3.28)$, получаем плоские расслоения с фуксовой особенностью.

3.4. Преобразования Гекке. Преобразование Гекке - это сингулярное калибровочное преобразование, “сплетающее" сечения расслоений, отвечающих различным компонентам в смысле элементов Вейля в (3.22) или элементов центра в (3.23).

Во избежание дополнительных усложнений рассмотрим случай одной отмеченной точки $\Sigma_{g} \backslash x_{0}$. Голоморфное расслоение $E_{G}$ задается в описании Дринфельда-Симпсона (3.22) или (3.23) функцией переклейки $g(t)$. Заменим ее на $g(t) h(t)$, где $h(t) \in L(G)$ может иметь нетривиальную монодромию вокруг $t=0$. Вследствие $(3.5), h(t)$ определена с точностью до умножения справа на $f(t) \in L^{+}(G)$. Как объяснялось в п. 3.2, это преобразование изменяет характеристический класс $E_{G}$. Из (А.68) следует, что в двойном факторпространстве можно выбрать представителя $h(t)=\widehat{w} \in W_{t(G)}$, т. е.

$$
g(t) \rightarrow g(t) \widehat{w}, \quad \widehat{w}=w t^{\gamma}, \quad t^{\gamma}=\mathbf{e}(\log (t \gamma)), \quad \gamma \in t(G),
$$

где $t(G)$ - решетка ковесов (А.31). Монодромия $t^{\gamma}$ есть $\exp (-2 \pi i \gamma)$. Так как $\langle\alpha, \gamma\rangle \in \mathbb{Z}$, для любого $\mathbf{x} \in \mathfrak{g}$ получаем $\operatorname{Ad}_{\exp (-2 \pi i \gamma)} \mathbf{x}=\mathbf{x}$. Тогда $\exp (-2 \pi i \gamma)$ есть элемент $\mathscr{Z}(\bar{G})\left(\right.$ А.34). Если матрица перехода $g(t)$, задающая $E_{G}$, имеет 
тривиальную монодромию, то новая матрица (3.42) приобретает нетривиальную монодромию. Таким образом, получаем новое расслоение $\widetilde{E}_{G}$ с нетривиальным характеристическим классом $\zeta\left(\widetilde{E}_{G}\right)$. Расслоение $\widetilde{E}_{G}$ называется модифицированным. Еще одно название модификации - преобразование Гекке. Если $\gamma \in Q^{\vee}$, то $\zeta=1$ и $\widetilde{E}_{G}$ имеет тот же характеристический класс, что и $E_{G}$. Как следует из (А.68) и (3.6), пространство преобразований Гекке типа $\gamma$ совпадает с клеткой Шуберта $C_{\widehat{w}}(\mathrm{~A} .71)$. Его размерность равна $l(\widehat{w})(\mathrm{A} .72)$.

Для преобразования сечений используем обозначение

$$
\Xi(\zeta): \Gamma\left(E_{G}\right)=\Gamma(E(\zeta=1)) \rightarrow \Gamma\left(\widetilde{E}_{G}\right)=\Gamma(E(\zeta))
$$

Если $d+\mathscr{A}-$ связность на $E_{G}$, а $d+\widetilde{\mathscr{A}}-$ на $\widetilde{E}_{G}$, то

$$
(d+\widetilde{\mathscr{A}}) \Xi(\zeta)=\Xi(\zeta)(d+\mathscr{A}) .
$$

Сформулируем ограничения на выбор $\gamma \in P^{\vee}$ для расслоений с qр-структурой в $x_{0}$. Предположим, что модификация сохраняет qр-структуру в отмеченной точке $x_{0}$. В этом случае $\widehat{w}$ является нормализатором $G(D)=L^{+}(G)$ в разложении (3.7):

$$
\widehat{w}^{-1} L^{+}(G) \widehat{w}=L^{+}(G) .
$$

Напомним, что $\gamma$ задает параболическую подалгебру $\mathfrak{p}_{\gamma} \subset \mathfrak{g}($ А.43), а тем самым и qр-структуру.

ПредЛОЖЕНИЕ 3.3. Пустъ $\gamma \in \widehat{\Upsilon}^{\vee}$ - допустимый фундаментальный ковес (А.45), а $\mathfrak{p}_{\gamma}=\mathfrak{s}+\mathfrak{n}^{+}$- допустимая относительно $\gamma$ параболическая подалгебра (А.44) в разложении $\operatorname{Lie}\left(L^{+}(G)\right)=\mathfrak{p}+t \mathfrak{g}+o(t)$. Если

$$
\operatorname{Ad}_{w} \mathfrak{n}^{+}=\mathfrak{n}^{-}
$$

то $\widehat{w}$ нормализует $L^{+}(G)$.

ДоказАтельство. Пусть $x(t) \in \operatorname{Lie}\left(L^{+}(G)\right), x(t)=a+b+t\left(a_{1}+b_{1}+c_{1}\right)+o(t)$, где $a, a_{1} \in \mathfrak{s}, b, b_{1} \in \mathfrak{n}^{+}$и $c_{1} \in \mathfrak{n}^{-}$. Тогда вследствие (А.44)

$$
\operatorname{Ad}_{\widehat{w}}=\operatorname{Ad}_{w}\left(\left(a+c_{1}\right)+t a_{1}+t^{2} b_{1}+\cdots\right) .
$$

Если $\operatorname{Ad}_{w}^{*} \mathfrak{n}^{+}=\mathfrak{n}^{-}$, то $\operatorname{Ad}_{w}\left(a+c_{1}\right) \in \mathfrak{p}=\mathfrak{s}+\mathfrak{n}^{+}$и $\operatorname{Ad}_{w} b_{1} \in \mathfrak{n}^{-}$, т. е. qр-структура восстановлена. Предложение доказано.

Для общих максимальных параболических подгрупп такие преобразования не существуют. Для классических групп оно существует для $\operatorname{SL}(2 n, \mathbb{C})$ и подгруппы Леви $\mathrm{S}(\mathrm{GL}(n, \mathbb{C}) \times \mathrm{GL}(n, \mathbb{C}))$, а также в случаях $B, C$ и $D$ для $\gamma=\varpi_{1}^{\vee}$ (см. табл. 4 в приложении В). Тем не менее, если $P$ не является максимальной $\left(P \subset P_{\gamma}\right)$, то преобразование Гекке может сохранять qр-структуру в большем числе случаев. Например, если $P=B$ - подгруппа Бореля (3.42) $w: \alpha \rightarrow-\alpha$, то получаем (3.45). 
Преобразования Гекке голоморфных расслоений поднимаются до преобразований Гекке плоских расслоений. В этом случае вместо многообразий флагов отмеченным точкам приписаны орбиты, определяемые по подалгебрам Леви $\mathfrak{s}\left(\Pi^{\prime}\right)$ (А.36). По этой причине вместо (3.46) имеем

$$
\operatorname{Ad}_{\widehat{w}} \mathfrak{s}=\widetilde{\mathfrak{s}} .
$$

Здесь $\widehat{w}=w t^{\gamma}$ и $\gamma \in \widehat{\Upsilon}^{\vee}$. Тогда $\widetilde{\mathfrak{s}}=\operatorname{Ad}_{w} \mathfrak{s}$. Хотя это преобразование и изменяет подгруппу Леви $L \rightarrow \widetilde{L}$, оно сохраняет орбиту $\mathscr{O}=G / L$. Следовательно, для плоских расслоений требуется только $\gamma \in \widehat{\Upsilon}^{\vee}$.

\section{4. Гамильтонов подход к изомонодромной задаче}

4.1. Деформация комплексных структур на кривых. Комплексная структура на $\Sigma_{g}$ определяется оператором $\bar{\partial}$. Рассмотрим его деформацию под действием замены переменных

$$
w=z-\varepsilon(z, \bar{z}), \quad \bar{w}=\bar{z}-\overline{\varepsilon(z, \bar{z})},
$$

где $\varepsilon(z, \bar{z})$ - малая вариация. С точностью до общего множителя частные производные приобретают вид

$$
\left\{\begin{array}{l}
\partial_{w}=\partial_{z}+\bar{\mu} \partial_{\bar{z}}, \\
\partial_{\bar{w}}=\partial_{\bar{z}}+\mu \partial_{z},
\end{array}\right.
$$

где

$$
\mu=\frac{\bar{\partial} \varepsilon}{1-\partial \varepsilon} \sim \bar{\partial} \varepsilon
$$

дифференциал Бельтрами, $\mu \in \Omega^{(-1,1)}\left(\Sigma_{g}\right)$. Перейдем от локальных координат $(w, \bar{w})(4.1)$ к киральным $(w, \widetilde{w})$ :

$$
w=z-\varepsilon(z, \bar{z}), \quad \widetilde{w}=\bar{z},
$$

так как $\bar{\mu}$ в нашей конструкции несущественен. Пара $(w, \widetilde{w})$ также является парой локальных координат на $\Sigma_{g}$. В них частные производные приобретают вид

$$
\left\{\begin{array}{l}
\partial_{w}=\partial_{z}, \\
\partial_{\widetilde{w}}=\partial_{\bar{z}}+\mu \partial_{z} .
\end{array}\right.
$$

Важно, что $\partial_{\widetilde{w}}$ зануляет голоморфные функции: $\partial_{\widetilde{w}} f(w)=0$. Гладкая функция $w(z, \bar{z}) \in C^{\infty}\left(\Sigma_{g}\right)$ задает глобальный диффеоморфизм кривой $\Sigma_{g}$. Будем говорить, что дифференциал Бельтрами $\mu(z, \bar{z})$ эквивалентен $\mu^{\prime}(z, \bar{z})$, если $\mu^{\prime}(z, \bar{z})=\mu(w(z, \bar{z}), \bar{w}(z, \bar{z}))$. Отношение эквивалентности в $\Omega^{(-1,1)}\left(\Sigma_{g}\right)$ под действием $\operatorname{Diff}_{C} \infty\left(\Sigma_{g}\right)$ - это пространство модулей $\mathfrak{M}\left(\Sigma_{g, n}\right)$ комплексных структур на $\Sigma_{g}$. Касательным к нему является пространство Тейхмюллера $\mathfrak{T}_{g} \sim$ $H^{1}\left(\Sigma_{g}, \Gamma\right)$, где $\Gamma \in T \Sigma_{g}$. По теореме Римана-Роха имеем

$$
\operatorname{dim}\left(\mathfrak{T}_{g}\right)=3(g-1)
$$


Пусть $\left(\mu_{1}^{0}, \ldots, \mu_{l}^{0}\right)$ - базис в векторном пространстве $H^{1}\left(\Sigma_{g}, \Gamma\right)$. Тогда

$$
\mu=\sum_{l=1}^{3 g-3} \tau_{l} \mu_{l}^{0}
$$

где локальные координаты $\tau_{l}$ будут играть роль времен в задаче изомонодромной деформации.

Подвижные отмеченные точки. Рассмотрим пространство модулей $\mathfrak{M}\left(\Sigma_{g, n}\right)$ комплексных структур кривых с отмеченными точками $\Sigma_{g, n}$. Это пространство расслаивается над пространством модулей комплексных структур компактных кривых $\mathfrak{M}\left(\Sigma_{g}\right)$ со слоями $\mathscr{U} \subset \mathbb{C}^{n}$, отвечающими подвижным отмеченным точкам.

Пространство $\mathfrak{M}\left(\Sigma_{g, n}\right)$ - классы смежности отношения эквивалентности в пространстве дифференциалов $\Omega^{(-1,1)}\left(\Sigma_{g, n}\right)$ по действию группы диффеоморфизмов $\operatorname{Diff}_{C \infty}\left(\Sigma_{g, n}\right)$, зануляющихся в отмеченных точках.

Рассмотрим локальные координаты в слое. Пусть $(z, \bar{z})$ - локальные координаты в окрестности отмеченных точек $x_{a}^{0}(a=1, \ldots, n)$, а $\mathscr{U}_{a}$ - сами окрестности точек $x_{a}^{0}$ такие, что $\mathscr{U}_{b} \cap \mathscr{U}_{a}=\varnothing$. Зададим $C^{\infty}$-функцию

$$
\chi_{a}(z, \bar{z})= \begin{cases}1, & z \in \mathscr{U}_{a}^{\prime} \subset \mathscr{U}_{a}, \\ 0, & z \notin \mathscr{U}_{a} .\end{cases}
$$

Подвижные точки $\left(x_{a}^{0} \rightarrow x_{a}\right)$ отвечают следующим локальным деформациям координат (4.1):

$$
\begin{aligned}
w=z-\sum_{a=1}^{n} \varepsilon_{a}(z, \bar{z}), \quad \varepsilon_{a}(z, \bar{z}) & =-t_{a} \chi_{a}(z, \bar{z})+\sum_{j>0} t_{a}^{(j)}\left(z-x_{a}^{0}\right)^{j} \chi_{a}(z, \bar{z}), \\
t_{a} & =x_{a}-x_{a}^{0}
\end{aligned}
$$

Действие $\operatorname{Diff}_{C^{\infty}}\left(\Sigma_{g, n}\right)$ позволяет положить $t_{a}^{(j)}=0$ для $j>0$. Таким образом, в общем случае имеем только $n$ времен $t_{a}$. Часть дифференциала Бельтрами, связанная с отмеченными точками, приобретает вид

$$
\mu=\sum_{a=1}^{n} t_{a} \mu_{a}^{(0)}, \quad \mu_{a}^{(0)}=\bar{\partial} \chi_{a}(z, \bar{z})
$$

В итоге локальные координаты на $\mathfrak{T}\left(\Sigma_{g, n}\right)$ есть множество

$$
\left\{\left(\tau_{1}, \ldots, \tau_{3(g-1)}, t_{1}, \ldots, t_{n}\right)\right\}
$$

и

$$
\operatorname{dim}\left(\mathfrak{T}\left(\Sigma_{g, n}\right)\right)=3(g-1)+n
$$


4.2. Уравнения движения и изомонодромная задача. Зафиксируем комплексную структуру на $\Sigma_{g, n}$ и заменим связности (2.5) парами

$$
\left(\left(\kappa \partial_{z}+A\right) \otimes d z,\left(\bar{\partial}+\bar{A}^{\prime}\right) \otimes d \bar{z}\right) .
$$

Здесь, следуя П. Делиню, введем понятие $\kappa$-связностей, где $\kappa$ - малый параметр (в некотором смысле напоминающий постоянную Планка). Пространство модулей $\kappa$-связностей было исследовано в [72]. Сами $\kappa$-связности позволяют перейти в квазиклассическом пределе к полям Хиггса $\Phi=\lim _{\kappa \rightarrow 0}\left(\kappa \partial_{z}+A\right) \otimes d z \in$ $\Omega^{0}\left(\Sigma_{g}\right.$, Lie $\left.G \otimes K\right)$, где $K$ - канонический класс на $\Sigma_{g}$. В этом пределе уравнения изомонодромных деформаций переходят в уравнения движения интегрируемых систем типа Хитчина. Соответствующая линейная задача становится изоспектральной вместо изомонодромной до взятия предела. Эту процедуру опишем ниже более детально.

Рассмотрим поляризацию (4.3) в деформированных координатах:

$$
\left(\kappa \partial_{w}+A\right) \otimes d w, \quad\left(\partial_{\widetilde{w}}+\bar{A}\right) \otimes d \widetilde{w} .
$$

Компонента связности $\bar{A}^{\prime}$ в этих координатах определяется следующим обра30м:

$$
\bar{A}^{\prime}=\bar{A}-\frac{1}{\kappa} \mu A, \quad(\bar{\partial}+\mu \partial+\bar{A}) \otimes d \widetilde{w}=\left(\partial_{\bar{w}}+\bar{A}\right) \otimes d \widetilde{w} .
$$

Форма $\omega(2.6)$ имеет вид

$$
\omega=\int_{\Sigma_{g}}(\delta A \wedge \delta \bar{A})-\frac{1}{\kappa} \int_{\Sigma_{g}}(A, \delta A) \delta \mu
$$

Форму $\omega$ можно рассматривать как дифференциал от 1-формы Пуанкаре-Картана $\left(\omega=\omega^{\mathrm{PC}}=\delta \vartheta^{\mathrm{PC}}\right)[73]$ на расширенном пространстве $(A, \bar{A}, \mu)$. В канонических координатах на фазовом пространстве $\left(p_{j}, q_{j}\right)$ и для гамильтонианов $H_{l}\left(\vec{p}, \vec{q} ; t_{1}, \ldots, t_{n}\right)$ она принимает вид

$$
\vartheta^{\mathrm{PC}}=\sum_{j} p_{j} \delta q_{j}-\sum_{l} H_{l}\left(\vec{p}, \vec{q} ; t_{1}, \ldots, t_{n}\right) \delta t_{l} .
$$

Связности $(A, \bar{A})$ играют роль канонических переменных на $\operatorname{Conn}\left(\Sigma_{g}, G\right)$, а второй член описывает спаривание дифференциалов квадратичных гамильтонианов с отвечающими им временами. Более точно, учитывая (4.6), запишем $\omega$ KaK

$$
\omega^{\mathrm{PC}}=\omega_{0}-\frac{1}{\kappa} \sum_{l=1}^{3 g-3} \delta H_{l} \delta \tau_{l}, \quad H_{l}=\frac{1}{2} \int_{\Sigma_{g}}(A, A) \mu_{l}^{0}, \quad \omega_{0}=\int_{\Sigma_{g}}(\delta A \wedge \delta \bar{A}) .
$$

Форма Пуанкаре-Картана позволяет записать функционал действия

$$
S=\sum_{l=1}^{3 g-3} \int_{0}^{\infty}\left(\int_{\Sigma_{g}}\left(A, \partial_{l} \bar{A}\right)-\frac{1}{2 \kappa}(A, A) \mu_{l}^{0}\right) d \tau_{l} \quad\left(\partial_{l}=\partial_{\tau_{l}}\right)
$$


Уравнения движения, следующие из этого действия (либо из гамильтонианов), имеют вид

$$
\partial_{l} \bar{A}=\frac{1}{\kappa} A \mu_{l}^{0}, \quad \partial_{l} A=0 .
$$

Они являются условиями совместности для линейной системы

$$
\begin{aligned}
& \left(\kappa \partial_{w}+A\right) \psi=0, \\
& \left(\partial_{\bar{w}}+\bar{A}\right) \psi=0, \\
& \kappa \partial_{\tau_{l}} \psi=0,
\end{aligned}
$$

где $\partial_{\bar{w}}=\bar{\partial}+\mu \partial$, а $\psi \in \Omega^{(0)}\left(\Sigma_{g}\right.$, Aut $\left.E_{G}\right)$. Уравнения движения (4.14) на $A$ и $\bar{A}$ - это условия совместности (4.15a) $\&(4.15 \mathrm{c})$ и $(4.15 \mathrm{~b}) \&(4.15 \mathrm{c})$. Монодромия $\psi$ - преобразование

$$
\psi \rightarrow \psi \mathscr{Y}, \quad \mathscr{Y} \in \operatorname{Rep}\left(\pi_{1}\left(\Sigma_{g}\right) \rightarrow G\right) .
$$

Уравнение (4.15c) означает, что монодромия не зависит от времен. Совместность $(4.15 \mathrm{a})$ и $(4.15 \mathrm{~b})$ - это условие $(2.2)$. Форма $\omega^{\mathrm{PC}}$ задана на расслоении $\mathscr{P}(G)$ над пространством Тейхмюллера $\mathfrak{T}_{g}$. Его слои - пространство гладких связностей $\operatorname{Conn}\left(\Sigma_{g}, G\right)$ :

$$
\begin{aligned}
& \mathscr{P}(G) \\
& \downarrow \downarrow \operatorname{Conn}\left(\Sigma_{g}, G\right) \\
& \mathfrak{T}_{g}
\end{aligned}
$$

Линейные уравнения (4.14) описывают свободное движение на $\operatorname{Conn}\left(\Sigma_{g}, G\right)$. Они становятся нетривиальными на $\operatorname{FBun}\left(\Sigma_{g}, G\right)$.

4.3. Вклад от отмеченных точек. Предположим, что $(1,0)$-компонента связности, как и в (2.17), имеет простые полюсы в отмеченных точках:

$$
\operatorname{Res}_{z=x_{a}} A=\mathbf{S}^{a}, \quad \overrightarrow{\mathbf{S}}=\left(\mathbf{S}^{1}, \ldots, \mathbf{S}^{n}\right) .
$$

Расслоение $\mathscr{P}(G)$ задано теперь над пространством Тейхмюллера $\mathfrak{T}_{g, n}$. Множество его слоев - гладкие связности $\operatorname{Conn}\left(\Sigma_{g, n}, G\right)$ с особенностями в отмеченных точках. Следовательно, локальными координатами на $\mathscr{P}(G)$ является набор переменных

$$
(A, \bar{A}, \overrightarrow{\mathbf{S}}, \mathbf{t}), \quad \mathbf{t}=\left(\tau_{1}, \ldots, \tau_{3 g-3} ; t_{1}, \ldots, t_{n}\right) .
$$

Расслоение $\mathscr{P}(G)$ играет роль расширенного фазового пространства, в то время как $\operatorname{Conn}\left(\Sigma_{g, n}, G\right)$ - обычное фазовое пространство, а $\mathbf{t}$ - набор времен. Форма (4.13) приобретает дополнительные члены:

$$
\begin{gathered}
\omega^{\mathrm{PC}}=\omega^{\mathrm{Conn}}-\frac{1}{\kappa}\left(\sum_{l=1}^{3 g-3} \delta H_{l} \delta \tau_{l}+\sum_{a=1}^{n} \delta H_{a} \delta t_{a}\right), \quad \omega^{\mathrm{Conn}}=\omega_{0}+\sum_{a=1}^{n} \omega_{a}^{\mathrm{KK}}, \\
H_{a}=\frac{1}{2} \int_{\mathscr{U}_{a}}(A, A) \bar{\partial} \chi_{a}(z, \bar{z}) .
\end{gathered}
$$


Эта форма генерирует $(3 g-3+n)$ векторных полей $D_{s}$ таких, что $\omega\left(D_{s}, \cdot\right)=0$, где

$$
\begin{array}{rlrl}
D_{l} & =\partial_{\tau_{l}}+\frac{1}{\kappa}\left\{H_{l}, \cdot\right\}_{\omega_{0}} & & (l=1, \ldots, 3 g-3), \\
D_{a}=\partial_{t_{a}}+\frac{1}{\kappa}\left\{H_{a}, \cdot\right\}_{\omega_{a}} & (a=1, \ldots, n) .
\end{array}
$$

Скобки Пуассона, отвечающие форме $\omega_{0},-$ это скобки Дарбу, а форме $\omega_{a}^{\mathrm{KK}}-$ это скобки Пуассона-Ли. Последние скобки невырождены на слоях. Векторные поля $D_{s}$ задают уравнения движения для любой функции $f$ на $\mathscr{P}(G)$ :

$$
\frac{d f}{d r_{s}}=\partial_{r_{s}} f+\frac{1}{\kappa}\left\{H_{s}, f\right\}, \quad r_{s}=\tau_{s} \quad \text { или } \quad r_{s}=t_{s} .
$$

Уравнения (4.14) являются их частным случаем. Условия совместности - это так называемые уравнения Уизема [74]:

$$
\kappa \partial_{s} H_{r}-\kappa \partial_{r} H_{s}+\left\{H_{r}, H_{s}\right\}=0 .
$$

В рациональном случае $\tau$-функции исследовались в [9]. Для уравнений Пенлеве I-VI они изучались в [75].

Гамильтонианы коммутируют относительно скобки Пуассона и представляют собой квадратичные гамильтонианы Хитчина. Это означает, что существует производящая функция (тау-функция)

$$
H_{s}=\frac{\partial}{\partial t_{s}} \log \tau, \quad \tau=\exp \left(\frac{1}{2} \sum_{s=1}^{l} \int_{\Sigma_{g, n}}\left\langle A^{2}\right\rangle \mu_{s}\right) .
$$

4.4. Симплектическая редукция. До сих пор уравнения движения, линейная задача и тау-функция были тривиальными. Содержательные уравнения возникают в результате наложения связей (2.18) и фиксации калибровки. Форма $\omega^{\mathrm{PC}}(4.17)$ инвариантна относительно действия калибровочной группы $\mathscr{G}_{\mathrm{P}}(2.12)$ :

$$
A \rightarrow f^{-1} \kappa \partial f+f^{-1} A f, \quad \bar{A} \rightarrow f^{-1} \bar{\partial} f+f^{-1} \bar{A} f .
$$

Зафиксируем $\bar{A}$ таким образом, что $\bar{L}$ параметризует общие орбиты $\mathscr{G}_{\mathrm{B}}$-действия:

$$
\bar{A}=f(\bar{\partial}+\mu \partial) f^{-1}+f \bar{L} f^{-1} .
$$

Тогда поле $L$, двойственное $\bar{L}$, получается из $A$ действием того же элемента $f$, определенного с точностью до умножения слева на элементы, сохраняющие $\bar{L}$ :

$$
L=f^{-1} \kappa \partial f+f^{-1} A f .
$$

Таким образом, в локальных координатах уравнение момента принимает следующий вид (см. (2.18)):

$$
(\bar{\partial}+\partial \mu) L-\kappa \partial \bar{L}+[\bar{L}, L]=2 \pi i \sum_{a=1}^{n} \mathbf{S}^{a} \delta\left(x_{a}\right) .
$$


Условие фиксации калибровки (4.21) и условие для отображения момента задают редуцированное пространство $\operatorname{FBun}\left(\Sigma_{g, n}, G\right)=\{L, \bar{L}, \mathbf{S}\}$. Оно становится конечномерным (см. (2.23)), равно как и расслоение $\mathscr{P}(G)$ :

$$
\begin{aligned}
\operatorname{dim}(\mathscr{P}(G)) & =\operatorname{dim}\left(\mathrm{FBun}\left(\Sigma_{g, n}, \mathbf{x}, G\right)\right)+\operatorname{dim}\left(\mathfrak{T}_{g, n}\right) \\
& =2 \operatorname{dim}(G)(g-1)+\sum_{a=1}^{n} \operatorname{dim}\left(\mathscr{O}_{a}\right)+3 g-3+n .
\end{aligned}
$$

В силу инвариантности $\omega(4.17)$, форма на $\operatorname{FBun}\left(\Sigma_{g, n}, \mathbf{x}, G\right)$ имеет вид

$$
\begin{array}{cc}
\omega=\omega^{\mathrm{FBun}}-\frac{1}{\kappa} \sum_{s=1}^{3 g-3+n} \delta H_{s} \delta \tau_{s}, \quad H_{s}(L)=\frac{1}{2} \int_{\Sigma_{g, n}}\left\langle L^{2}\right\rangle \mu_{s}^{0}, \\
\omega^{\mathrm{FBun}}=\omega_{0}+\sum_{a=1}^{n} \omega_{a}^{\mathrm{KK}}, & \omega_{0}=\int_{\Sigma_{g, n}}\langle d L, d \bar{L}\rangle .
\end{array}
$$

Вследствие (4.23) система более не является свободной, так как $L$ зависит от модулей плоских расслоений и $\mathbf{S}$. Более того, так как $H_{s}$ явно зависит от времен, редуцированная система является неавтономной.

Пусть $M_{s}=\partial_{s} f f^{-1}$. Как следует из (4.21) и (4.22), уравнения движения (4.14) на пространстве $\operatorname{FBun}\left(\Sigma_{g, n}, \mathbf{x}, G\right)$ имеют вид

$$
\begin{aligned}
& \kappa \partial_{s} L-\kappa \partial M_{s}+\left[M_{s}, L\right]=0 \quad(s=1, \ldots, l), \\
& (\bar{\partial}+\partial \mu) M_{s}=-L \mu_{s}^{0} .
\end{aligned}
$$

Уравнения (4.26a) - уравнения Лакса. Существенная разница с интегрируемыми системами состоит в дополнительном члене $\kappa \partial M_{s}$. $\operatorname{Ha} \operatorname{FBun}\left(\Sigma_{g, n}, G\right)$ линейная система (4.15) принимает вид

$$
\begin{aligned}
& (\kappa \partial+L) \psi=0, \\
& \left(\partial_{\bar{w}}+\bar{L}\right) \psi=0, \\
& \left(\kappa \partial_{s}+M_{s}\right) \psi=0 \quad\left(s=1, \ldots, l_{2}\right) .
\end{aligned}
$$

Уравнения (4.26a) и (4.26b) являются условиями совместности для линейных задач (4.27a)\&(4.27c) и (4.27b)\&(4.27c), а (4.27a)\&(4.27b) - это условие (4.23). Уравнения (4.27c) обеспечивают свойство изомонодромности системы (4.27a), $(4.27 \mathrm{~b})$ относительно вариаций времен $t_{s}$. Нелинейные уравнения (4.26) будем называть иерархией изомонодромных деформаций.

Преобразования Гекке действуют на сечениях $\psi(3.43): \widetilde{\psi}=\Xi(\zeta) \psi$. В результате от исходной системы (4.27) приходим к модифицированной:

$$
\begin{aligned}
& (\kappa \partial+\widetilde{L}) \widetilde{\psi}=0 \\
& \left(\partial_{\bar{w}}+\widetilde{\bar{L}}\right) \widetilde{\psi}=0 \\
& \left(\kappa \partial_{s}+\widetilde{M}_{s}\right) \widetilde{\psi}=0 \quad\left(s=1, \ldots, l_{2}\right) .
\end{aligned}
$$


Преобразование

$$
\Xi(\zeta):\left(L, M_{s}\right) \rightarrow\left(\widetilde{L}, \widetilde{M}_{s}\right)
$$

можно рассматривать как дискретную динамику. В этом смысле операторы Гекке задают преобразования Бэклунда уравнений изомонодромных деформаций.

4.5. Изомонодромные деформации и интегрируемые системы. Как отмечалось выше, изомонодромные уравнения можно интерпретировать как деформацию (квантование Уизема) интегрируемых уравнений. Параметром деформации является $\kappa$. Для систем Шлезингера такой подход был развит в [76].

Определим независимые времена $t_{s}^{0}=\left(\tau_{l}^{0}, x_{a}^{0}\right)$ как $\tau_{l}=\tau_{l}^{0}+\kappa t_{l}, t_{a}=\kappa \widetilde{t}_{a}$ для $\kappa \rightarrow 0$. Это означает, что $t_{s}=\left(t_{l}, \tilde{t}_{a}\right)$ играют роль локальных координат в окрестности точки $\left(t_{l}^{0}, x_{a}^{0}\right) \in \mathfrak{T}_{g, n}$. В этом пределе уравнения движения (4.26a) - это стандартные уравнения Лакса

$$
\partial_{s} L^{(0)}+\left[M_{s}^{(0)}, L^{(0)}\right]=0 \quad(s=1, \ldots, l),
$$

где $L^{(0)}=L\left(t_{s}^{0}\right)\left(M_{s}^{(0)}=M_{s}\left(t_{s}^{0}\right)\right)$. Линейная задача для этих систем получается из линейной задачи для изомонодромной задачи (4.27) аналогично квазиклассическому пределу в квантовой механике. Представим функцию Бейкера-Ахиезера в форме ВКБ-приближения

$$
\psi=\Phi \exp \left(\frac{\mathscr{S}^{(0)}}{\kappa}+\mathscr{S}^{(1)}\right)
$$

и подставим (4.30) в линейную систему (4.27). Если $\partial_{\bar{z}} \mathscr{S}^{(0)}=0$ и $\partial_{\tau_{l}^{0}} \mathscr{S}^{(0)}=$ $\partial_{x_{a}^{0}} \mathscr{S}^{(0)}=0$, то члены порядка $\kappa^{-1}$ исчезают. В квазиклассическом пределе положим $\partial \mathscr{S}^{(0)}=\lambda$. В нулевом порядке приближения получим систему:

$$
\begin{aligned}
& \left(\lambda+L^{(0)}\left(z, \tau_{0}\right)\right) Y=0, \\
& \partial_{\bar{z}} Y=0, \\
& \left(\partial_{t_{s}}+M_{s}^{(0)}\left(z, \tau_{0}\right)\right) Y=0 .
\end{aligned}
$$

Функция Бейкера-Ахиезера $Y$ принимает вид

$$
Y=\Phi \exp \left(\sum_{s} t_{s} \frac{\partial}{\partial t_{s}^{0}} \mathscr{S}^{(0)}\right)
$$

Условие совместности (4.31a) и (4.31b) - уравнение Лакса (4.29).

\section{Глава II. Изомонодромные задачи на эллиптических кривых}

\section{5. Модули плоских расслоений на эллиптических кривых}

Пространство модулей плоских $G$-расслоений играет роль фазового пространства изомонодромной задачи. Для тривиальных расслоений это пространство было описано в [77]-[79], а для нетривиальных - в [80], [81]. 
5.1. Голоморфные расслоения на эллиптических кривых. Здесь и далее будем рассматривать пространства модулей голоморфных $\operatorname{Bun}\left(\Sigma_{\tau}, \mathbf{x}, G\right)$ и плоских $\operatorname{FBun}\left(\Sigma_{\tau}, \mathbf{x}, G\right)$ расслоений на эллиптических кривых, реализованных в виде $\Sigma_{\tau} \sim \mathbb{C} /(\tau \mathbb{C} \oplus \mathbb{C})(\operatorname{Im} \tau>0)$. Два генератора $\rho_{1}$ и $\rho_{\tau}$ фундаментальной группы $\pi_{1}\left(\Sigma_{\tau}\right)$ отвечают сдвигам $z \rightarrow z+1$ и $z \rightarrow z+\tau$ и удовлетворяют соотношению

$$
\rho_{1} \rho_{\tau} \rho_{1}^{-1} \rho_{\tau}^{-1}=1 .
$$

Сечения $G$-расслоения $E_{G}(V)$ над $\Sigma_{\tau}$ удовлетворяют условиям квазипериодичности:

$$
\psi(z+1)=\mathscr{Q} \psi(z), \quad \psi(z+\tau)=\Lambda \psi(z),
$$

где функции переклейки $(\mathscr{Q}, \Lambda)$ в свою очередь удовлетворяют (5.1):

$$
\mathscr{Q}(z) \Lambda^{-1}(z) \mathscr{Q}^{-1}(z+\tau) \Lambda(z+1)=1 .
$$

По определению эти функции перехода задают топологически тривиальное расслоение. Пусть $\zeta$ - элемент из $\mathscr{Z}(G)$. Для получения нетривиального расслоения заменим (5.3) на уравнение

$$
\mathscr{Q}(z) \Lambda^{-1}(z) \mathscr{Q}^{-1}(z+\tau) \Lambda(z+1)=\zeta .
$$

Из (А.33) следует, что правая часть представляется в виде $\zeta=\mathbf{e}(\gamma)(\mathbf{e}(x)=$ $\exp (2 \pi i x))$ с $\gamma \in P^{\vee}$ (А.10). Тогда (5.4) принимает вид

$$
\mathscr{Q}(z) \Lambda^{-1}(z) \mathscr{Q}^{-1}(z+\tau) \Lambda(z+1)=\mathbf{e}(\gamma) .
$$

Расслоение $\widetilde{E}$ эквивалентно $E$, когда его сечения $\widetilde{s} \psi$ связаны с $\psi$ следующим образом: $\widetilde{\psi}(z)=f(z) \psi(z)$, где $f(z)$ - обратимый оператор на $V$. Из (5.5) видим, что преобразованные операторы перехода приобретают вид

$$
\mathscr{Q}^{f}=f(z+1) \mathscr{Q} f^{-1}(z), \quad \Lambda^{f}=f(z+\tau) \Lambda f^{-1}(z) .
$$

Такие преобразования образуют группу автоморфизмов $\mathscr{G}$ (калибровочную группу) расслоения $E_{G}$ над $\Sigma_{\tau}$. Из [82] известно, что операторы перехода (они же функции переклейки) можно выбрать постоянными. Следовательно,

$$
\left[\mathscr{Q}, \Lambda^{-1}\right]=\mathbf{e}(\gamma) \quad\left(\left[\mathscr{Q}, \Lambda^{-1}\right]=\mathscr{Q} \Lambda^{-1} \mathscr{Q}^{-1} \Lambda\right) .
$$

Таким образом, $(\mathscr{Q}, \Lambda)$ образуют проективное представление $\pi_{1}\left(\Sigma_{\tau}\right)$. Пространство модулей стабильных голоморфных расслоений над $\Sigma_{\tau}$ определяется следующим образом:

$$
\operatorname{Bun}_{\zeta}\left(\Sigma_{\tau}, G\right)=\left\{\left[\mathscr{Q}, \Lambda^{-1}\right]=\zeta\right\} / \mathscr{G}, \quad \zeta \in \mathscr{Z}(G) .
$$

В [77]-[79] было показано, что $\operatorname{Bun}_{\zeta}\left(\Sigma_{\tau}, G\right)$ для $\zeta=1$ изоморфно взвешенному проективному пространству. Пусть $G=\bar{G}$ - односвязная группа. Зафиксируем подгруппу Картана $\mathscr{H}_{\bar{G}} \subset \bar{G}$. Предположим, что элемент $\mathscr{Q}$ является полупростым и, следовательно, сопряжен общему элементу из $\mathscr{H}_{\bar{G}}$. Исключая неполупростые функции переклейки, мы определяем в пространстве модулей большую клетку $\operatorname{Bun}^{0}\left(\Sigma_{\tau}, G\right)$, т. е. заменяем (5.8) на

$$
\operatorname{Bun}_{\zeta}^{0}\left(\Sigma_{\tau}, G\right)=\left\{\left[\mathscr{Q}, \Lambda^{-1}\right]=\zeta\right\} / \mathscr{G}, \quad \mathscr{Q} \in \mathscr{H}_{\bar{G}} .
$$


ПреДЛОЖЕНИЕ 5.1. Решения (5.9) имеют следующее описание. ${ }^{4}$

- Элемент $\Lambda$ имеет вид $\Lambda=\Lambda^{0} \mathbf{e}(\mathbf{u})$, где $\Lambda^{0}$ определяется однозначно по ковесу $\gamma\left(\Lambda^{0}=\Lambda_{\gamma}^{0}\right)$. Это специальный элемент из группы Вейля $W$, сохраняющий расширенную систему корней $\Pi^{\vee \text { ехt }}=\Pi^{\vee} \cup \alpha_{0}^{\vee} u$, таким образом, являющийся симметрией расширенной диаграммы Дынкина.

•• Пусть $\widetilde{\mathfrak{h}}_{0} \subseteq \mathfrak{h}-$ подалгебра Картана, сохраняемая элементом $\Lambda^{0}\left(\lambda\left(\widetilde{\mathfrak{h}}_{0}\right)=\right.$ $\left.\widetilde{\mathfrak{h}}_{0}, \lambda=\operatorname{Ad}_{\Lambda^{0}}\right)$. Тогдa $\mathbf{u} \in \widetilde{\mathfrak{h}}_{0}$.

•• Элемент $\mathscr{Q}$ имеет вид $\mathscr{Q}=\mathscr{Q}^{0}$, где

$$
\mathscr{Q}^{0}=\exp (2 \pi i \varrho U), \quad \varrho=\frac{\rho^{\vee}}{h} \in \mathfrak{H},
$$

$h$ - число Кокстера, $\rho^{\vee}=\frac{1}{2} \sum_{\alpha^{\vee} \in\left(R^{\vee}\right)^{+}} \alpha^{\vee} u U$ коммутируют с $\Lambda^{0} .5$

ЗАмечание 5.1. Для $\operatorname{Spin}(4 n)$ центр $\mathscr{Z}(\operatorname{Spin}(4 n)) \sim \mu_{2} \times \mu_{2}$ имеет два генератора $\zeta_{1}$ и $\zeta_{2}$, отвечающих фундаментальным весам $\varpi_{a}, \varpi_{b}$ левого и правого спинорных представлений. Действуя по вышеуказанному рецепту, получим два решения $\Lambda_{a}$ и $\Lambda_{b}$ для $(5.9)$, а $\mathscr{Q}$ - одинаковый в обоих случаях.

ЗАмечАниЕ 5.2. Если $\xi \in Q^{\vee}$, то $\zeta=\mathrm{Id}$. Тогда преобразование $\Lambda_{0}$ тривиально $\Lambda_{0}=\operatorname{Id}$ и $\widetilde{\mathfrak{h}}_{0}=\mathfrak{h}$. В этом случае характеристический класс у расслоения тривиален, но существуют голоморфные модули, объединенные в вектор $\mathbf{u} \in \mathfrak{h}$.

5.2. Разложение алгебр Ли. Рассмотрим элемент $\zeta \in \mathscr{Z}(\bar{G})$ порядка $l$ и отвечающий ему $\Lambda^{0} \in W$ из предложения 5.1. Тогда $\Lambda^{0}$ генерирует циклическую группу $\mu_{l}=\left(\Lambda^{0},\left(\Lambda^{0}\right)^{2}, \ldots,\left(\Lambda^{0}\right)^{l}=1\right)$, изоморфную подгруппе $\mathscr{Z}_{l} \subseteq \mathscr{Z}(\bar{G})$. Заметим, что $l$ - делитель $\operatorname{ord}(\mathscr{Z}(\bar{G}))$. Рассмотрим действие $\Lambda^{0}$ на $\mathfrak{g}$. Так как $\left(\Lambda^{0}\right)^{l}=\mathrm{Id}$, имеем $l$-периодическую градуировку

$$
\begin{gathered}
\mathfrak{g}=\bigoplus_{j=0}^{l-1} \mathfrak{g}_{j}, \quad \lambda\left(\mathfrak{g}_{j}\right)=\omega^{j} \mathfrak{g}_{j}, \quad \omega=\exp \left(\frac{2 \pi i}{l}\right), \quad \lambda=\operatorname{Ad}_{\Lambda^{0}}, \\
{\left[\mathfrak{g}_{j}, \mathfrak{g}_{k}\right]=\mathfrak{g}_{j+k} \quad(\bmod l),}
\end{gathered}
$$

где $\mathfrak{g}_{0}$ - подалгебра $\mathfrak{g}$, а подпространства $\mathfrak{g}_{a}$ - ее представления. Более того, в этих терминах форма Киллинга на $\mathfrak{g}$ приобретает вид

$$
(\mathfrak{g}, \mathfrak{g})=\sum_{j=0}^{l-1}\left(\mathfrak{g}_{j}, \mathfrak{g}_{l-j}\right) .
$$

$\lambda$-инвариантная подалгебра $\mathfrak{g}_{0}$ содержит простую подалгебру $\widetilde{\mathfrak{g}}_{0}$ :

$$
\mathfrak{g}_{0}=\widetilde{\mathfrak{g}}_{0} \oplus V .
$$

Компоненты этого разложения ортогональны относительно формы Киллинга (В.14):

$$
\left(\mathfrak{g}_{0}, \mathfrak{g}_{0}\right)=\left(\widetilde{\mathfrak{g}}_{0}, \widetilde{\mathfrak{g}}_{0}\right)+(V, V),
$$

\footnotetext{
${ }^{4}$ См. доказательство в [45].

${ }^{5}$ Данное утверждение содержится в [83] (предложение 5 в п. VI.3.2).
} 
a $V$ - представление $\widetilde{\mathfrak{g}}_{0}$. Ниже мы явно находим $\mathfrak{g}_{0}$ для всех простых алгебр Ли из нашего списка. Система кокорней П в $\widetilde{\mathfrak{g}}_{0}$ строится усреднением по орбитам $\lambda$-действия на $\Pi^{\vee}$ ext $[45]$. Суммируем информацию об инвариантных подалгебрах в табл. 4 из приложения В.

5.3. Пространство модулей голоморфных расслоений. Мы описываем $G$-расслоения $E_{G}(V)$ операторами перехода $\Lambda=\Lambda^{0} \mathbf{e}(\mathbf{p}), \mathscr{Q}=\mathbf{e}\left(\rho^{\vee} / h+\mathbf{q}\right)$ с $\Lambda^{0}=\Lambda^{0}(\gamma)$, отвечающим ковесу $\gamma \in P^{\vee}$. Топологический тип $E$ определяется элементом $\zeta=\mathbf{e}\left(\varpi^{\vee}\right) \in P^{\vee} / t(G)$.

Преобразуем $(\Lambda, \mathscr{Q})$ при помощи $(5.6)$ с $f=\mathbf{e}(-\mathbf{q} z)$. Так как $f$ коммутирует с $\Lambda^{0}$, получим новые операторы перехода $\mathscr{Q}=\mathbf{e}(\varrho+\mathbf{q}) \rightarrow \mathscr{Q}=\mathbf{e}(\varrho), \Lambda \rightarrow$ $\Lambda^{0} \mathbf{e}(\mathbf{p}-\mathbf{q} \tau)$. Обозначим $\mathbf{p}-\mathbf{q} \tau=\mathbf{u}$. Сечения $E_{G}(V)$ обладают свойствами квазипериодичности:

$$
\psi(z+1)=\pi(\mathbf{e}(\varrho)) \psi(z), \quad \psi(z+\tau)=\pi\left(\mathbf{e}(\widetilde{\mathbf{u}}) \Lambda^{0}\right) \psi(z) .
$$

Тогда получаем

$$
\mathscr{Q}=\mathbf{e}(\varrho), \quad \Lambda=\mathbf{e}(\mathbf{u}) \Lambda^{0} .
$$

Здесь $\mathbf{u} \in \widetilde{\mathfrak{h}}_{0}$ играет роль параметра на пространстве модулей $\widetilde{\mathfrak{h}}_{0}$ (предложение 5.1). В этом разделе мы опишем его детально. На самом деле $\widetilde{\mathfrak{h}}_{0}$ покрывает истинное пространство модулей, которое и опишем.

Фиксация $\mathscr{Q}$ и $\Lambda$ все еще оставляет часть калибровочной симметрии, которая сохраняет $\widetilde{\mathfrak{h}}_{0}$. Пусть $\widetilde{W}_{0}-$ группа Вейля алгебры Ли $\widetilde{\mathfrak{g}}_{0}$, a $t\left(\widetilde{G}_{0}\right)-$ группа кохарактеров $\left(\right.$ А.31), (А.31) инвариантной подгруппы $\widetilde{G}_{0}(\mathrm{~A} .29): t\left(\widetilde{G}_{0}\right)=$ $\left\{\mathbb{C}^{*} \rightarrow \widetilde{\mathscr{H}}_{0}\right\}=\left\{\chi_{\gamma}(z)=\mathbf{e}(\gamma z)\right\}$. Кохарактеры обладают свойствами квазипериодичности:

$$
\chi_{\gamma}(z+1)=\chi_{\gamma}(z), \quad \chi_{\gamma}(z+\tau)=\chi_{\gamma}(\tau) \chi_{\gamma}(z), \quad \gamma \in t\left(\widetilde{G}_{0}\right) .
$$

Пусть $\mathscr{G}_{0}=\{f(z)\}-$ группа отображений $\Sigma_{\tau} \rightarrow \widetilde{\mathscr{H}_{0}}$,

$$
\widetilde{\mathscr{G}}_{0}=\left\{f(z)=\sum_{\gamma \in \mathscr{R}^{\vee}} c_{\gamma} \chi_{\gamma}(z)\right\},
$$

с $c_{\gamma}=0$ почти для всех $\gamma$ и

$$
\widetilde{\mathscr{R}}^{\vee}= \begin{cases}\widetilde{Q}^{\vee}, & \widetilde{G}_{0}=\widetilde{\widetilde{G}}_{0}, \\ \widetilde{P}^{\vee}, & \widetilde{G}_{0}=\widetilde{G}_{0}^{\text {ad }}, \\ \widetilde{t}\left(G_{l}\right), & \widetilde{G}_{0}=\widetilde{G}_{0, l},\end{cases}
$$

Из (5.18) следует, что $f(z+1)=f(z)$. Группа остаточных калибровочных преобразований является полупрямым произведением:

$$
\mathscr{G}_{\widetilde{\mathscr{H}}_{0}}=\widetilde{W}_{0} \ltimes \widetilde{\mathscr{G}}_{0}
$$

Преобразования сечений с помощью $f(z)=\chi_{\gamma}(z)$ записывается в виде

$$
\psi(z) \rightarrow \mathbf{e}(\gamma z) \psi(z), \quad \gamma \in \widetilde{\mathscr{R}}^{\vee}
$$


Для операторов перехода находим (см. (5.6)):

$$
\mathscr{Q}^{f}=\mathbf{e}(\gamma z) \mathscr{Q} \mathbf{e}(-\gamma z)=\mathscr{Q}, \quad \Lambda_{0}^{f}=\mathbf{e}(\gamma \tau) \mathbf{e}(\gamma z) \Lambda_{0} \mathbf{e}(-\gamma z)=\mathbf{e}(\gamma \tau) \Lambda_{0} .
$$

Так как $\gamma \tau \in \widetilde{\mathfrak{h}}_{0}$, то действие $\mathscr{G}_{\widetilde{\mathscr{H}_{0}}}$ на $\mathbf{u}$ принимает вид

$$
\mathbf{u} \rightarrow \mathbf{u}^{f}= \begin{cases}s \mathbf{u}, & s \in \widetilde{W}_{0}, \\ \mathbf{u}+\tau \gamma_{1}+\gamma_{2}, & \gamma=\left(\gamma_{1}, \gamma_{2}\right) \in \widetilde{\mathscr{R}}^{\vee}\end{cases}
$$

Таким образом, операторы перехода, задаваемые параметрами $\mathbf{u}$ либо $\mathbf{u}^{f}$, описывают эквивалентные расслоения. Полупрямое произведение группы Вейля $\widetilde{W}_{0}$ и решетки $\tau \widetilde{\mathscr{R}}^{\vee} \oplus \widetilde{\mathscr{R}}^{\vee}$ называется группой Бернштейна-Швари,мана [77]-[79]. Это эллиптический аналог аффинной группы Вейля:

$$
W_{\mathrm{BS}}\left(\mathscr{R}^{\vee}\right)=\widetilde{W}_{0} \ltimes\left(\tau \widetilde{\mathscr{R}}^{\vee} \oplus \widetilde{\mathscr{R}}^{\vee}\right) .
$$

Тем самым, u можно выбрать из фундаментальной области $C\left(\mathscr{R}^{\vee}\right)$ группы $W_{\mathrm{BS}}\left(\mathscr{R}^{\vee}\right)$ :

$$
\begin{array}{r}
\operatorname{Bun}_{\zeta}^{0}\left(\Sigma_{\tau}, G\right)=\widetilde{\mathfrak{h}}_{0} / W_{\mathrm{BS}}=C\left(\mathscr{R}^{\vee}\right)-\underset{\text { пространство модулей }}{ } \\
\text { голоморфных } \bar{G} \text {-расслоений. }
\end{array}
$$

Пусть $\mathscr{Z}(\bar{G})$ - циклическая группа порядка $N$, а $\mathscr{Z}_{l} \subseteq \mathscr{Z}(\bar{G})$ - ее подгруппа порядка $l$. Это как раз случай $\bar{G}=\mathrm{SL}(N, \mathbb{C})$ или $\operatorname{Spin}_{2 n+2}$. Для $\bar{G}-, G_{l^{-}}, G_{p^{-}}$, $G^{\mathrm{ad}}$-расслоений мы имеем следующий набор соотношений между пространствами модулей:

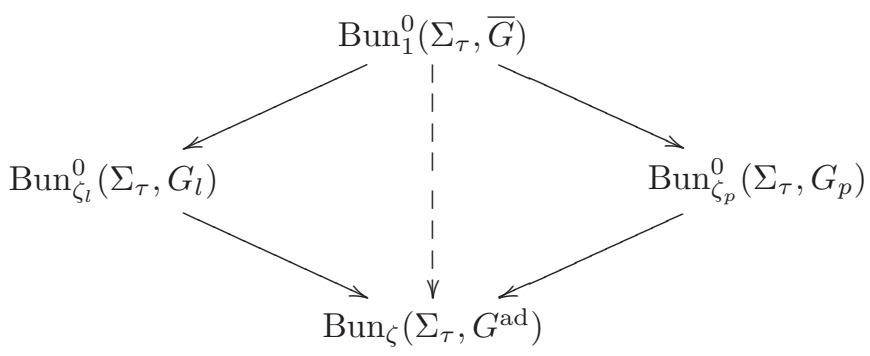

Стрелки означают накрытия. Заметим, что $\operatorname{Bun}_{1}^{0}\left(\Sigma_{\tau}, \bar{G}\right), \operatorname{Bun}_{\zeta}\left(\Sigma_{\tau}, G^{\text {ad }}\right)$, а также $\operatorname{Bun}_{\zeta_{l}}^{0}\left(\Sigma_{\tau}, G_{l}\right), \operatorname{Bun}_{\zeta_{p}}^{0}\left(\Sigma_{\tau}, G_{p}\right)$ двойственны друг другу в том смысле, что двойственны задающие их решетки. Аналогичная картина имеет место и для группы $\operatorname{Spin}_{4 n}$, для которой $\mathscr{Z}\left(\operatorname{Spin}_{4 n}\right) \sim\left(\mu_{2} \oplus \mu_{2}\right)$.

5.3.1. Голоморфные расслоения с квазипараболическими структурами. Как и в общем случае, отмеченным точкам $\mathbf{x}=\left\{x_{a}\right\}$ припишем $G$-флаги Flag $a$ $G / P_{a}\left(P_{a}-\right.$ параболическая подгруппа в $\left.G\right)$. Таким образом мы расширяем пространство $\widetilde{\mathfrak{h}}_{0}$ :

$$
\widetilde{\mathfrak{h}}_{0} \times \bigcup_{a=1}^{n} \operatorname{Flag}_{a} .
$$


Учитывая действие $\mathscr{G}_{\widetilde{\mathscr{H}_{0}}}$, находим, что большая клетка $\operatorname{Bun}_{\zeta}^{0}\left(\Sigma_{\tau}, \mathbf{x}, G\right)$ на пространстве модулей $\operatorname{Bun}_{\zeta}\left(\Sigma_{\tau}, \mathbf{x}, G\right)$ голоморфных расслоений с квазипараболическими структурами есть фактор

$$
\operatorname{Bun}_{\zeta}^{0}\left(\Sigma_{\tau}, \mathbf{x}, G\right)=\left(\widetilde{\mathfrak{h}}_{0} \times \bigcup_{a=1}^{n} \operatorname{Flag}_{a}\right) / \mathscr{G}_{\widetilde{\mathscr{H}_{0}}},
$$

где действие $\mathscr{G}_{\widetilde{\mathscr{H}}_{0}}$ на $\mathbf{Y}_{a} \in \operatorname{Flag}_{a}$ имеет вид

$$
\mathbf{Y}_{a} \rightarrow \begin{cases}s\left(\mathbf{Y}_{a}\right), & s \in \widetilde{W}_{0}, \\ \operatorname{Ad}_{\chi_{\gamma}\left(x_{a}\right)}\left(\mathbf{Y}_{a}\right), & \gamma \in \widetilde{\mathscr{R}}^{\vee}\end{cases}
$$

Тем самым мы параметризуем $\operatorname{Bun}_{\zeta}^{0}\left(\Sigma_{\tau}, \mathbf{x}, G\right)$ :

$$
\operatorname{Bun}_{\zeta}^{0}\left(\Sigma_{\tau}, \mathbf{x}, G\right)=\left\{\mathbf{u} \in C\left(\mathscr{R}^{\vee}\right) ; \mathbf{Y}_{a} \in \operatorname{Flag}_{a} / \mathscr{G}_{\widetilde{\mathscr{H}_{0}}}, a=1, \ldots, n\right\} .
$$

5.3.2. Плоские расслоения с квазипараболическими структурами. Как и в общем случае (2.9), пространство модулей плоских расслоений $\operatorname{FBun}\left(\Sigma_{\tau}, \mathbf{x}, G\right)$ над эллиптической кривой $\Sigma_{\tau}$ будем описывать как главные однородные пространства $P T^{*} \operatorname{Bun}_{\zeta}^{0}\left(\Sigma_{\tau}, \mathbf{x}, G\right)$ над кокасательными расслоениями $T^{*} \operatorname{Bun}_{\zeta}^{0}\left(\Sigma_{\tau}, \mathbf{x}, G\right)$. Пусть $\mathbf{v}-$ вектор в слоях проекции

$$
\pi: \operatorname{FBun}_{\zeta}^{0}\left(\Sigma_{\tau}, G\right) \stackrel{\text { Flat }}{\longrightarrow} \operatorname{Bun}_{\zeta}^{0}\left(\Sigma_{\tau}, G\right) .
$$

Вектор v, дуальный $\mathbf{u}$, определяется оператором перехода $\Lambda(\mathbf{u})(5.17)$. В этих терминах форма $\omega_{0}(4.25)$ принимает вид $(\delta \mathbf{v} \wedge \delta \mathbf{u})$.

Напомним, что коприсоединенная орбита $\mathscr{O}$ есть главное однородное пространство $\mathscr{O}=P T^{*}$ Flag. Таким образом, приходим к пространству

$$
\widetilde{\operatorname{FBun}}_{\zeta}^{0}\left(\Sigma_{\tau}, \mathbf{x}, G\right)=\left\{(\mathbf{v}, \mathbf{u}) ; \mathbf{S}^{a} \in \mathscr{O}_{a}, a=1, \ldots, n\right\} .
$$

$\widetilde{\operatorname{FBun}}_{\zeta}^{0}\left(\Sigma_{\tau}, \mathbf{x}, G\right)$ - симплектическое многообразие с формой (4.25):

$$
\omega=(\delta \mathbf{v} \wedge \delta \mathbf{u})+\sum_{a=1}^{n} \omega_{a}^{\mathrm{KK}} .
$$

Оказывается, эта форма зануляется векторными полями, которые генерируются $\widetilde{\mathscr{G}}_{0}$ (5.19). Таким образом описывается структура пространства модулей плоских расслоений. Пространство модулей плоских расслоений над эллиптическими кривыми с квазипараболической структурой есть симплектическое факторпространство:

$$
\operatorname{FBun}_{\zeta}^{0}\left(\Sigma_{\tau}, \mathbf{x}, G\right)=\widetilde{\operatorname{FBun}}_{\zeta}^{0}\left(\Sigma_{\tau}, \mathbf{x}, G\right) / / \tilde{\mathscr{G}}_{0}=\left\{(\mathbf{v}, \mathbf{u}) ; \mathbf{S}^{a} \in \mathscr{O}_{a} / / \tilde{\mathscr{G}}_{0}\right\} .
$$

Тогда $\omega(5.31)$ принимает вид

$$
\omega^{\mathrm{FBun}}=(\delta \mathbf{v} \wedge \delta \mathbf{u})+\sum_{a=1}^{n} \widetilde{\omega}_{a}^{\mathrm{KK}},
$$

где $\widetilde{\omega}_{a}^{\mathrm{KK}}-$ форма на $\mathscr{O}_{a} / / \widetilde{\mathscr{G}}_{0}$. 


\section{6. Изомонодромные уравнения на эллиптических кривых}

В этом разделе мы обобщаем результаты работ [45], [46] в двух направлениях. Во-первых, на случай многих отмеченных точек. Отвечающие этому интегрируемые системы являются обобщениями эллиптических моделей Годена, описанных Н. Некрасовым в [32], где был рассмотрен простейший случай $\mathrm{SL}(N, \mathbb{C})$-расслоение с тривиальным характеристическим классом. Во-вторых, вместо интегрируемых систем мы описываем изомонодромные задачи. Соответствующие модели являются неавтономными гамильтоновыми системами. При этом будем использовать подход работ [28]-[30] к изомонодромным уравнениям, в котором эти уравнения трактуются как неавтономные обобщения систем Хитчина.

6.1. Деформации эллиптических кривых. Рассмотрим тор, т. е. $\widetilde{T}^{2}=$ $\{(x, y) \in \mathbb{R} \mid x, y \in \mathbb{R} / \mathbb{Z}\}$. Комплексная структура на $\widetilde{T}^{2}$ задается комплексными координатами $z=x+\tau_{0} y, \operatorname{Im} \tau_{0}>0$. Таким образом реализуем эллиптическую кривую $\Sigma_{\tau_{0}} \sim \mathbb{C} /\left(\mathbb{Z}+\tau_{0} \mathbb{Z}\right)$. Из (4.11) следует, что $\widetilde{T}^{2}$ должен иметь как минимум одну подвижную точку. Так как $\mathbb{C}$ действует на $\Sigma_{\tau_{0}}$ сдвигами $x \rightarrow x+c$, то в качестве отмеченной точки можно выбрать $x_{0}=0$, и остается единственный модуль деформации $\Sigma_{\tau_{0}} \rightarrow \Sigma_{\tau} \sim \mathbb{C} /(\mathbb{Z}+\tau \mathbb{Z})$. Мы считаем, что разность $\tau-\tau_{0}$ мала и отмеченные точки не совпадают. Большая клетка $\mathfrak{T}_{1, n}^{0}$ в пространстве времен есть пространство Тейхмюллера $\mathfrak{T}_{1, n}$, задаваемое как

$$
\mathfrak{T}_{1, n}^{0}=\left(\mathscr{H}^{+}=\{\operatorname{Im} \tau>0\} \times\left\{\left(x_{0}=0, \ldots, x_{n}\right), x_{k} \neq x_{j}(\bmod \langle\tau, 1\rangle)\right\}\right) .
$$

Следуя общему рецепту, введем новую координату

$$
w=z-\frac{\tau-\tau_{0}}{\rho_{0}}(\bar{z}-z), \quad \text { где } \rho_{0}=\tau_{0}-\bar{\tau}_{0} .
$$

При сдвигах $z \rightarrow z+1$ и $z \rightarrow z+\tau_{0}$ координата $w$ переходит в $w+1$ и $w+\tau$. Это означает, что $w$ - корректно определенная голоморфная координата на $\Sigma_{\tau}$. Однако такая деформация двигает точки $x_{a}$. Вместо нее рассмотрим

$$
w=z-\frac{\tau-\tau_{0}}{\rho_{0}}(\bar{z}-z)\left(1-\sum_{a=1}^{n} \chi_{a}(z, \bar{z})\right),
$$

где $\chi(z, \bar{z})$ - характеристическая функция $(4.7)$ и $\chi_{a}(z, \bar{z})=\chi\left(z-x_{a}^{0}, \bar{z}-\bar{x}_{a}^{0}\right)$. Как и в общем случае, мы используем координаты

$$
\left\{\begin{array}{l}
w=z-\frac{\tau-\tau_{0}}{\rho_{0}}(\bar{z}-z)\left(1-\sum_{a=1}^{n} \chi_{a}(z, \bar{z})\right), \\
\widetilde{w}=\bar{z}
\end{array}\right.
$$

Заметим, что при этом

$$
z+\tau_{0} \rightarrow\left(w+\tau, \widetilde{w}+\bar{\tau}_{0}\right)
$$


Принимая во внимание (4.9), определим

$$
\varepsilon(z, \bar{z})=\frac{t_{\tau}}{\rho_{0}}(\bar{z}-z)\left(1-\sum_{a=1}^{n} \chi_{a}(z, \bar{z})\right)+\sum_{a=1}^{n} t_{a} \chi_{a}(z, \bar{z}) .
$$

Деформированный оператор имеет вид $\partial_{\widetilde{w}}=\partial_{\bar{z}}+\mu \partial_{z}$, где $\mu$ (см. (4.9)) представляется следующей суммой:

$$
\mu=t_{\tau} \mu_{\tau}^{(0)}+\sum_{a=1}^{n} t_{a} \mu_{a}^{(0)}
$$

Из (4.2) и (6.2) находим $\mu_{\tau}^{(0)}$ :

$$
\mu_{\tau}^{(0)}=\frac{1}{\rho_{0}} \bar{\partial}(\bar{z}-z)\left(1-\sum_{a=1}^{n} \chi_{a}(z, \bar{z})\right), \quad t_{\tau}=\tau-\tau_{0},
$$

а из $(6.52) \mu_{a}^{(0)}$ :

$$
\mu_{a}^{(0)}=\bar{\partial} \chi_{a}(z, \bar{z}), \quad t_{a}=x_{a}-x_{a}^{0} .
$$

Базис, двойственный к дифференциалам Бельтрами $\mu_{\tau}^{(0)},\left\{\mu_{a}^{(0)}, a=1, \ldots, n\right\}$, относительно интегрирования по $\Sigma_{\tau}$, состоит из первых функций Эйзенштейна $E_{1}\left(z-x_{a}\right)(\mathrm{C} .2)$ и $1 . \mathrm{B}$ случае единственной отмеченной точки имеется только одно время $t_{\tau}$ (см. (4.11)).

Пространство модулей $\mathfrak{M}_{1}$ эллиптических кривых есть результат факторизации верхней полуплоскости $\mathscr{H}^{+}$по действию $\mathrm{SL}(2, \mathbb{Z})$ преобразованиями Мёбиуса:

$$
\mathfrak{M}_{1}=\mathscr{H}^{+} / \mathrm{SL}(2, \mathbb{Z}) \quad\left(\tau \rightarrow \frac{a \tau+b}{c \tau+d}\right) .
$$

Большая клетка $\mathfrak{M}_{1, n}^{0}$ на пространстве модулей определяется как фактор

$$
\mathfrak{M}_{1, n}^{0}=\mathfrak{T}_{1, n}^{0} / \mathrm{SL}(2, \mathbb{Z}) \quad\left(x_{a} \rightarrow x_{a}(c \tau+d)^{-1}\right)
$$

с $\mathfrak{T}_{1, n}^{0}$ из (6.1).

6.2. Симплектическая редукция. В общем случае $(0,1)$-часть связности в расслоении над эллиптическими кривыми может быть откалибрована на ноль (см. (4.21)):

$$
\bar{L}:=f^{-1} \partial_{\widetilde{w}} f+f^{-1} \bar{A} f=0 .
$$

Тогда $f$ действует на $A$ аналогично: $f^{-1} \partial_{w} f+f^{-1} A f=L$. Следовательно, уравнение момента принимает вид

$$
\partial_{\widetilde{w}} L_{\zeta}=\sum_{a=1}^{n} \mathbf{S}^{a} \delta\left(w-x_{a}\right)
$$

Это означает, что $L$ мероморфна и имеет простые полюсы в отмеченных точках с вычетами

$$
\operatorname{Res}_{w=x_{a}} L=\mathbf{S}^{a} \in \mathscr{O}_{a} \subset \mathfrak{g}^{*}, \quad \overrightarrow{\mathbf{S}}=\left(\mathbf{S}^{1}, \ldots, \mathbf{S}^{n}\right) .
$$


Для определения пространства $\widetilde{\mathrm{FBun}}_{\zeta}^{0}\left(\Sigma_{\tau}, \mathbf{x}, G\right)$ (5.30) следует принять во внимание условия квазипериодичности

$$
L_{\zeta}(w+1)=\operatorname{Ad}_{\mathscr{Q}} L(w), \quad L_{\zeta}(w+\tau)=\operatorname{Ad}_{\Lambda(\mathbf{u})} L(w) \quad\left(\left[\mathscr{Q}, \Lambda^{-1}(\mathbf{u})\right]=\zeta\right) .
$$

Тогда

$$
\begin{aligned}
& \widetilde{\operatorname{FBun}}_{\zeta}^{0}\left(\Sigma_{\tau}, \mathbf{x}, G\right)=\{\text { уравнения }(6.8) \text { с условиями }(6.10)\}, \\
& \operatorname{FBun}_{\zeta}^{0}\left(\Sigma_{\tau}, \mathbf{x}, G\right)=\{\text { уравнения }(6.8) \text { с условиями }(6.10)\} / \mathscr{G}_{\widetilde{\mathscr{H}_{0}}} .
\end{aligned}
$$

Линейная задача (4.27) на FBun ${ }_{\zeta}^{0}$ записывается в виде

$$
\begin{aligned}
& (\kappa \partial+L) \psi=0, \\
& \partial_{\widetilde{w}} \psi=0, \\
& \left(\kappa \partial_{s}+M_{s}\right) \psi=0 \quad(s=1, \ldots, n+1),
\end{aligned}
$$

где

$$
\partial_{\widetilde{w}}=\partial_{\bar{z}}+\left(t_{\tau} \mu_{\tau}^{(0)}+\sum_{a=1}^{n} t_{a} \mu_{a}^{(0)}\right) \partial_{z} .
$$

Наконец, уравнения Лакса имеют вид

$$
\kappa \partial_{s} L-\kappa \partial M_{s}+\left[M_{s}, L\right]=0 .
$$

6.3. Матрица Лакса. Для вычисления оператора $L$ будем использовать GS-базис (см. приложение B). Пусть $\left(\mathfrak{t}_{\beta}^{k}, \mathfrak{h}_{\alpha}^{k}\right\}$ - базис (B.5), (B.12) в $k$-компоненте $(5.11)$, а $\mathfrak{h}_{\alpha}^{0}$ - генераторы Картана в инвариантной подалгебре $\mathfrak{h}_{0}^{\prime}$. Рассмотрим вычеты (6.9) оператора $L$ :

$$
\mathbf{S}^{a}=\left(S^{a}\right)_{-\beta}^{\mathfrak{l},-k} \mathfrak{t}_{\beta}^{k}+\left(S^{a}\right)_{\alpha}^{\mathfrak{h},-k} \mathfrak{h}_{\alpha}^{k}+\left(S^{a}\right)_{\alpha}^{\mathfrak{h}, 0} \mathfrak{h}_{\alpha}^{0}
$$

Представим оператор $L$ как сумму картановской и корневой частей:

$$
L(w)=+L_{\mathfrak{h}}(w)+L_{\mathfrak{h}}^{0}(w)+L_{\mathrm{R}}(w)
$$

Тогда $L$, удовлетворяющий (6.8), (6.10), имеет вид

$$
\begin{aligned}
L_{\mathrm{R}}(w) & =\frac{1}{2} \sum_{a=1}^{n} \sum_{k=0}^{l-1} \sum_{\beta \in R}|\beta|^{2} \varphi_{\beta}^{k}\left(\mathbf{u}, w-x_{a}\right)\left(S^{a}\right)_{-\beta}^{\mathfrak{l},-k} \mathfrak{t}_{\beta}^{k}, \\
L_{\mathfrak{h}}(w) & =\sum_{a=1}^{n} \sum_{k=1}^{l-1} \sum_{\alpha \in \Pi} \phi\left(\frac{k}{l}, w-x_{a}\right)\left(S^{a}\right)_{\alpha}^{\mathfrak{h},-k} \mathfrak{h}_{\alpha}^{k}, \\
L_{\mathfrak{h}}^{0}(w) & =\sum_{\alpha \in \widetilde{\Pi}}\left(v_{\alpha}^{\mathfrak{h}}+\sum_{a=1}^{n} E_{1}\left(w-x_{a}\right)\left(S^{a}\right)_{\alpha}^{\mathfrak{h}, 0}\right) \mathfrak{h}_{\alpha}^{0} .
\end{aligned}
$$

Функции $\varphi_{\alpha}^{k}(\mathbf{u}, z)$ определены в (С.23), а $E_{1}(z)$ - функция Эйзенштейна (С.2). Необходимые свойства $L$ следуют из (С.19)-(С.21). 
Пуассонова структура. Опишем пуассонову структуру на пространствах $\widetilde{\operatorname{FBun}}_{\zeta}^{0}\left(\Sigma_{\tau}, \mathbf{x}, G\right)$ и $\operatorname{FBun}_{\zeta}^{0}\left(\Sigma_{\tau}, \mathbf{x}, G\right)(5.32)$. Указанное многообразие $\mathbf{P}$ является пуассоновым с каноническими скобками на $\mathbf{v}, \mathbf{u}$ и скобками Пуассона-Ли на $\mathbf{S}$ :

$$
\mathbf{P}=T^{*} C \times \bigcup_{a} \mathscr{O}_{a}=\left\{\mathbf{v}, \mathbf{u}, \mathbf{S}^{a} \in \mathscr{O}_{a}\right\}
$$

Его размерность равна $\sum_{a} \operatorname{dim}\left(\mathscr{O}_{a}\right)+2 \operatorname{dim}\left(\widetilde{\mathfrak{h}}_{0}\right)$. Опишем пуассонову структуру на $\operatorname{FBun}_{\zeta}^{0}$ как результат пуассоновой редукции относительно действия $\widetilde{\mathscr{G}}_{0}(5.19)$.

Рассмотрим пуассонову алгебру $\mathscr{A}=C^{\infty}(\mathbf{P})$ гладких функций на $\mathbf{P}$. Пусть $\varepsilon \in \widetilde{\mathfrak{h}}_{0}$, a $\gamma$ - малый контур вокруг $z=0$. Рассмотрим функцию

$$
\mu_{\varepsilon}=\oint_{\gamma}(\varepsilon, L(\mathbf{v}, \mathbf{u}, \mathbf{S}))=\left(\varepsilon, \mathbf{S}_{0}^{\mathfrak{h}}\right), \quad \mathbf{S}_{0}^{\mathfrak{h}}=\sum_{j=1}^{p} S_{j}^{\mathfrak{h}} e_{j} .
$$

Она генерирует векторное поле на $\mathbf{P}$ :

$$
V_{\varepsilon}: L(\mathbf{v}, \mathbf{u}, \mathbf{S}) \rightarrow\left\{\mu_{\varepsilon}, L(\mathbf{v}, \mathbf{u}, \mathbf{S})\right\}=[\varepsilon, L(\mathbf{v}, \mathbf{u}, \mathbf{S})] .
$$

Пусть $\mathscr{A}^{\text {inv }}$ есть инвариантная подалгебра $\mathscr{A}$ по действию $V_{\varepsilon}$. Тогда $I=$ $\left\{\mu_{\varepsilon} F(\mathbf{v}, \mathbf{u}, \mathbf{S}) \mid F(\mathbf{v}, \mathbf{u}, \mathbf{S}) \in \mathscr{A}\right\}-$ пуассонов идеал в $\mathscr{A}^{\text {inv }}$. Редуцированная пуассонова алгебра есть факторалгебра

$$
\mathscr{A}^{\mathrm{red}}=\mathscr{A}^{\mathrm{inv}} / I=\mathscr{A} \| \widetilde{\mathscr{G}}_{0} \quad\left(\widetilde{\mathscr{H}}_{0}=\exp \widetilde{\mathfrak{h}}_{0}\right) .
$$

Редуцированное пуассоново многообразие $\mathbf{P}^{\text {red }}$ определяется условием момента

$$
\sum_{a=1}^{n}\left(S^{a}\right)_{\alpha}^{\mathfrak{h}, 0}=0
$$

и $\operatorname{dim} \widetilde{\mathfrak{h}}$ условиями фиксации калибровки на спиновые переменные (эти условия мы не конкретизируем):

$$
\begin{aligned}
\mathbf{P}^{\text {red }} & =\mathbf{P} / / \widetilde{\mathscr{H}_{0}}=\mathbf{P}\left(\widetilde{S}_{s}^{\mathfrak{h}}=0\right) / \widetilde{\mathscr{H}_{0}} \\
\operatorname{dim}\left(\mathbf{P}^{\text {red }}\right) & =\operatorname{dim}(\mathbf{P})-2 \operatorname{dim}\left(\widetilde{\mathscr{H}_{0}}\right)=\operatorname{dim}(\mathscr{O}) .
\end{aligned}
$$

Вследствие условия момента из (6.15) получаем оператор Лакса, имеющий правильные (квази)периодические свойства. Он зависит от переменных $\left\{\mathbf{v}, \mathbf{u}, \mathbf{S}^{a}\right\}$ из $\mathbf{P}^{\text {red }}$. Здесь $\mathbf{S}^{a}$ не являются, вообе говоря, независимыми из-за наличия условий фиксации калибровки. Таким образом, после редукции приходим к пуассонову многообразию $\mathbf{P}^{\text {red }}$ с размерностью коприсоединенной орбиты $\mathscr{O}$. Скобки Пуассона на $\mathbf{P}^{\text {red }}$ вычисляются как скобки Дирака [84], [85].

На $\mathbf{P}^{\text {red }}$ уравнения движения записываются в форме Лакса (6.13). В пределе $\kappa \rightarrow 0$ возникающие изоспектральные потоки становятся вполне интегрируемыми, так как количество интегралов движения в инволюции равно $(1 / 2) \operatorname{dim}\left(\mathbf{P}^{\text {red }}\right)[45]$. 
6.4. Классическая $r$-матрица. Опишем пуассонову структуру на нередуцированном пространстве $\mathbf{P}$ в терминах классической динамической $r$-матрицы. Скобки Пуассона на $\mathbf{P}$ (см. (6.16)) представляются в виде прямой суммы скобок Пуассона-Ли в отмеченных точках и канонической скобки на $\mathbf{v}$ и $\mathbf{u}$ :

$$
\begin{aligned}
& \left\{\left(S^{a}\right)_{\alpha}^{\mathfrak{l}, k},\left(S^{b}\right)_{\beta}^{\mathfrak{l}, m}\right\}=\delta^{a b} \begin{cases}\frac{1}{\sqrt{l}} \sum_{s=0}^{l-1} \omega^{m s} C_{\alpha, \lambda^{s} \beta}\left(S^{a}\right)_{\alpha+\lambda^{s} \beta}^{\mathfrak{l}, k+m}, & \alpha \neq-\lambda^{s} \beta, \\
\frac{p_{\alpha}}{\sqrt{l}} \omega^{s m}\left(S^{a}\right)_{\alpha}^{\mathfrak{h}, k+m}, & \alpha=-\lambda^{s} \beta,\end{cases} \\
& \left\{\left(\bar{S}^{a}\right)_{\alpha}^{\mathfrak{h}, k},\left(S^{b}\right)_{\beta}^{\mathfrak{l}, m}\right\}=\delta^{a b} \frac{1}{\sqrt{l}} \sum_{s=0}^{l-1} \omega^{-k s}\left(\widehat{\alpha}, \lambda^{s} \beta\right)\left(S^{a}\right)_{\beta}^{\mathfrak{l}, k+m} \\
& \left\{\bar{v}_{\alpha}^{\mathfrak{h}}, u_{\beta}\right\}=\frac{1}{\sqrt{l}} \sum_{s=0}^{l-1}\left(\widehat{\alpha}, \lambda^{s} \beta\right) \\
& \left\{v_{\alpha},\left(S^{a}\right)_{\alpha}^{\mathfrak{l}, k}\right\}=\left\{v_{\alpha},\left(S^{a}\right)_{\alpha}^{\mathfrak{h}, k}\right\}=\left\{v_{\alpha},\left(S^{a}\right)_{\alpha}^{\mathfrak{h}, k}\right\}=0, \\
& \left\{u_{\alpha},\left(S^{a}\right)_{\alpha}^{\mathfrak{l}, k}\right\}=\left\{u_{\alpha},\left(S^{a}\right)_{\alpha}^{\mathfrak{h}, k}\right\}=\left\{u_{\alpha},\left(S^{a}\right)_{\alpha}^{\mathfrak{h}, k}\right\}=0 .
\end{aligned}
$$

Скобки Пуассона-Ли в каждой точке двойственны коммутационным соотношениям (В.39), (В.40) в алгебре Ли. Скобки (6.19) воспроизводятся динамической $r$-матричной структурой с $r$-матрицей

$$
r(\mathbf{u}, z, w)=r(\mathbf{u}, z-w)=r_{\mathfrak{h}}(\mathbf{u}, z-w)+r_{\mathrm{R}}(\mathbf{u}, z-w),
$$

где

$$
\begin{aligned}
& r_{\mathrm{R}}(\mathbf{u}, z)=\frac{1}{2} \sum_{k=0}^{l-1} \sum_{\alpha \in R}|\alpha|^{2} \varphi_{\alpha}^{k}(\mathbf{u}, z) \mathfrak{t}_{\alpha}^{k} \otimes \mathfrak{t}_{-\alpha}^{-k}, \\
& r_{\mathfrak{h}}(\mathbf{u}, z)=\sum_{k=0}^{l-1} \sum_{\alpha \in \Pi} \varphi_{0}^{k}(\mathbf{u}, z) \mathfrak{h}_{\alpha}^{k} \otimes \mathfrak{h}_{\alpha}^{-k} .
\end{aligned}
$$

Более точно, следующие два утверждения были доказаны в [45].

ПРЕДЛОЖЕНИЕ 6.1. г-матрица (6.20)-(6.21) и описанный выше оператор Лакса (6.14)-(6.15) определяют скобки Пуассона (6.19) через RLL-уравнение:

$$
\begin{aligned}
\{L(w) \otimes 1,1 \otimes L(w)\}=[ & L(w) \otimes 1+1 \otimes L(w), r(z, w)] \\
& \quad-\frac{\sqrt{l}}{2} \sum_{k=0}^{l-1} \sum_{\alpha \in R}|\alpha|^{2} \partial_{\mathbf{u}} \varphi_{\alpha}^{k}(\mathbf{u}, z-w) \bar{S}_{\alpha}^{\mathfrak{h}, 0} t_{\alpha}^{k} \otimes t_{-\alpha}^{-k}
\end{aligned}
$$

Тождество Якоби на скобки (6.22) вытекает из следующего предложения. 
ПРЕДЛОЖЕНИЕ 6.2. r-матрииа (6.20)-(6.21) удовлетворяет классическому динамическому уравнению Янга-Бакстера:

$$
\begin{aligned}
& {\left[r_{12}(z, w), r_{13}(z, x)\right]+} {\left[r_{12}(z, w), r_{23}(w, x)\right]+\left[r_{13}(z, x), r_{23}(w, x)\right] } \\
&-\sqrt{l} \sum_{k=0}^{l-1} \sum_{\alpha \in R}\left(\frac{|\alpha|^{2}}{2} \mathfrak{t}_{\alpha}^{k} \otimes \mathfrak{t}_{-\alpha}^{-k} \otimes \overline{\mathfrak{h}}_{\alpha}^{0} \partial_{\mathbf{u}} \varphi_{\alpha}^{k}(\mathbf{u}, z-w)\right. \\
&-\frac{|\alpha|^{2}}{2} \mathfrak{t}_{\alpha}^{k} \otimes \overline{\mathfrak{h}}_{\alpha}^{0} \otimes \mathfrak{t}_{-\alpha}^{-k} \partial_{\mathbf{u}} \varphi_{\alpha}^{k}(\mathbf{u}, z-x) \\
&\left.+\frac{|\alpha|^{2}}{2} \overline{\mathfrak{h}}_{\alpha}^{0} \otimes \mathfrak{t}_{\alpha}^{k} \otimes \mathfrak{t}_{-\alpha}^{-k} \partial_{\mathbf{u}} \varphi_{\alpha}^{k}(\mathbf{u}, w-x)\right)=0 .
\end{aligned}
$$

Последний член в правой части (6.22) нарушает интегрируемость на $\mathbf{P}$. Тем не менее действие подгруппы Картана $\mathscr{H}_{0}^{\prime}$ из инвариантной подгруппы $G_{0}^{\prime} \subset G$ генерирует отображение момента:

$$
\sum_{a=1}^{n}\left(S^{a}\right)_{\alpha}^{\mathfrak{h}, 0}=0 \quad \forall \alpha \in \widetilde{\Pi} .
$$

Как уже объяснялось, после редукции по $\widetilde{\mathscr{H}}_{0}(6.18)$ получаем $\mathbf{P}^{\text {red }}$, на котором нарушающее интегрируемость слагаемое зануляется. Тогда

$$
\left\{L^{\mathrm{red}}(w) \otimes 1,1 \otimes L^{\mathrm{red}}(w)\right\}=\left[L^{\mathrm{red}}(w) \otimes 1+1 \otimes L^{\mathrm{red}}(w), \widetilde{r}(z, w)\right] .
$$

Здесь $r$-матрица заменена на $\widetilde{r}$, так как пуассонова структура на $\mathbf{P}^{\text {red }}$ отличается от исходной на $\mathbf{P}$. Разница возникает из дираковских членов в редукции $\mathbf{P}^{\text {red }}=\mathbf{P} / / \widetilde{\mathscr{H}}_{0}$. Нам не требуется здесь ее явное описание.

Классические динамические $r$-матрицы, отвечающие тривиальным расслоениям, были построены в [86]. Динамический параметр u принадлежит подалгебре Картана $\mathfrak{h} \subset \mathfrak{g}$. Задача классификации $r$-матриц в случаях $\mathbf{u} \in \widetilde{\mathfrak{h}} \subset \mathfrak{h}$ была поставлена в [86]. В тригонометрическом случае без спектрального параметра ее решение было предложено в [87] в терминах симметрий расширенных графов Дынкина. Эллиптическая версия была рассмотрена в [88], [89] и [45].

6.5. Симметрии фазового пространства. Явный вид оператора Лакса (6.15) позволяет описать действие группы Бернштейна-Шварцмана на динамических переменных. Как объяснялось выше, это действие генерируется калибровочной подгруппой $\mathscr{G}_{\widetilde{H}_{0}}(5.20)$. Вдобавок рассмотрим действие модулярной группы $\operatorname{SL}(2, \mathbb{Z})=\left\{\left(\begin{array}{ll}a & b \\ c & d\end{array}\right)\right\}$. Для вычисления модулярных преобразований спиновых переменных используем уравнение момента $\sum_{a}\left(\mathbf{S}^{a}\right)_{-\alpha}^{\mathfrak{h}, 0}=0$ и соотношения (С.42)-(С.44). Заметим, что оператор Лакса неинвариантен относительно действия модулярной группы. Действительно, из (С.42)-(С.44) имеем

$$
\begin{aligned}
& \varphi_{\alpha}^{k}\left(\frac{\mathbf{u}}{c \tau+d}, \frac{z}{c \tau+d} \mid \frac{a \tau+b}{c \tau+d}\right) \equiv \exp (2 \pi i z\langle\varrho, \alpha\rangle) \phi\left(\langle\mathbf{u}, \alpha\rangle+\tau\langle\varrho, \alpha\rangle+\frac{k}{l}, z \mid \tau\right) \\
& =\exp \left(2 \pi i z\left(\frac{c\langle\mathbf{u}, \alpha\rangle}{c \tau+d}+\langle\varrho, \alpha\rangle a+\frac{k}{l} c\right)\right) \phi\left(\langle\mathbf{u}, \alpha\rangle+\left(\langle\varrho, \alpha\rangle, \frac{k}{l}\right)\left(\begin{array}{ll}
a & b \\
c & d
\end{array}\right)\left(\begin{array}{l}
\tau \\
1
\end{array}\right), z \mid \tau\right) \\
& =\exp \left(2 \pi i z \frac{c\langle\mathbf{u}, \alpha\rangle}{c \tau+d}\right) \varphi_{\alpha^{\prime}}^{k^{\prime}}(\mathbf{u}, z \mid \tau),
\end{aligned}
$$


где последнее равенство следует рассматривать как определение $k^{\prime}$ и $\alpha^{\prime}$, т. е.

$$
\left\langle\mathbf{u}, \alpha^{\prime}\right\rangle+\tau\left\langle\varrho, \alpha^{\prime}\right\rangle+\frac{k^{\prime}}{l}=\langle\mathbf{u}, \alpha\rangle+\left(\langle\varrho, \alpha\rangle, \frac{k}{l}\right)\left(\begin{array}{ll}
a & b \\
c & d
\end{array}\right)\left(\begin{array}{l}
\tau \\
1
\end{array}\right) .
$$

Тем не менее операторы Лакса становятся инвариантными относительно действия модулярной группы, если оно сопровождается некоторым калибровочным преобразованием. Результаты, приведенные в табл. 1, даны с учетом такого преобразования. Во-первых, множитель $\exp \left(2 \pi i z \frac{c\langle\mathbf{u}, \alpha\rangle}{c \tau+d}\right)$ можно откалибровать. Это делается с помощью $h=h(\mathbf{u}, z)$ из подгруппы Картана. Во-вторых, подействуем $g$ таким, что $\operatorname{Ad}_{g} \mathfrak{t}_{\alpha}^{k}=\mathfrak{t}_{\alpha^{\prime}}^{k^{\prime}}$. В итоге имеем:

$$
\kappa \partial_{z}+L\left(\mathbf{S}, \mathbf{v}, \frac{\mathbf{u}}{c \tau+d}, \frac{z}{c \tau+d} \mid \frac{a \tau+b}{c \tau+d}\right)=\operatorname{Ad}_{g h}\left(\kappa \partial_{z}+L\left(\mathbf{S}^{\prime}, \mathbf{v}^{\prime}, \mathbf{u}^{\prime}, z \mid \tau\right)\right),
$$

где штрихованные переменные приводятся в последнем (правом) столбце табл. 1.

ТАБлицА 1. Симметрии переменных фазового пространства

\begin{tabular}{|c|c|c|c|}
\hline & $\widetilde{W}_{0}=\{s\}$ & $\widetilde{R}^{\vee} \oplus \tau R^{\vee}$ & $\mathrm{SL}(2, \mathbb{Z})$ \\
\hline \hline $\mathbf{v}$ & $s \mathbf{v}$ & $\mathbf{v}+\kappa \gamma$ & $\mathbf{v}(c \tau+d)+2 \pi i \kappa \frac{c \mathbf{u}}{c \tau+d}$ \\
\hline $\mathbf{u}$ & $s \mathbf{u}$ & $\mathbf{u}+\gamma_{1}+\gamma_{2} \tau$ & $-2 \pi i c \sum_{a} x_{a}\left(\mathbf{S}^{a}\right)^{\mathfrak{h}, 0}$ \\
\hline$\left(\mathbf{S}^{a}\right)_{-\alpha}^{\mathfrak{h},-k}$ & $\left(\mathbf{S}^{a}\right)_{-s \alpha}^{\mathfrak{h},-k}$ & $\left(\mathbf{S}^{a}\right)_{-\alpha}^{\mathfrak{h},-k}$ & $\mathbf{u}(c \tau+d)^{-1}$ \\
\hline$\left(\mathbf{S}^{a}\right)_{-\alpha}^{\mathfrak{h}, 0}$ & $\left(\mathbf{S}^{a}\right)_{-s \alpha}^{\mathfrak{h}, 0}$ & $\left(\mathbf{S}^{a}\right)_{-\alpha}^{\mathfrak{h}, 0}$ & $(c \tau+d)\left(\mathbf{S}^{a}\right)_{-\alpha^{\prime}}^{\mathfrak{h},-k^{\prime}}$ \\
\hline$\left(\mathbf{S}^{a}\right)_{-\alpha}^{\mathfrak{l}, k}$ & $\left(\mathbf{S}^{a}\right)_{-s \alpha}^{\mathfrak{l}, k}$ & $\chi_{\langle\gamma, \alpha\rangle}\left(x_{a}\right)\left(\mathbf{S}^{a}\right)_{-\alpha}^{\mathfrak{l}, k}$ & $(c \tau+d) \exp \left(-2 \pi i \frac{c x_{a}\langle\mathbf{u}, \alpha\rangle}{c \tau+d}\right)\left(\mathbf{S}_{-\alpha}^{a}\right)_{-\alpha^{\prime}}^{\mathfrak{l}, k^{\prime}}$ \\
\hline$\tau$ & $\tau$ & $\tau$ & $\frac{a \tau+b}{c \tau+d}$ \\
\hline$x_{a}$ & $x_{a}$ & $x_{a}$ & $x_{a}(c \tau+d)^{-1}$ \\
\hline
\end{tabular}

Из последнего столбца следует, что можно рассматривать независимые переменные, принимающие значения на пространстве

$$
\mathfrak{M}_{1, n}=\mathfrak{T}_{1, n} / \mathrm{SL}(2, \mathbb{Z})
$$

модулей эллиптических кривых с отмеченными точками. Расслоение Пуанкаре-Картана (4.16) становится нетривиальным над $\mathfrak{M}_{1, n}$ :

$$
\begin{aligned}
& \mathscr{P}(G) \\
& \underset{\widetilde{\operatorname{FBun}}_{\zeta}^{0}}{0}\left(\Sigma_{\tau}, \mathbf{x}, G\right)(6.11) \\
& \mathfrak{M}_{1, n}
\end{aligned}
$$


6.6. Гамильтонианы. Рассмотрим расширенное фазовое пространство $\mathscr{P}$ (4.16) для расслоений над эллиптическими кривыми. Заменим пространство Тейхмюллера $\mathscr{H}^{+}=\{\tau \mid \operatorname{Im} \tau>0\}$ на факторпространство $-\mathscr{H}^{+} / \operatorname{SL}(2, \mathbb{Z})$. Пусть

$$
\mathfrak{M}_{n}=\left\{\left(\tau, \mathbf{x}=\left(x_{1}, \ldots, x_{n}\right)\right) \mid \tau \in \mathscr{H}^{+} / \mathrm{SL}(2, \mathbb{Z}), x_{a} \neq x_{b}\right\}
$$

Тогда пространство $\mathscr{P}$ имеет структуру

$$
\begin{aligned}
& \mathscr{P}(G) \\
& \downarrow_{\mathscr{R}_{\zeta}\left(\Sigma_{\tau}, \mathbf{x}, G\right)} \\
& \mathfrak{M}_{n}
\end{aligned}
$$

Локальная координата в слое $\mathscr{R}_{\zeta}\left(\Sigma_{\tau}, \mathbf{x}, G\right)$ есть $(\mathbf{v}, \mathbf{u}, \overrightarrow{\mathbf{S}})$ с симплектической формой $\omega^{\mathscr{R}}(5.31)$.

Вычислим гамильтонианы (4.24) через дифференциал Бельтрами (6.4), (6.5). В координатах $(w, \widetilde{w})$ плотность меры на $\Sigma_{\tau}$ имеет вид

$$
d \sigma=\frac{d z d \bar{z}}{\rho_{0}}=\frac{d w d \widetilde{w}}{\rho}, \quad \rho_{0}=\tau_{0}-\bar{\tau}_{0}, \quad \rho=\tau-\bar{\tau}_{0} .
$$

Из вида $L$ следует, что при условии (6.24) $\operatorname{tr}\left(L^{2}(w)\right)$ - периодическая функция на $\Sigma_{\tau}$ с полюсами второго порядка в отмеченных точках (6.9). Следовательно, гамильтонианы могут быть вычислены из разложения

$$
\frac{1}{2} \operatorname{tr}\left(L^{2}(w)\right)=H_{\tau}+\sum_{a=1}^{n}\left(H_{a} E_{1}\left(w-x_{a}\right)+C_{2}^{a} E_{2}\left(w-x_{a}\right)\right)
$$

или через интегралы

$$
H_{\tau}=\frac{1}{2} \int_{\Sigma_{\tau}} \operatorname{tr}\left(L^{2}\right) \mu_{\tau}^{0}, \quad H_{a}=\frac{1}{2} \int_{\Sigma_{\tau}} \operatorname{tr}\left(L^{2}\right) \mu_{a}^{0} .
$$

Квадратичные функции Казимира (отвечающие орбитам в точках $x_{a}$ ) суть

$$
C_{2}^{a}=\frac{1}{2} \sum_{k=0}^{l-1} \sum_{\alpha \in R}|\alpha|^{2}\left(S^{a}\right)_{\alpha}^{\mathfrak{l}, k}\left(S^{a}\right)_{-\alpha}^{\mathfrak{l},-k}+\sum_{k=0}^{l-1} \sum_{\alpha \in \Pi}\left(S^{a}\right)_{\alpha}^{\mathfrak{h}, k}\left(S^{a}\right)_{\alpha}^{\mathfrak{h},-k}, \quad a=1, \ldots, n .
$$

Гамильтонианы системы Годена (или системы Шлезингера) имеют вид

$$
\begin{aligned}
H_{a}=l & \sum_{\alpha \in \Pi} v_{\alpha}^{\mathfrak{h}}\left(S^{a}\right)_{\alpha}^{\mathfrak{h}, 0}+\sum_{c \neq a}^{n} \sum_{k=0}^{l-1}\left(\frac{1}{2} \sum_{\alpha \in R}|\alpha|^{2} \varphi_{\alpha}^{k}\left(\mathbf{u}, z_{a}-z_{c}\right)\left(S^{a}\right)_{\alpha}^{\mathfrak{l}, k}\left(S^{c}\right)_{-\alpha}^{\mathfrak{l},-k}\right. \\
& \left.+\sum_{\alpha \in \Pi} \varphi_{0}^{k}\left(\mathbf{u}, z_{a}-z_{c}\right)\left(S^{a}\right)_{\alpha}^{\mathfrak{h}, k}\left(S^{c}\right)_{\alpha}^{\mathfrak{h},-k}\right) .
\end{aligned}
$$


Наконец, нулевой гамильтониан, отвечающий за взаимодействие частиц, имеет вид

$$
\begin{aligned}
H_{\tau}= & \frac{l}{2} \sum_{\alpha \in \Pi} \sum_{s=0}^{l-1} v_{\alpha}^{\mathfrak{h}} v_{\lambda^{s} \widehat{\alpha}}^{\mathfrak{h}}+\sum_{b \neq d}^{n} \sum_{k=0}^{l-1}\left(\frac{1}{2} \sum_{\alpha \in R}|\alpha|^{2} f_{\alpha}^{k}\left(\mathbf{u}, z_{b}-z_{d}\right)\left(S^{b}\right)_{\alpha}^{\mathfrak{l}, k}\left(S^{d}\right)_{-\alpha}^{\mathfrak{l},-k}\right. \\
& \left.+\sum_{\alpha \in \Pi} f_{0}^{k}\left(\mathbf{u}, z_{b}-z_{d}\right)\left(S^{b}\right)_{\alpha}^{\mathfrak{h}, k}\left(S^{d}\right)_{\alpha}^{\mathfrak{h},-k}\right)
\end{aligned}
$$

Функции $f_{\alpha}^{k}(\mathbf{u}, z)$ определены в (С.26)-(С.28).

6.7. $M$-операторы. $M_{s}$-операторы могут быть вычислены из уравнений (6.13), так как $L$-оператор уже известен. Мы построим их иначе. Следуя рецепту из [90], получим $M$-операторы из $r$-матричной структуры $(6.22)$ с $r$-матрицей (6.20)-(6.21).

Рассмотрим сначала автономный случай (4.29). Определим пары Лакса для интегрируемых потоков гамильтонианов (6.31), (6.32). Вычислим скобки:

$$
\frac{1}{2} \operatorname{tr}_{2}\left\{1 \otimes L^{2}(z), L(z) \otimes 1\right\}=\left[L(z), M_{w}(z)\right]+\Delta(z, w)
$$

где $\operatorname{tr}_{2}$ - след по второй компоненте в тензорном произведении

$$
M_{w}(z)=-\operatorname{tr}_{2}\left(r(z, w) L_{2}(w)\right)
$$

a $\Delta(z, w)$ - слагаемое от последнего члена в (6.22):

$$
\Delta(z, w)=\operatorname{tr}_{2}\left(\frac{\sqrt{l}}{2} \sum_{k=0}^{l-1} \sum_{\alpha \in R}|\alpha|^{2} \partial_{\mathbf{u}} \varphi_{\alpha}^{k}(\mathbf{u}, z-w) \bar{S}_{\alpha}^{\mathfrak{h}, 0} t_{\alpha}^{k} \otimes t_{-\alpha}^{-k} L_{2}(z)\right) .
$$

Этот член зануляется на поверхности связей первого рода (6.24). Тогда на (6.24) имеем

$$
\left.\frac{1}{2} \operatorname{tr}_{2}\left\{1 \otimes L^{2}(z), L(z) \otimes 1\right\}\right|_{(6.24)}=\left[L(z), M_{w}(z)\right] .
$$

Учитывая (6.29), получаем, что $M$-операторы гамильтонианов (6.31), (6.32) вычисляются разложением $M_{w}(w)$ (6.34) аналогично (6.29). Таким образом, получим набор уравнений Лакса

$$
\partial_{t_{a}} L(z)=\left[L(z), M_{a}(z)\right], \quad a=1, \ldots, n,
$$

И

$$
\partial_{t_{\tau}} L(z)=\left[L(z), M_{\tau}(z)\right]
$$

на поверхности связей (6.24). Эти $M$-операторы имеют вид

$$
M_{a}(z)=\left(M^{a}\right)_{\mathrm{R}}(z)+\left(M^{a}\right)_{\mathfrak{h}}(z)+\left(M^{a}\right)_{\mathfrak{h}}^{0}(z)
$$


где

$$
\begin{aligned}
\left(M^{a}\right)_{\mathrm{R}}(z) & =-\frac{1}{2} \sum_{k=0}^{l-1} \sum_{\beta \in R}|\beta|^{2} \varphi_{\beta}^{k}\left(\mathbf{u}, z-x_{a}\right)\left(S^{a}\right)_{-\beta}^{\mathfrak{l},-k} \mathfrak{t}_{\beta}^{k}, \\
\left(M^{a}\right)_{\mathfrak{h}}(z) & =-\sum_{k=1}^{l-1} \sum_{\alpha \in \Pi} \phi\left(\frac{k}{l}, z-x_{a}\right)\left(S^{a}\right)_{\alpha}^{\mathfrak{h},-k} \mathfrak{h}_{\alpha}^{k}, \\
\left(M^{a}\right)_{\mathfrak{h}}^{0}(z) & =-\sum_{\alpha \in \Pi} E_{1}\left(z-x_{a}\right)\left(S^{a}\right)_{\alpha}^{\mathfrak{h}, 0} \mathfrak{h}_{\alpha}^{0}
\end{aligned}
$$

и

$$
M^{\tau}(z)=\left(M^{\tau}\right)_{\mathrm{R}}(z)+\left(M^{\tau}\right)_{\mathfrak{h}}(z)+\left(M^{\tau}\right)_{\mathfrak{h}}^{0}(z)
$$

где

$$
\begin{aligned}
\left(M^{\tau}\right)_{\mathrm{R}}(z) & =\frac{1}{2} \sum_{a=1}^{n} \sum_{k=0}^{l-1} \sum_{\beta \in R}|\beta|^{2} f_{\beta}^{k}\left(\mathbf{u}, z-x_{a}\right)\left(S^{a}\right)_{-\beta}^{\mathfrak{l},-k} \mathfrak{t}_{\beta}^{k}, \\
\left(M^{\tau}\right)_{\mathfrak{h}}(z) & =\sum_{a=1}^{n} \sum_{k=1}^{l-1} \sum_{\alpha \in \Pi} f\left(\frac{k}{l}, z-x_{a}\right)\left(S^{a}\right)_{\alpha}^{\mathfrak{h},-k} \mathfrak{h}_{\alpha}^{k}, \\
\left(M^{\tau}\right)_{\mathfrak{h}}^{0}(z) & =\frac{1}{2} \sum_{a=1}^{n} \sum_{\alpha \in \Pi}\left(E_{1}^{2}\left(z-x_{a}\right)-\wp\left(z-x_{a}\right)\right)\left(S^{a}\right)_{\alpha}^{\mathfrak{h}, 0} \mathfrak{h}_{\alpha}^{0} .
\end{aligned}
$$

Как отмечалось, связи (6.24) должны быть дополнены условиями фиксации калибровки (действия подгруппы Картана $\mathscr{H}_{0}$ инвариантной подгруппы). Полная редукция $\mathbf{P}^{\text {red }}=\mathbf{P} / / \widetilde{\mathscr{H}}_{0}$ (вообще говоря) меняет $M$-операторы и $r$-матрицы (см. пример бесспиновой системы Калоджеро в [91]). Мы не рассматриваем здесь полные редукции, так как в общем случае нет "хорошего" выбора фиксации калибровки.

6.8. Уравнения Пенлеве-Шлезингера. В начале раздела отмечалось, что изомонодромные уравнения в нашем подходе возникают как неавтономные обобщения систем Хитчина [30]. На уровне уравнений Лакса или нулевой кривизны это означает, что пары Лакса, удовлетворяющие (6.37) или (6.38), удовлетворяет и изомонодромным уравнениям

$$
\partial_{x_{a}} L(z)-\partial_{z} M_{a}(z)=\left[L(z), M_{a}(z)\right], \quad a=1, \ldots, n,
$$

или

$$
\partial_{\tau} L(z)-\partial_{z} M_{\tau}=\left[L(z), M_{\tau}(z)\right]
$$

соответственно. Это явление известно как соответствие Калоджеро-Пенлеве [44] (см. развитие этой идеи в [92] и квантовую версию в [93]-[96]).

Заметим, что временные переменные $t_{a}$ в (6.37) заменяются на координаты отмеченных точек $x_{a}$ в $(6.43)$, а матрицы $M_{a}(z)$ одинаковы для обоих наборов уравнений. Аналогично, временная переменная $t_{\tau}$ в $(6.38)$ заменяется на модуль $\tau$ в $(6.44)$, и точно также $M_{\tau}$ одинаков в обоих случаях. Технически соответствие Калоджеро-Пенлеве следует из

$$
d_{x_{a}} L(z)=\partial_{z} M_{a}(z),
$$


где $d_{x_{a}}-$ производная только по явной зависимости от $x_{a}$, и

$$
d_{\tau} L(z)=\partial_{z} M_{\tau}
$$

где $d_{\tau}$ - производная только по явной зависимости от $\tau$. Уравнения $(6.45)$ тривиально следуют из вида зависимости $L\left(z, x_{a}\right)=L\left(z-x_{a}\right), M_{a}\left(z, x_{a}\right)=$ $M_{a}\left(z-x_{a}\right)$, а (6.46) следует из уравнения теплопроводности (С.29).

Таким образом, получим уравнения Пенлеве-Шлезингера (6.43) и (6.44). Отметим также, что $M$-оператор можно вывести в терминах $\Xi(z)$ из предположения о существовании соответствия Калоджеро-Пенлеве [97].

\section{9. Уравнения Книжника-Замолодчикова-Бернара.}

6.9.1. Уравнения Книжника-Замолодчикова-Бернара на эллиптических кривых. Уравнения Книжника-Замолодчикова-Бернара (КЗБ) возникают как условие горизонтальности некоторых сечений (конформных блоков) для так называемой КЗБ-связности. Уравнения КЗБ (см. (6.52) ниже) можно рассматривать как квантование изомонодромных уравнений. КЗБ-связность на эллиптической кривой можно построить, следуя [98]. В случае произвольного характеристического класса она задается следующими дифференциальными операторами [48]:

$$
\begin{aligned}
& \nabla_{a}=\partial_{z_{a}}+\widehat{\partial}^{a}+\sum_{c \neq a} r^{a c} \\
& \nabla_{\tau}=2 \pi i \partial_{\tau}+\Delta+\frac{1}{2} \sum_{b, d} f^{b d}
\end{aligned}
$$

где

$$
\begin{aligned}
r^{a c} & =\frac{1}{2} \sum_{k=0}^{l-1} \sum_{\alpha \in R}|\alpha|^{2} \varphi_{\alpha}^{k}\left(\mathbf{u}, z_{a}-z_{c}\right) \mathfrak{t}_{\alpha}^{k, a} \mathfrak{t}_{-\alpha}^{-k, c}+\sum_{k=0}^{l-1} \sum_{\alpha \in \Pi} \varphi_{0}^{k}\left(\mathbf{u}, z_{a}-z_{c}\right) \mathfrak{H}_{\alpha}^{k, a} \mathfrak{h}_{\alpha}^{k, c}, \\
f^{a c} & =\frac{1}{2} \sum_{k=0}^{l-1} \sum_{\alpha \in R}|\alpha|^{2} f_{\alpha}^{k}\left(\mathbf{u}, z_{a}-z_{c}\right) \mathfrak{t}_{\alpha}^{k, a} \mathfrak{t}_{-\alpha}^{-k, c}+\sum_{k=0}^{l-1} \sum_{\alpha \in \Pi} f_{0}^{k}\left(\mathbf{u}, z_{a}-z_{c}\right) \mathfrak{H}_{\alpha}^{k, a} \mathfrak{h}_{\alpha}^{k, c},
\end{aligned}
$$

$\mathfrak{t}_{\alpha}^{k, a}=1 \otimes \cdots 1 \otimes \mathfrak{t}_{\alpha}^{k} \otimes 1 \cdots \otimes 1\left(\mathbf{c}_{\alpha}^{k}\right.$ на $a$-м месте) и аналогично для генераторов $\mathfrak{H}_{\alpha}^{k, a}$ и $\mathfrak{h}_{\alpha}^{k, a 6}$. Используем обозначения

$$
\widehat{\partial}^{a}=l \sum_{\alpha \in \Pi} \mathfrak{h}_{\alpha}^{0, a} \partial_{\widehat{\alpha}}, \quad \Delta=\frac{l}{2} \sum_{\alpha \in \Pi} \sum_{s=0}^{l-1} \partial_{u_{\alpha}} \partial_{u_{\lambda^{s}} \widehat{\alpha}} .
$$

Из определения следует, что $r^{a c}=-r^{c a}$ и $f^{a c}=f^{c a}$. Заметим, что

$$
f^{c c}=-\sum_{k=0}^{l-1} \sum_{\alpha \in R}|\alpha|^{2} \wp_{\alpha}^{k} \mathfrak{t}_{\alpha}^{k, c} \mathfrak{t}_{-\alpha}^{-k, c}-\sum_{k=0}^{l-1} \sum_{\alpha \in \Pi} \mathfrak{H}_{\alpha}^{k, c} \mathfrak{h}_{\alpha}^{-k, c}-2 l \eta_{1} C_{2}^{c},
$$

\footnotetext{
${ }^{6}$ Для краткости пишем $\mathfrak{t}_{\alpha}^{k, a}, \mathfrak{h}_{\alpha}^{k, a}$ вместо представлений этих генераторов в пространствах $V_{\mu_{a}}$.
} 
где $C_{2}^{c}$ - оператор Казимира, действующий на $c$-ю компоненту. Уравнения КЗБ имеют вид

$$
\left\{\begin{array}{l}
\nabla_{a} F=0, \quad a=1, \ldots, n, \\
\nabla_{\tau} F=0 .
\end{array}\right.
$$

Условия совместности делятся на два типа (6.52):

$$
\left\{\begin{array}{l}
{\left[\nabla_{a}, \nabla_{b}\right] F=0, \quad a, b=1, \ldots, n,} \\
{\left[\nabla_{a}, \nabla_{\tau}\right] F=0, \quad a=1, \ldots, n .}
\end{array}\right.
$$

Важно отметить, что решения $F$ уравнений $(6.52)$ удовлетворяют условию (cм. (6.24))

$$
\left(\sum_{c=1}^{n} \mathfrak{h}_{\alpha}^{0, c}\right) F=0 \quad \text { для любого } \alpha \in \widetilde{\Pi} .
$$

ПРеДЛОЖЕНИЕ 6.3. Верхние уравнения $\left[\nabla_{a}, \nabla_{b}\right]=0$ из (6.53) выполняются для г-матрицы (6.20) на решениях (6.52), удовлетворяющих (6.54). Это является следствием классического динамического уравнения Янга-Бакстера:

$$
\left[r^{a b}, r^{a c}\right]+\left[r^{a b}, r^{b c}\right]+\left[r^{a c}, r^{b c}\right]+\left[\widehat{\partial}^{a}, r^{b c}\right]+\left[\widehat{\partial}^{c}, r^{a b}\right]+\left[\widehat{\partial}^{b}, r^{c a}\right]=0
$$

ПРЕДЛОЖЕНИЕ 6.4. Нижние уравнения $\left[\nabla_{a}, \nabla_{\tau}\right]=0$ из (6.53) также выполняются для г-матрищы (С.23) на решениях (6.52), удовлетворяющих (6.54).

Доказательство приведенных утверждений изложено в [48].

6.9.2. Уравнения КЗБ и изомонодромная задача. Уравнения КЗБ (6.47), (6.48), написанные здесь в эллиптическом случае, могут быть сформулированы и для кривой произвольного рода [99]-[101]. Их связь с задачей на сфере исследовалась в [102], [103]. В общем случае, как и на торе, уравнения КЗБ имеют форму нестационарного уравнения Шрёдингера

$$
\left(\kappa \partial_{s}+\widehat{H}_{s}\right) \Psi=0
$$

с квантовыми квадратичными хитчиновскими гамильтонианами $\widehat{H}_{s}$. Для перехода к классическому пределу заменим волновую функцию (конформный блок) квазиклассическим выражением

$$
\Psi=\exp \left(\frac{i S}{\kappa}\right)
$$

где $S$ - классическое действие $(S=\log \tau(4.19))$. Классический предел $\kappa \rightarrow 0$ дает уравнения Гамильтона-Якоби $S$ [104]. Они эквивалентны уравнениям движения (4.26a). С другой стороны, полагая $\kappa=0$ в (6.56), получаем стационарные уравнения Шрёдингера $\widehat{H}_{s} \Psi=0$. На классическом уровне предел $\kappa \rightarrow 0$ 
воспроизводит переход от изомонодромных потоков к изоспектральным, как описано в п. 4.5:

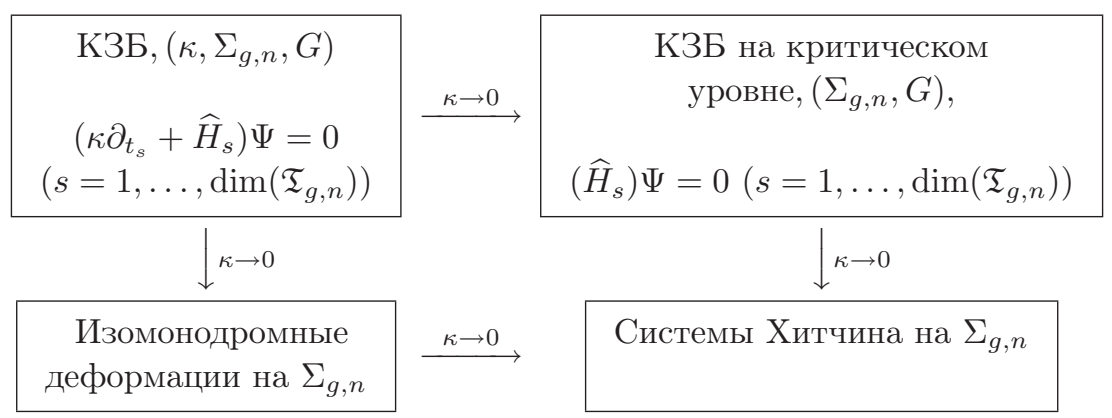

6.10. Теория поля Пенлеве. Напомним еще раз, что в предыдущих разделах мы воспроизводили изомонодромные уравнения в виде неавтономных версий механических интегрируемых систем. Более точно, в эллиптическом случае мы заменяли линейную задачу и условие совместности (уравнения Лакса)

$$
\left\{\begin{array}{l}
L(z) \Psi=\Lambda \Psi, \\
\left(\partial_{t}+M(z)\right) \Psi=0,
\end{array} \quad \partial_{t} L(z)=[L(z), M(z)],\right.
$$

на другую линейную задачу и условия совместности (изомонодромные уравнения)

$$
\left\{\begin{array}{l}
\left(\partial_{z}+L(z)\right) \Psi=0, \\
\left(\partial_{\tau}+M(z)\right) \Psi=0,
\end{array} \quad \partial_{\tau} L(z)-\partial_{z} M(z)=[L(z), M(z)] .\right.
$$

В то же время хорошо известно, что механические системы можно "обобщать" до $(1+1)$-интегрируемых теорий поля, описываемых следующими линейными задачами и условием совместности (уравнения Захарова-Шабата):

$$
\left\{\begin{array}{l}
\left(\partial_{x}+\widetilde{L}(z)\right) \Psi=0, \\
\left(\partial_{t}+\widetilde{M}(z)\right) \Psi=0,
\end{array} \quad \partial_{t} \widetilde{L}(z)-\partial_{x} \widetilde{M}(z)=[\widetilde{L}(z), \widetilde{M}(z)]\right.
$$

где фазовое пространство состоит из $\mathbb{S}^{1}$-значных полей, а $x$ - координата на единичной окружности $\mathbb{S}^{1}$. Для систем Хитчина переход $(6.57) \rightarrow(6.59)$ был описан в [61] (см. также [105], [106]).

В [107] мы предложили теоретико-полевое обобщение изомонодромных уравнений. Оно возникает из линейной задачи

$$
\left\{\begin{array}{l}
\left(\partial_{z}+\partial_{x}+L^{\prime}(z)\right) \Psi=0 \\
\left(\partial_{\tau}+M^{\prime}(z)\right) \Psi=0
\end{array}\right.
$$

с условием совместности

$$
\partial_{\tau} L^{\prime}(z)-\partial_{x} M^{\prime}(z)-\partial_{z} M^{\prime}(z)=\left[L^{\prime}(z), M^{\prime}(z)\right] .
$$


В частности, полевое обобщение уравнения Пенлеве VI выглядит следующим образом. Рассмотрим четыре трехмерных векторных поля $\mathbf{S}_{b}^{\alpha}(x), \alpha \in\left(\mathbb{Z}_{2} \times\right.$ $\left.\mathbb{Z}_{2}\right) \backslash(0,0), b \in \mathbb{Z}_{2} \times \mathbb{Z}_{2}$, и пусть $\mathbf{J}^{\mathrm{I}}\left(\beta, \partial_{x}, \tau\right), \mathbf{J}^{\mathrm{II}}\left(\beta, b, c, \partial_{x}, \tau\right)$ - псевдодифференциальные операторы

$\mathbf{J}^{\mathrm{I}}\left(\alpha, b, c, \partial_{x}, \tau\right)=E_{2}\left(\omega_{\alpha}-\frac{\bar{k}}{2 \pi i} \partial_{x}, \tau\right), \quad \mathbf{J}^{\mathrm{II}}\left(\alpha, b, c, \partial_{x}, \tau\right)=f_{\alpha}\left(\frac{\bar{k}}{2 \pi i} \partial_{x}, \omega_{c}-\omega_{b}\right)$.

Тогда полевое уравнение имеет вид четырех взаимодействующих неавтономных волчков Эйлера-Арнольда [108], [109], отвечающих группе петель $L(\operatorname{SL}(2, \mathbb{C}))$ :

$$
\begin{aligned}
\frac{\partial}{\partial \tau} \mathbf{S}_{b}^{\alpha}(x)= & \sum_{\beta \neq \alpha}\left(\mathbf{S}_{b}^{\alpha-\beta}(x) \mathbf{J}^{\mathrm{I}}\left(\alpha, \beta, \partial_{x}, \tau\right) \mathbf{S}_{b}^{\beta}(x)\right. \\
& \left.+\sum_{c \neq b} \mathbf{S}_{b}^{\alpha-\beta}(x) \mathbf{J}^{\mathrm{II}}\left(\alpha, \beta, b, c, \partial_{x}, \tau\right) \mathbf{S}_{c}^{\beta}(x)\right)
\end{aligned}
$$

с условиями $\mathbf{S}_{b}^{\alpha}(x)=(-1)^{b \times \alpha} \mathbf{S}_{b}^{\alpha}(-x)$. Уравнение (6.63) является полевым обобщением эллиптической системы Шлезингера [42], а в частном случае $\mathrm{sl}_{2}$-связности, четырех отмеченных точек в полупериодах и нуле, а также на уравнениях связей, оно становится обобщением Пенлеве VI. Можно показать, что динамика нулевых мод (6.63) на связях описывается неавтономным гиростатом Жуковского-Вольтерра (8.6) на три переменные $S^{\alpha}$ - нулевые моды $S_{0}^{\alpha}$. Как увидим далее, это одна из возможных форм записи уравнения Пенлеве VI (см. раздел 8).

\section{7. Симплектическое соответствие Гекке}

Рассмотрим преобразование Гекке в случае $g=1, n=1$. Фазовое простран$\operatorname{rank} G$

ство имеет размерность коприсоединенной орбиты $2 \sum_{j=1}\left(d_{j}-1\right)$. Оператор $L$ удовлетворяет условиям

$$
L(z+1)=\mathscr{Q} L(z) \mathscr{Q}^{-1}, \quad L(z+\tau)=\Lambda L(z) \Lambda^{-1},
$$

где $\mathscr{Q}$ и $\Lambda$ - решения (5.4) и

$$
\bar{\partial} L(z)=\mathbf{S} \delta(z, \bar{z})
$$

Другими словами, $\operatorname{Res}_{z=0} L(z)=\mathrm{S}$. Эти условия фиксируют $L$. Чтобы подчеркнуть зависимость от характеристического класса $\zeta\left(E_{G}\right)$, будем писать $L^{\varpi_{j}^{\vee}}$ для матрицы Лакса, построенной по квазипериодическим условиям с $\Lambda=\Lambda_{\varpi} \vee$ и $\mathscr{Q}_{\varpi_{j}^{\vee}}$, которые являются решениями уравнения $(5.4)$ с $\zeta=\mathbf{e}\left(-\varpi_{j}^{\vee}\right), \varpi_{j}^{\vee} \in P^{\vee}$.

Модификация $\Xi(\gamma)$ расслоения $E_{G}$ меняет характеристический класс. Ее действие на $L^{\varpi}$ имеет вид

$$
L^{\varpi_{j}^{\vee}} \Xi(\gamma)=\Xi(\gamma) L^{\varpi_{j}^{\vee}+\gamma}
$$


Это и есть упомянутое во введении сингулярное калибровочное преобразование. Действие (7.3) позволяет выписать условия на $\Xi(\gamma)$. Так как $L_{j}^{\vee}$ имеет простой полюс в $z=0$, то модифицированная матрица Лакса $L^{\varpi}{ }_{j}^{\vee}+\gamma$ также имеет простой полюс в $z=0$. Разложим $L^{\varpi}$ и $L^{\varpi_{j}^{\vee}+\gamma}$ в базисе Шевалле (А.15), (В.2):

$$
L^{\varpi_{j}^{\vee}}=L_{\mathfrak{h}}(z)+\sum_{\alpha \in R} L_{\alpha}(z) E_{\alpha}, \quad L^{\varpi_{j}^{\vee}+\gamma}=\widetilde{L}_{\mathfrak{h}}(z)+\sum_{\alpha \in R} \widetilde{L}_{\alpha}(z) E_{\alpha} .
$$

В базисе простых корней (А.2) имеем разложение $\alpha=\sum_{j=1}^{l} f_{j}^{\alpha} \alpha_{j}$, в базисе фундаментальных ковесов - разложение $\gamma=\sum_{j=1}^{l} m_{j} \varpi_{j}^{\vee}$. Предположим, что $\left\langle\gamma, \alpha_{j}\right\rangle \geqslant 0$ для простого $\alpha_{j}$. Другими словами, $\gamma$ - доминантный ковес. Тогда $\langle\gamma, \alpha\rangle=$ $\sum_{j=1}^{l} m_{j} n_{j}^{\alpha}-$ целое число. Оно положительно для $\alpha \in R^{+}$и отрицательно для $\alpha \in R^{-}$. Из (7.3) находим

$$
L_{\mathfrak{h}}^{\varpi_{j}^{\vee}+\gamma}(z)=L_{\mathfrak{h}}^{\varpi_{j}^{\vee}}(z), \quad L_{\alpha}^{\varpi_{j}^{\vee}+\gamma}(z)=z^{\langle\gamma, \alpha\rangle} L_{\alpha}^{\varpi_{j}^{\vee}}(z) .
$$

В окрестности точки $z=0$ оператор $L_{\alpha}(z)$ имеет вид

$$
L_{\alpha}^{\varpi_{j}^{\vee}}(z)=a_{\langle\gamma, \alpha\rangle} z^{-\langle\gamma, \alpha\rangle}+a_{\langle\gamma, \alpha\rangle+1} z^{-\langle\gamma, \alpha\rangle+1}+\cdots \quad\left(\alpha \in R^{-}\right),
$$

а в противном случае преобразованный оператор Лакса становится сингулярным. Это означает, что тип модификации $\gamma$ не является произвольным, но зависит от локального поведения оператора Лакса. Это позволяет (в принципе) найти размерность пространства преобразований Гекке.

Рассмотрим теперь глобальное поведение $L(z)(7.1)$. Тогда находим, что $\Xi(\gamma)$ сплетает квазипериодические условия

$$
\Xi(\gamma, z+1) \mathscr{Q}_{\varpi_{j}^{\vee}}=\mathscr{Q}_{\varpi_{j}^{\vee}+\gamma} \Xi(\gamma, z), \quad \Xi(\gamma, z+\tau) \Lambda_{\varpi_{j}^{\vee}}=\Lambda_{\varpi_{j}^{\vee}+\gamma} \Xi(\gamma, z) .
$$

Для $G=\mathrm{SL}(N, \mathbb{C}), \gamma=\varpi_{1}^{\vee}$ и специальных вычетов $L(z)$ решения этих уравнений были найдены в [61]. На редуцированном пространстве $\mathbf{P}^{\text {red }}$ уравнения движения, отвечающие гамильтонианам $I_{j k}$, имеют форму Лакса

$$
\kappa \partial_{t_{s}} L-\kappa \partial_{z} M_{t_{s}}+\left[M_{t_{s}}, L\right]=0 .
$$

Операторы $M_{t_{s}}$ строятся по $L$ - и $r$-матрицам.

Пример. Продемонстрируем процедуру модификации на примере группы $\operatorname{SL}(N, \mathbb{C})$. Характеристические классы соответствующих расслоений - элементы $\mathbb{Z}_{N}$. Значения 1 и $\exp (2 \pi i / N)$ отвечают наиболее различным расслоениям и динамическим системам. Это эллиптическая система Калоджеро и эллиптический волчок [61]. В изомонодромном случае обе модели становятся неавтономными [42], [43], [110]. При этом вследствие соответствия Пенлеве-Калоджеро 
пары Лакса остаются теми же, что и в автономной интегрируемой механике. Матрицы Лакса фиксируются вычетами в $z=0$ на $\Sigma_{\tau}$ :

$$
\begin{aligned}
& \operatorname{Res}_{z=0} L^{\mathrm{Cal}}(z)=\nu\left(\begin{array}{ccccc}
0 & 1 & 1 & \ldots & 1 \\
1 & 0 & 1 & \ldots & 1 \\
\ldots & \ldots & \ldots & \ldots \\
1 & 1 & \ldots & 1 & 0
\end{array}\right), \\
& \operatorname{Res}_{z=0} L^{\mathrm{top}}(z)=S=\sum_{m, n} T_{m n} S_{m n} \\
& \operatorname{Spec}(S)=\operatorname{diag}(\nu, \ldots, \nu,-(N-1) \nu),
\end{aligned}
$$

и граничными условиями

$$
\begin{array}{ll}
L^{\mathrm{Cal}}(z+1)=L^{\mathrm{Cal}}(z), & L^{\mathrm{top}}(z+1)=Q L^{\mathrm{top}}(z) Q^{-1}, \\
L^{\mathrm{Cal}}(z+\tau)=\mathbf{e}(-\mathbf{u}) L^{\mathrm{Cal}}(z) \mathbf{e}(\mathbf{u}), & L^{\mathrm{top}}(z+\tau)=\Lambda L^{\mathrm{top}}(z) \Lambda^{-1},
\end{array}
$$

где

$$
\mathbf{u}_{i j}=\delta_{i j} u_{i}, \quad \Lambda_{i j}=\delta^{\left(\bmod _{N}(i+1), j\right)}, \quad Q_{i j}=\delta_{i j} \mathbf{e}\left(\frac{i}{N}\right),
$$

и

$$
\left\{T_{m n}=\mathbf{e}\left(\frac{m n}{2 N}\right) Q^{m} \Lambda^{n}\right\}, \quad m, n \in \mathbb{Z}_{N},
$$

- базис синус-алгебры. Матрицы Лакса

$$
\begin{aligned}
L^{\mathrm{Cal}}(z) & =\sum_{i, j=1}^{N} E_{i j}\left(\delta_{i j} v_{i}+\nu\left(1-\delta_{i j}\right) \Phi\left(z, u_{i}-u_{j}\right)\right), \quad\left(E_{i j}\right)_{a b}=\delta_{i a} \delta_{j b}, \\
L^{\mathrm{top}}(z) & =\sum_{n^{2}+m^{2} \neq 0} S_{m n} T_{m n} \varphi_{m n}(z)
\end{aligned}
$$

генерируют функции Гамильтона

$$
\begin{aligned}
H^{\text {Cal }} & =\frac{1}{2} \sum_{k=1}^{N} v_{k}^{2}-\sum_{i<j} \nu^{2} \wp\left(u_{i}-u_{j}\right), \\
H^{\mathrm{top}} & \left.=\frac{1}{2} \sum_{n^{2}+m^{2} \neq 0} S_{m n} S_{-m,-n \wp(} \frac{m+n \tau}{N}\right) .
\end{aligned}
$$

Модификация дается матрицей (см. [61])

$\Xi_{i l}(z, U, \tau)=\theta\left[\begin{array}{c}\frac{i}{N}-\frac{1}{2} \\ \frac{N}{2}\end{array}\right]\left(z-N u_{l}, N \tau\right) D_{l}, \quad D_{l}=(-1)^{l} \prod_{j<k ; j, k \neq l} \vartheta^{-1}\left(u_{k}-u_{j}, \tau\right)$. 
Она действует калибровочным преобразованием и, следовательно, устанавливает следующее соотношение между системами:

$$
L^{\text {top }}(z)=\Xi(z) L^{\mathrm{Cal}}(z) \Xi^{-1}(z)-\kappa \partial_{z} \Xi(z) \Xi^{-1}(z)+\frac{\kappa}{N} E_{1}(z)
$$

Последний член можно перенести в определение $L^{\text {top }}$ или $L^{\mathrm{Cal}}$, так как он пропорционален единичной матрице.

\section{8. Пенлеве VI}

8.1. Три формы уравнения Пенлеве VI. В этом разделе мы опишем три формы уравнения Пенлеве VI, а также связи между ними и соответствующими линейными задачами. Мы имеем в виду следующие три формы.

1. Рациональная форма была найдена Б. Гамбье [3], [1], [14], [15] как последнее и наиболее общее уравнение в списке обыкновенных дифференциальных уравнений второго порядка, удовлетворяющих свойству Пенлеве:

$$
\begin{gathered}
\frac{d^{2} X}{d T^{2}}=\frac{1}{2}\left(\frac{1}{X}+\frac{1}{X-1}+\frac{1}{X-T}\right)\left(\frac{d X}{d T}\right)^{2}-\left(\frac{1}{T}+\frac{1}{T-1}+\frac{1}{X-T}\right) \frac{d X}{d T} \\
+\frac{X(X-1)(X-T)}{T^{2}(T-1)^{2}}\left(\alpha+\beta \frac{T}{X^{2}}+\gamma \frac{T-1}{(X-1)^{2}}+\delta \frac{T(T-1)}{(X-T)^{2}}\right) .
\end{gathered}
$$

Оно содержит четыре свободных комплексных константы $\alpha, \beta, \gamma, \delta$.

2. Эллиптическая форма была найдена П. Пенлеве [62], [63]:

$$
\frac{d^{2} u}{d \tau^{2}}=\sum_{a=0}^{3} \nu_{a}^{2} \wp^{\prime}\left(u+\omega_{a}\right), \quad\left\{\omega_{a}, k=0, \ldots, 3\right\}=\left\{0, \frac{1}{2}, \frac{1+\tau}{2}, \frac{\tau}{2}\right\} .
$$

Уравнение задано на эллиптической кривой $\Sigma_{\tau}$ с полупериодами $\omega_{a}$ и модулем $\tau$, который является здесь временно́й переменной. Связь между (8.1) и (8.2) дается заменой переменных

$$
\begin{gathered}
(u, \tau) \rightarrow\left(X(u, \tau)=\frac{\wp(u)-e_{1}}{e_{2}-e_{1}}, T(\tau)=\frac{e_{3}-e_{1}}{e_{2}-e_{1}}\right), \quad e_{\alpha} \equiv \wp\left(\omega_{\alpha}\right), \\
\left(\nu_{0}^{2}, \nu_{1}^{2}, \nu_{2}^{2}, \nu_{3}^{2}\right)=-4 \pi^{2}\left(\alpha,-\beta, \gamma,-\delta+\frac{1}{2}\right) .
\end{gathered}
$$

В таком виде уравнение очевидно является гамильтоновым. Механическая модель описывается гамильтонианом

$$
H^{\mathrm{P} \mathrm{VI}}=\frac{1}{2} v^{2}-\sum_{a=0}^{3} \nu_{a}^{2} \wp\left(u+\omega_{a}\right)
$$

и каноническими скобками Пуассона

$$
\{v, u\}=1
$$

Механика неавтономна, так как два полупериода и ю-функция Вейерштрасса явно зависят от $\tau$. 
3. Неавтономныц гиростат Жуковского-Вольтерра был определен в [64]. Там же была доказана его эквивалентность уравнению Пенлеве VI. Эта форма имеет вид

$$
\partial_{\tau} S=[S, J(S)]+\left[S, \nu^{\prime}\right]
$$

где $S$ суть $\mathrm{sl}_{2}^{*}$-значные динамические переменные. В базисе матриц Паули

$$
\begin{gathered}
S=\sum_{\alpha=1}^{3} S_{\alpha} \sigma_{\alpha}, \quad J(S)=\sum_{\alpha=1}^{3} J_{\alpha}(\tau) S_{\alpha} \sigma_{\alpha}, \\
J_{\alpha}(\tau)=\wp\left(\omega_{\alpha}\right)=e_{\alpha}, \quad \alpha=1,2,3, \\
\nu^{\prime}=\sum_{\alpha=1}^{3} \nu_{\alpha}^{\prime} \sigma_{\alpha}, \quad \nu_{a}^{\prime}=-\widetilde{\nu}_{a} \exp \left(-2 \pi i \omega_{a} \partial_{\tau} \omega_{a}\right)\left(\frac{\vartheta^{\prime}(0)}{\vartheta\left(\omega_{a}\right)}\right)^{2}, \quad a=0,1,2,3,
\end{gathered}
$$

где $\widetilde{\nu}_{a}$ - четыре комплексные константы. Автономная версия уравнения (8.6) (когда время не связано с модулем $\tau$ ) известна как гиростат Жуковского-Вольтерра [111], [112]. Вектор $\left(\nu_{1}^{\prime}, \nu_{2}^{\prime}, \nu_{3}^{\prime}\right)$ играет роль гиростатического момента, а $J_{\alpha}(\tau)$ - обратные компоненты тензора инерции в главных осях.

Уравнение (8.6) гамильтоново с гамильтонианом

$$
H^{\mathrm{ZVG}}=\frac{1}{2} \sum_{\alpha=1}^{3}\left(J_{\alpha} S_{\alpha}^{2}+S_{\alpha} \nu_{\alpha}^{\prime}\right)
$$

и скобками Пуассона-Ли на $\mathrm{sl}_{2}^{*}$ :

$$
\left\{S_{\alpha}, S_{\beta}\right\}=\varepsilon_{\alpha \beta \gamma} S_{\gamma}
$$

Связь между (8.6) и (8.2) дается заменой переменных

$$
\begin{aligned}
S_{1}= & -v \frac{\theta_{2}(0)}{\vartheta^{\prime}(0)} \frac{\theta_{2}(2 u)}{\vartheta(2 u)}-\frac{\kappa}{2} \frac{\theta_{2}(0)}{\vartheta^{\prime}(0)} \frac{\theta_{2}^{\prime}(2 u)}{\vartheta(2 u)}+\widetilde{\nu}_{0} \frac{\theta_{2}^{2}(0)}{\theta_{3}(0) \theta_{4}(0)} \frac{\theta_{3}(2 u) \theta_{4}(2 u)}{\vartheta^{2}(2 u)} \\
& +\widetilde{\nu}_{1} \frac{\theta_{2}^{2}(2 u)}{\vartheta^{2}(2 u)}+\widetilde{\nu}_{2} \frac{\theta_{2}(0)}{\theta_{4}(0)} \frac{\theta_{2}(2 u) \theta_{4}(2 u)}{\vartheta^{2}(2 u)}+\widetilde{\nu}_{3} \frac{\theta_{2}(0)}{\theta_{3}(0)} \frac{\theta_{2}(2 u) \theta_{3}(2 u)}{\vartheta^{2}(2 u)}, \\
i S_{2}= & v \frac{\theta_{3}(0)}{\vartheta^{\prime}(0)} \frac{\theta_{3}(2 u)}{\vartheta(2 u)}+\frac{\kappa}{2} \frac{\theta_{3}(0)}{\vartheta^{\prime}(0)} \frac{\theta_{3}^{\prime}(2 u)}{\vartheta(2 u)}-\widetilde{\nu}_{0} \frac{\theta_{3}^{2}(0)}{\theta_{2}(0) \theta_{4}(0)} \frac{\theta_{2}(2 u) \theta_{4}(2 u)}{\vartheta^{2}(2 u)} \\
& -\widetilde{\nu}_{1} \frac{\theta_{3}(0)}{\theta_{2}(0)} \frac{\theta_{3}(2 u) \theta_{2}(2 u)}{\vartheta^{2}(2 u)}-\widetilde{\nu}_{2} \frac{\theta_{3}(0)}{\theta_{4}(0)} \frac{\theta_{3}(2 u) \theta_{4}(2 u)}{\vartheta^{2}(2 u)}-\widetilde{\nu}_{3} \frac{\theta_{3}^{2}(2 u)}{\vartheta^{2}(2 u)}, \\
S_{3}= & -v \frac{\theta_{4}(0)}{\vartheta^{\prime}(0)} \frac{\theta_{4}(2 u)}{\vartheta(2 u)}-\frac{\kappa}{2} \frac{\theta_{4}(0)}{\vartheta^{\prime}(0)} \frac{\theta_{4}^{\prime}(2 u)}{\vartheta(2 u)}+\widetilde{\nu}_{0} \frac{\theta_{4}^{2}(0)}{\theta_{2}(0) \theta_{3}(0)} \frac{\theta_{2}(2 u) \theta_{3}(2 u)}{\vartheta^{2}(2 u)} \\
& +\widetilde{\nu}_{1} \frac{\theta_{4}(0)}{\theta_{2}(0)} \frac{\theta_{2}(2 u) \theta_{4}(2 u)}{\vartheta^{2}(2 u)}+\widetilde{\nu}_{2} \frac{\theta_{4}^{2}(2 u)}{\vartheta^{2}(2 u)}+\widetilde{\nu}_{3} \frac{\theta_{4}(0)}{\theta_{3}(0)} \frac{\theta_{4}(2 u) \theta_{3}(2 u)}{\vartheta^{2}(2 u)}
\end{aligned}
$$


и следующим соотношением на константы:

$$
\begin{aligned}
\widetilde{\nu}_{0} & =\frac{1}{2}\left(\nu_{0}+\nu_{1}+\nu_{2}+\nu_{3}\right), \\
\widetilde{\nu}_{1} & =\frac{1}{2}\left(\nu_{0}+\nu_{1}-\nu_{2}-\nu_{3}\right), \\
\widetilde{\nu}_{2} & =\frac{1}{2}\left(\nu_{0}-\nu_{1}+\nu_{2}-\nu_{3}\right), \\
\widetilde{\nu}_{3} & =\frac{1}{2}\left(\nu_{0}-\nu_{1}-\nu_{2}+\nu_{3}\right) .
\end{aligned}
$$

Заметим, что три константы $\left(\widetilde{\nu}_{1}, \widetilde{\nu}_{2}, \widetilde{\nu}_{3}\right)$ входят в уравнения (8.6) явно, а последняя $\nu_{0}^{\prime}$ связана со значением функции Казимира скобок (8.9):

$$
\frac{1}{2} \sum_{\alpha=1}^{3} S_{\alpha}^{2}=\widetilde{\nu}_{0}^{2}=\nu_{0}^{\prime 2}
$$

т. е. три линейные комбинации четырех констант из уравнения Пенлеве VI (8.2) объединяются в вектор гиростатического момента, а последняя есть длина углового момента.

8.2. Линейные задачи. Опишем линейные задачи для приведенных выше трех форм уравнения Пенлеве VI.

1. Рациональная форма. Линейная задача естественно возникает из $\mathrm{sl}_{2}$-системы Шлезингера [6], [9]-[11] на $\mathbb{C P}^{1} \backslash\{0,1, T, \infty\}$. Она описывает поведение связности

$$
\left(\partial_{\zeta}+A(\zeta)\right) d \zeta=\left(\partial_{\zeta}+\frac{A^{0}}{\zeta}+\frac{A^{1}}{\zeta-1}+\frac{A^{T}}{\zeta-T}\right) d \zeta
$$

с логарифмическими особенностями в $\{0,1, T, \infty\}$ и $\mathrm{sl}_{2}^{*}$-значными вычетами. Изомонодромное уравнение есть условие совместности линейной задачи

$$
\left\{\begin{array}{l}
\left(\partial_{\zeta}+A(\zeta)\right) \Psi=0, \\
\left(\partial_{T}+M(\zeta) \Psi\right)=0,
\end{array} \quad M(\zeta)=-\frac{A^{T}}{\zeta-T},\right.
$$

и имеет вид

$$
\partial_{T} A(\zeta)-\partial_{\zeta} M(\zeta)=[A(\zeta), M(\zeta)]
$$

Для получения Пенлеве VI нужно выполнить редукцию

$$
\mathscr{O}^{0} \times \mathscr{O}^{1} \times \mathscr{O}^{T} \times \mathscr{O}^{\infty} \longrightarrow \mathscr{O}^{0} \times \mathscr{O}^{1} \times \mathscr{O}^{T} \times \mathscr{O}^{\infty} / / \mathrm{SL}(2, \mathbb{C}),
$$

где $\mathscr{O}^{a}$ - коприсоединенная орбита, т. е. коалгебра $A^{a}$ с фиксированными собственными значениями, которые являются свободными константами. Редукция (8.16) генерируется глобальным ( $\zeta$-независимым) коприсоединенным действием $A^{c} \rightarrow \operatorname{Ad}_{\mathrm{SL}(2, \mathbb{C})}^{*} A^{c}, c=0,1, T, \infty$. Это дает уравнение момента

$$
A^{0}+A^{1}+A^{T}+A^{\infty}=0 .
$$

На редуцированном пространстве нужно еще выбрать координаты. Для случая $(2 \times 2)$-пар Лакса рецепт выбора канонических переменных хорошо известен: пусть $A_{12}(X)=0$ и $P=A_{11}(X)$, тогда $\{P, X\}=1$ (см., например, [113]). После довольно длинных вычислений получим (8.1). 
2. Эллиптическая форма. Линейная $(2 \times 2)$-задача была предложена в [65]. Она формулируется в терминах связности $\left(\partial_{z}+L^{\mathrm{P}} \mathrm{vI}(z)\right) d z$ в голоморфном расслоении на эллиптической кривой с функциями переклейки $g_{1}=\left(\begin{array}{ll}1 & 0 \\ 0 & 1\end{array}\right)$ и $g_{\tau}=\left(\begin{array}{cc}\mathbf{e}(u) & 0 \\ 0 & \mathbf{e}(-u)\end{array}\right)$ :

$$
\begin{aligned}
L^{\mathrm{P}_{\mathrm{VI}}}(z) & =\left(\begin{array}{cc}
v & 0 \\
0 & -v
\end{array}\right)+\sum_{c=0}^{3} L_{c}^{\mathrm{P} \mathrm{VI}}, \\
L_{c}^{\mathrm{PVI}} & =\widetilde{\nu}_{c}\left(\begin{array}{cc}
0 & \varphi_{c}\left(2 u, z+\omega_{c}\right) \\
\varphi_{c}\left(-2 u, z+\omega_{c}\right) & 0
\end{array}\right),
\end{aligned}
$$

где $\widetilde{\nu}_{c}$ определены в (8.11). Вместе с $M$-оператором

$$
M^{\mathrm{PVI}}(z)=\sum_{c=0}^{3} M_{c}^{\mathrm{PVI}}, \quad M_{c}^{\mathrm{PVI}}=\widetilde{\nu}_{c}\left(\begin{array}{cc}
0 & f_{c}\left(2 u, z+\omega_{c}\right) \\
f_{c}\left(-2 u, z+\omega_{c}\right) & 0
\end{array}\right)
$$

матрица Лакса (8.18) дает уравнения Пенлеве VI (8.2)

$$
\partial_{\tau} L^{\mathrm{PVI}}(z)-\partial_{z} M^{\mathrm{PVI}}(z)=\frac{1}{\kappa}\left[L^{\mathrm{PVI}}(z), M^{\mathrm{PVI}}(z)\right]
$$

Заметим, что матрица Лакса $(8.18)$ - сечение расслоения над $\Sigma_{\tau}=\mathbb{C} / \mathbb{Z}+\mathbb{Z} \tau$. Уравнение Пенлеве VI можно получить и иначе - из следующей пары Лакса:

$$
\begin{aligned}
\widetilde{L}^{\mathrm{PVI}}(z) & =\left(\begin{array}{cc}
v & 0 \\
0 & -v
\end{array}\right)+\sum_{c=0}^{3} \widetilde{L}_{c}^{\mathrm{PVI}}, \\
\widetilde{L}_{c}^{\mathrm{PVI}} & =\nu_{c}\left(\begin{array}{cc}
0 & \varphi_{c}\left(z, \omega_{c}+u\right) \\
\varphi_{c}\left(z, \omega_{c}-u\right) & 0
\end{array}\right), \\
\widetilde{M}^{\mathrm{PVI}}(z) & =\sum_{c=0}^{3} \widetilde{M}_{c}^{\mathrm{PVI}}, \\
\widetilde{M}_{c}^{\mathrm{PVI}} & =\nu_{c}\left(\begin{array}{cc}
0 & f_{c}\left(z, \omega_{c}+u\right) \\
f_{c}\left(z, \omega_{c}-u\right) & 0
\end{array}\right),
\end{aligned}
$$

с $\nu_{c}$ из (8.2). Здесь (8.21) - сечение голоморфного расслоения над удвоенной эллиптической кривой $\Sigma_{2,2 \tau}=\mathbb{C} / 2 \mathbb{Z}+2 \mathbb{Z} \tau$.

3. Неавтономный гиростат Жуковского-Вольтерра. Линейная задача со спектральным параметром на эллиптической кривой была предложена в [64]. Аналогично предыдущему случаю имеем связность $\left(\partial_{z}+L^{\mathrm{ZVG}}(z)\right) d z$ в голоморфном расслоении на эллиптической кривой $\Sigma_{\tau}=\mathbb{C} / \mathbb{Z}+\mathbb{Z} \tau$ с функциями пе- 


$$
\begin{aligned}
\text { реклейки }^{7} g_{1}= & -Q=\left(\begin{array}{cc}
1 & 0 \\
0 & -1
\end{array}\right) \text { и } g_{\tau}=-\mathbf{e}\left(-\frac{\tau}{4}-\frac{z}{2}\right) \Lambda=-\mathbf{e}\left(-\frac{\tau}{4}-\frac{z}{2}\right)\left(\begin{array}{cc}
0 & 1 \\
1 & 0
\end{array}\right): \\
L^{\mathrm{ZVG}}(z)= & -\frac{\kappa}{2} \partial_{z} \log (\vartheta(z ; \tau)) \sigma_{0}+\sum_{\alpha=1}^{3}\left(S_{\alpha} \varphi_{\alpha}(z)+\nu_{\alpha} \varphi_{\alpha}\left(z-\omega_{\alpha}\right)\right) \sigma_{\alpha}, \\
M^{\mathrm{ZVG}}(z)= & -\frac{\kappa}{2} \partial_{\tau} \log (\vartheta(z ; \tau)) \sigma_{0}-\sum_{\alpha=1}^{3} S_{\alpha} \frac{\varphi_{1}(z) \varphi_{2}(z) \varphi_{3}(z)}{\varphi_{\alpha}(z)} \sigma_{\alpha} \\
& +E_{1}(z) L^{\mathrm{ZVG}}(\kappa=0) .
\end{aligned}
$$

Изомонодромные уравнения

$$
\partial_{\tau} L^{\mathrm{ZVG}}(z)-\partial_{z} M^{\mathrm{ZVG}}(z)=\frac{1}{\kappa}\left[L^{\mathrm{ZVG}}(z), M^{\mathrm{ZVG}}(z)\right]
$$

эквивалентны (8.6).

Тригонометрические и рациональные вырождения пар Лакса для систем типа Калоджеро-Годена-Шлезингера исследовались в [114]-[117]. Еще одна эллиптическая $(2 \times 2)$-пара Лакса для уравнения Пенлеве VI возникает в квантовой версии соответствия Калоджеро-Пенлеве [95], [96]. Матричный элемент $L_{12}(z)$ имеет два простых нуля в $\pm u$ и не имеет полюсов.

8.3. Эллиптическая форма рациональной связности. Для установления связи между рациональной и эллиптической связностями необходимо поднять расслоение на $\mathbb{C P}^{1}$ до расслоения на эллиптической кривой. Такой способ был предложен в [118]. Выполним для (8.13) подстановку, аналогичную (8.3), т. е. сделаем замену переменных для спектрального параметра в рациональной связности $A(\zeta)=\left(\frac{A^{0}}{\zeta}+\frac{A^{1}}{\zeta-1}+\frac{A^{T}}{\zeta-T}\right) d \zeta$ :

$$
\zeta=\zeta(z, \tau)=\frac{\wp(z / 2)-e_{1}}{e_{2}-e_{1}}, \quad T=T(\tau)=\frac{e_{3}-e_{1}}{e_{2}-e_{1}} .
$$

Это дает

$$
\begin{aligned}
A(\zeta) d \zeta & =\sum_{\gamma=0,1, T} \frac{A^{\gamma} \wp^{\prime}(z / 2)}{\wp(z / 2)-\wp\left(\omega^{\gamma}\right)} \frac{d z}{2} \\
& =\sum_{\gamma=0,1, T} A^{\gamma}\left(E_{1}\left(\frac{z}{2}+\omega^{\gamma}\right)-E_{1}\left(\frac{z}{2}\right)-E_{1}\left(\omega^{\gamma}\right)\right) d z
\end{aligned}
$$

где полупериоды $\omega^{0}, \omega^{1}, \omega^{T}$ кривой $\Sigma_{\tau}$ связаны с обозначениями $\omega_{a}$ из $(8.2)$ так:

$$
\omega^{0}=\omega_{1}=\frac{1}{2}, \quad \omega^{1}=\omega_{2}=\frac{1+\tau}{2}, \quad \omega^{T}=\omega_{3}=\frac{\tau}{2} .
$$

Заметим, что

$$
E_{1}\left(\frac{z}{2}+\omega_{\gamma}\right)-E_{1}\left(\frac{z}{2}\right)-E_{1}\left(\omega_{\gamma}\right)=-\varphi_{\alpha}(z)-\varphi_{\beta}(z)
$$

\footnotetext{
${ }^{7}$ Скалярные множители не очень важны, так как $\partial_{z}+L(z)-$ связность в присоединенном расслоении.
} 
для любых различных индексов $\alpha, \beta, \gamma$ из $\{1,2,3\}$. Действительно, обе части (8.28) - двоякопериодические функции на удвоенной эллиптической кривой $\Sigma_{2,2 \tau}$ с простыми полюсами в ее полупериодах $(0,1, \tau+1, \tau)$. Остается лишь сравнить вычеты. Следовательно, (8.27) можно переписать в виде

$$
A(\zeta) d \zeta=\sum_{\alpha=1}^{3} B^{\alpha} \varphi_{\alpha}(z) d z
$$

где

$$
\left\{\begin{array} { l } 
{ B ^ { 1 } = - A ^ { 1 } - A ^ { T } , } \\
{ B ^ { 2 } = - A ^ { 0 } - A ^ { T } , } \\
{ B ^ { 3 } = - A ^ { 0 } - A ^ { 1 } , }
\end{array} \quad \left\{\begin{array}{l}
A^{\infty}=\frac{1}{2}\left(+B^{1}+B^{2}+B^{3}\right), \\
A^{0}=\frac{1}{2}\left(+B^{1}-B^{2}-B^{3}\right), \\
A^{1}=\frac{1}{2}\left(-B^{1}+B^{2}-B^{3}\right), \\
A^{T}=\frac{1}{2}\left(-B^{1}-B^{2}+B^{3}\right) .
\end{array}\right.\right.
$$

Выражение

$$
L^{\mathrm{ell}}(z)=\sum_{\alpha=1}^{3} B^{\alpha} \varphi_{\alpha}(z)
$$

есть двоякопериодическая функция на удвоенной эллиптической кривой $\Sigma_{2,2 \tau}$ с простыми полюсами в полупериодах $(0,1, \tau+1, \tau)$. Вычеты равны $2 A^{\infty}, 2 A^{0}$, $2 A^{1}, 2 A^{T}$ соответственно.

8.4. Симплектическое соответствие Гекке. Модификацию расслоений можно рассматривать как процедуру, связывающую расслоения с разными характеристическими классами и описывающую симплектическое соответствие Гекке [61], [45]. В случае $\mathrm{SL}(2, \mathbb{C})$ характеристические классы являются элементами из $\mathbb{Z}_{2}$, т. е. равны \pm 1 . Тривиальный класс “+1" отвечает системе $(8.18)-(8.20)$, a "-1" отвечает системе (8.23)-(8.25). Соответствие Гекке связывает эллиптические пары Лакса (8.18)-(8.20) и (8.23)-(8.25) модификацией (7.14). В случае $\operatorname{SL}(2, \mathbb{C})$ она имеет вид

$$
\Xi(z)=\left(\begin{array}{cc}
\theta_{3}(z-2 u, 2 \tau) & -\theta_{3}(z+2 u, 2 \tau) \\
-\theta_{2}(z-2 u, 2 \tau) & \theta_{2}(z+2 u, 2 \tau)
\end{array}\right) .
$$

Она отображает голоморфное расслоение для $\mathrm{P}_{\mathrm{VI}}$ в другое, отвечающее ZVG. Следовательно, связности взаимосвязаны в следующем смысле:

$$
L^{\mathrm{ZVG}}(z)=\Xi(z) L^{\mathrm{PVI}_{\mathrm{VI}}}(z) \Xi^{-1}(z)-\kappa \partial_{z} \Xi(z) \Xi^{-1}(z) .
$$

Это соотношение дает замену переменных (8.10) (см. [64]).

Аналогично, модификация (8.32) связывает (8.21) и (8.31) на кривой $\Sigma_{2,2 \tau}$. Связность (8.21) имеет нетривиальный автоморфизм: сопряжение с помощью $\left(\begin{array}{ll}0 & 1 \\ 1 & 0\end{array}\right)$, когда $z \rightarrow-z$ (это отвечает смене ветви эллиптической кривой, реализованной как двулистное накрытие $\mathbb{C P}^{1}$, разветвленное в четырех точках). 
Можно проверить, что модификация (8.32) тривиализует этот автоморфизм. В итоге имеем

$$
L^{\mathrm{ell}}(z)-\frac{\kappa}{2} E_{1}(z) \sigma_{0}=\Xi(z) \widetilde{L}^{\mathrm{PVI}}(z) \Xi^{-1}(z)-\kappa \partial_{z} \Xi(z) \Xi^{-1}(z) .
$$

Это соотношение задает нетривиальную параметризацию пространства $\mathscr{O}^{0} \times$ $\mathscr{O}^{1} \times \mathscr{O}^{T} \times \mathscr{O}^{\infty} / / \mathrm{SL}(2, \mathbb{C})$ в канонических переменных $v, u$. Это означает, что $B^{\alpha}-$ из (8.31) и, следовательно, $A^{0}, A^{1}, A^{T}$ и $A^{\infty}$ получаются как функции $v, u$. Явные выражения были найдены в [118]. В частности,

$$
\begin{aligned}
& \left.B^{1}\right|_{\kappa=0}= \\
& \quad=\left(\begin{array}{cc}
-v \frac{\theta_{2}(0) \theta_{2}(u)}{\vartheta^{\prime}(0) \vartheta(u)}-\nu_{1} \frac{\theta_{2}^{2}(0)}{\theta_{3}(0) \theta_{4}(0)} \frac{\theta_{3}(u) \theta_{4}(u)}{\vartheta^{2}(u)} & \nu_{3} \frac{\theta_{2}(0) \theta_{2}(u)}{\theta_{3}(0) \theta_{3}(u)}-\nu_{4} \frac{\theta_{2}(0) \theta_{2}(u)}{\theta_{4}(0) \theta_{4}(u)} \\
\nu_{3} \frac{\theta_{2}(0) \theta_{2}(u)}{\theta_{3}(0) \theta_{3}(u)}+\nu_{4} \frac{\theta_{2}(0) \theta_{2}(u)}{\theta_{4}(0) \theta_{4}(u)} & v \frac{\theta_{2}(0) \theta_{2}(u)}{\vartheta^{\prime}(0) \vartheta(u)}+\nu_{1} \frac{\theta_{2}^{2}(0)}{\theta_{3}(0) \theta_{4}(0)} \frac{\theta_{3}(u) \theta_{4}(u)}{\vartheta^{2}(u)}
\end{array}\right) .
\end{aligned}
$$

\section{Приложение А. Простые группы Ли}

Большая часть собранных здесь фактов и обозначений может быть найдена в [83], [119].

Корни и веса. $V$ - векторное пространство над $\mathbb{R}, \operatorname{dim} V=n ; V^{*}-$ двойственное ему, а $\langle\cdot, \cdot\rangle$ - спаривание между $V$ и $V^{*}$.

$R=\{\alpha\}$ - система корней в $V^{*}$. Дуальная система $R^{\vee}=\left\{\alpha^{\vee}\right\}$ - система корней в $V$. Если $V$ и $V^{*}$ отождествляются скалярным произведением $(\cdot, \cdot)$, то $\alpha^{\vee}=2 \alpha /(\alpha, \alpha)$.

Группа автоморфизмов $V^{*}$, генерируемых отражениями

$$
s_{\alpha}: x \mapsto x-\langle x, \alpha\rangle \alpha^{\vee},
$$

есть группа Вейля $W(R)$.

Простые корни $\Pi=\left(\alpha_{1}, \ldots, \alpha_{l}\right)$ образуют базис в $R$ :

$$
\alpha=\sum_{j=1}^{n} f_{j}^{\alpha} \alpha_{j}, \quad f_{j}^{\alpha} \in \mathbb{Z},
$$

и $f_{j}^{\alpha}$ или все положительны (в этом случае $\alpha \in R^{+}-$положительный корень), или все отрицательны ( $\alpha$ - отрицательный корень). $R=R^{+} \cup R^{-}$. Уровень $\alpha-$ это сумма

$$
f_{\alpha}=\sum_{\alpha_{j} \in \Pi} f_{j}
$$

Матрица Картана:

$$
a_{j k}=\left\langle\alpha_{j}, \alpha_{k}^{\vee}\right\rangle, \quad \alpha_{j} \in \Pi, \quad \alpha_{k}^{\vee} \in \Pi^{\vee} .
$$

Простые корни генерируют решетку $Q=\sum_{j=1}^{n} n_{j} \alpha_{j}\left(n_{j} \in \mathbb{Z}, \alpha_{j} \in \Pi\right)$ в $V^{*}$. Существует единственный максимальный корень $-\alpha_{0} \in R^{+}$, равный

$$
-\alpha_{0}=\sum_{\alpha_{j} \in \Pi} n_{j} \alpha_{j}
$$


Его уровень равен $h-1$, где

$$
h=1+\sum_{\alpha_{j} \in \Pi} n_{j}
$$

- число Кокстера. Положительная камера Вейля:

$$
C^{+}=\left\{x \in V \mid\langle x, \alpha\rangle>0, \alpha \in R^{+}\right\} .
$$

Группа Вейля действует транзитивно на множестве камер Вейля. Множество простых кокорней $\Pi^{\vee}=\left(\alpha_{1}^{\vee}, \ldots, \alpha_{l}^{\vee}\right)$ образует базис в $V$ и генерирует решетку кокорней

$$
Q^{\vee}=\sum_{j=1}^{n} n_{j} \alpha_{j}^{\vee} \subset V, \quad n_{j} \in \mathbb{Z} .
$$

Решетка весов

$$
P=\sum_{j=1}^{n} m_{j} \varpi_{j} \subset V^{*}, \quad m_{j} \in \mathbb{Z},
$$

двойственна решетке кокорней (А.8). Фундаментальные ковеса

$$
\Upsilon^{\vee}=\left\{\varpi_{j}^{\vee} \in \mathfrak{h}, j=1, \ldots, n \mid\left\langle\alpha_{k}, \varpi_{j}^{\vee}\right\rangle=\delta_{k j}, \alpha_{j} \in \Pi\right\}
$$

двойственны простым корням. Они генерируют решетку ковесов

$$
P^{\vee}=\sum_{j=1}^{l} m_{j} \varpi_{j}^{\vee}, \quad m_{j} \in \mathbb{Z},
$$

двойственную корневой решетке $Q$. Полусумма положительных корней равна

$$
\rho=\frac{1}{2} \sum_{\alpha \in R^{+}} \alpha=\frac{1}{2} \sum_{j=1}^{n} \varpi_{j} .
$$

Для дуального вектора из $V$ имеем

$$
\rho^{\vee}=\frac{1}{2} \sum_{\alpha \in R^{\vee+}} \alpha^{\vee}=\sum_{j=1}^{n} \varpi_{j}^{\vee} .
$$

Аффинная группа Вейля. Аффинная группа Вейля $W_{a}$ есть $Q^{\vee} \rtimes W$ :

$$
W_{Q}=Q^{\vee} \rtimes W, \quad x \rightarrow x-\langle\alpha, x\rangle \alpha^{\vee}+k \beta^{\vee}, \quad \alpha^{\vee}, \beta^{\vee} \in R^{\vee}, \quad k \in \mathbb{Z} .
$$

Алъковы Вейля - это связные компоненты множества $V \backslash\{\langle\alpha, x\rangle \in \mathbb{Z}\}$. Их замыкания являются фундаментальными областями $W_{a}$-действия. Альков принадлежит $C^{+}(\mathrm{A} .7)$, т. е.

$$
C_{\text {alc }}=\left\{x \in V \mid\langle\alpha, x\rangle>0, \alpha \in \Pi,\left(\alpha_{0}, x\right)>-1\right\} .
$$


Оператор сдвига $x \rightarrow x+\gamma, \gamma \in P^{\vee}$, генерирует полупрямое произведение

$$
W_{P}=P^{\vee} \rtimes W .
$$

Факторгруппа изоморфна центру: $W_{P} / W_{Q} \sim P^{\vee} / Q^{\vee} \sim \mathscr{Z}(\bar{G})$.

Базис Шевалле в $\mathfrak{g}$. Пусть $\mathfrak{g}$ - простая алгебра Ли над $\mathbb{C}$ ранга $n$ и $\mathfrak{h}-$ подалгебра Картана. Пусть также $\mathfrak{h}=V+i V$, где $V$ - векторное пространство, заданное ранее для системы корней $R$. Алгебра $\mathfrak{g}$ имеет корневое разложение

$$
\mathfrak{g}=\mathfrak{h}+\mathfrak{l}, \quad \mathfrak{l}=\sum_{\beta \in R} \mathfrak{R}_{\beta}, \quad \operatorname{dim}_{\mathbb{C}} \mathfrak{R}_{\beta}=1 .
$$

Базис Шевалле в $\mathfrak{g}$ образован генераторами

$$
\left\{E_{\beta_{j}} \in \mathfrak{R}_{\beta_{j}}, \beta_{j} \in R ; H_{\alpha_{k}} \in \mathfrak{h}, \alpha_{k} \in \Pi\right\},
$$

где $H_{\alpha_{k}}$ задаются коммутационными соотношениями

$$
\begin{gathered}
{\left[E_{\alpha_{k}}, E_{-\alpha_{k}}\right]=H_{\alpha_{k}}, \quad\left[H_{\alpha_{k}}, E_{ \pm \alpha_{j}}\right]=a_{k j} E_{ \pm \alpha_{k}}, \quad \alpha_{k}, \alpha_{j} \in \Pi} \\
{\left[H_{\alpha_{j}}, E_{\alpha_{k}}\right]=a_{k j} E_{\alpha_{k}}, \quad\left[E_{\alpha}, E_{\beta}\right]=C_{\alpha, \beta} E_{\alpha+\beta},}
\end{gathered}
$$

где $C_{\alpha, \beta}-$ структурные константы $\mathfrak{g}$. Они обладают свойствами

$$
\begin{aligned}
C_{\alpha, \beta} & =-C_{\beta, \alpha}, \\
C_{\lambda \alpha, \beta} & =C_{\alpha, \lambda^{-1} \beta}, \quad \lambda \in W, \\
C_{\alpha+\beta,-\alpha} & =\frac{|\beta|^{2}}{|\alpha+\beta|^{2}} C_{-\alpha,-\beta} .
\end{aligned}
$$

Если $(\cdot, \cdot)$ - скалярное произведение в $\mathfrak{h}$, то $H_{\alpha}$ можно отождествить с кокорнями $H_{\alpha}=\alpha^{\vee}=2 \alpha /(\alpha, \alpha)$ и

$$
\left(H_{\alpha}, H_{\beta}\right)=\frac{4(\alpha, \beta)}{(\alpha, \alpha)(\beta, \beta)}=\frac{2}{(\alpha, \alpha)} a_{\alpha, \beta} .
$$

Форма Киллинга на подпространстве $\mathfrak{l}$ выражается в терминах $(\alpha, \alpha)$ :

$$
\left(E_{\alpha}, E_{\beta}\right)=\delta_{\alpha,-\beta} \frac{2}{(\alpha, \alpha)} .
$$

Центры простых групп. Односвязная группа $\bar{G}$ во всех случаях, кроме $G_{2}, F_{4}$ и $E_{8}$, имеет нетривиальный центр $\mathscr{Z}(\bar{G}) \sim P^{\vee} / Q^{\vee}$.

Группа $\mathscr{Z}(\bar{G})$ - циклическая, кроме $\mathfrak{g}=D_{4 l}$, и $\operatorname{ord}(\mathscr{Z}(\bar{G}))=\operatorname{det}\left(a_{k j}\right)$, где $\left(a_{k j}\right)$ - матрица Картана. Кроме того,

$$
G^{\mathrm{ad}}=\bar{G} / \mathscr{Z}(\bar{G})
$$

В случаях $A_{n-1}(n-$ составное $)$ и $D_{n}$ центр $\mathscr{Z}(\bar{G})$ имеет нетривиальные подгруппы $\mathscr{Z}_{l} \sim \mu_{l}=\mathbb{Z} / l \mathbb{Z}$. Тогда существуют факторгруппы

$$
G_{l}=\bar{G} / \mathscr{Z}_{l}, \quad G_{p}=G_{l} / \mathscr{Z}_{p}, \quad G^{\text {ad }}=G_{l} / \mathscr{Z}\left(G_{l}\right),
$$

где $\mathscr{Z}\left(G_{l}\right)$ - центр $G_{l}$ и $\mathscr{Z}\left(G_{l}\right) \sim \mu_{p}=\mathscr{Z}(\bar{G}) / \mathscr{Z}_{l}$. 
ТАБлицА 2. Центры универсальных накрывающих групп $\left(\mu_{N}=\mathbb{Z} / N \mathbb{Z}\right)$

\begin{tabular}{|c|c|c|}
\hline $\bar{G}$ & $\operatorname{Lie}(\bar{G})$ & $\mathscr{Z}(\bar{G})$ \\
\hline $\operatorname{SL}(n, \mathbb{C})$ & $A_{n-1}$ & $\mu_{n}$ \\
$\operatorname{Spin}_{2 n+1}(\mathbb{C})$ & $B_{n}$ & $\mu_{2}$ \\
$\operatorname{Sp}_{n}(\mathbb{C})$ & $C_{n}$ & $\mu_{2}$ \\
$\operatorname{Spin}_{4 n}(\mathbb{C})$ & $D_{2 n}$ & $\mu_{2} \oplus \mu_{2}$ \\
$\operatorname{Spin}_{4 n+2}(\mathbb{C})$ & $D_{2 n+1}$ & $\mu_{4}$ \\
$E_{6}(\mathbb{C})$ & $E_{6}$ & $\mu_{3}$ \\
$E_{7}(\mathbb{C})$ & $E_{7}$ & $\mu_{2}$ \\
\hline
\end{tabular}

Группа $\bar{G}=\operatorname{Spin}_{4 n}(\mathbb{C})$ имеет нетривиальный центр

$$
\mathscr{Z}\left(\operatorname{Spin}_{4 n}\right)=\left(\mu_{2}^{\mathrm{L}} \times \mu_{2}^{\mathrm{R}}\right), \quad \mu_{2}=\mathbb{Z} / 2 \mathbb{Z},
$$

где три подгруппы описываются в терминах генераторов:

$$
\mu_{2}^{\mathrm{L}}=\{(1,1),(-1,1)\}, \quad \mu_{2}^{\mathrm{R}}=\{(1,1),(1,-1)\}, \quad \mu_{2}^{\mathrm{diag}}=\{(1,1),(-1,-1)\} .
$$

Следовательно, существуют три промежуточные подгруппы между группами $\bar{G}=\operatorname{Spin}_{4 n}(\mathbb{C})$ и $G^{\mathrm{ad}}$ :

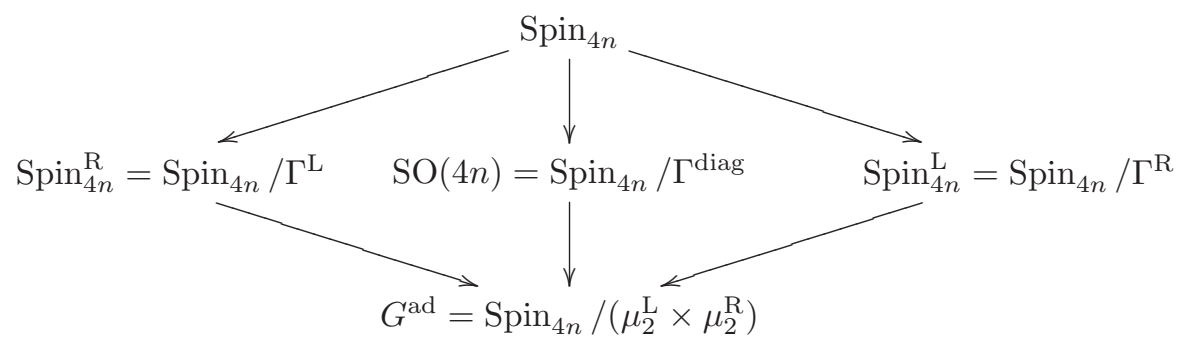

Характеры и кохарактеры. Пусть $\mathscr{H}$ - подгруппа Картана, $\mathscr{H} \subset G$. Определим группу характеров ${ }^{8}$

$$
\Gamma(G)=\left\{\chi: \mathscr{H} \rightarrow \mathbb{C}^{*}\right\} .
$$

Эта группа отождествляется с решеткой в $\mathfrak{h}^{*}$ следующим образом. Пусть $\mathbf{x}=$ $\left(x_{1}, x_{2}, \ldots, x_{n}\right)$ - элемент $\mathfrak{h}$ и $\exp (2 \pi i \mathbf{x}) \in \mathscr{H}$. Зададим $\gamma \in V^{*}$ таким, что $\chi_{\gamma}=\exp (2 \pi i\langle\gamma, \mathbf{x}\rangle) \in \Gamma(G)$. Тогда

$$
\Gamma(\bar{G})=P, \quad \Gamma\left(G^{\mathrm{ad}}\right)=Q
$$

и $\Gamma\left(G^{\mathrm{ad}}\right) \subseteq \Gamma\left(G_{l}\right) \subseteq \Gamma(\bar{G})$. Фундаментальные веса $\varpi_{k}(k=1, \ldots, n)$ и простые корни $\alpha_{k}$ образуют базисы в $\Gamma(\bar{G})$ и $\Gamma\left(G^{\mathrm{ad}}\right)$ соответственно. Пусть $\mathscr{Z}(\bar{G})$ - циклическая группа и $p$ является делителем $\operatorname{ord}(\mathscr{Z}(\bar{G}))$ таким, что $l=\operatorname{ord}(\mathscr{Z}(\bar{G})) / p$. Тогда решетка $\Gamma\left(G_{l}\right)$ есть

$$
\Gamma(G)=Q+\varpi \mathbb{Z}, \quad p \varpi \in Q .
$$

${ }^{8}$ Голоморфные отображения $\mathscr{H}$ в $\mathbb{C}^{*}$ такие, что $\chi(x y)=\chi(x) \chi(y)$ для $x, y \in \mathscr{H}$. 
Определим двойственные группы кохарактеров $t(G)=\Gamma^{*}(G)$ голоморфными отображениями:

$$
t(G)=\left\{\mathbb{C}^{*} \rightarrow \mathscr{H}\right\}
$$

или, что эквивалентно,

$$
t(G)=\left\{\mathbf{x} \in \mathfrak{h} \mid \chi\left(e^{2 \pi i \mathbf{x}}\right)=1\right\} .
$$

Общий элемент $t(G)$ имеет вид

$$
z^{\gamma}=\exp (2 \pi i \gamma \log z) \in \mathscr{H}_{G}, \quad \gamma \in \Gamma^{*}(G), \quad z \in \mathbb{C}^{*} .
$$

В частности, группы $t(\bar{G})$ и $t\left(G^{\text {ad }}\right)$ отождествляются с решетками кокорней и ковесов:

$$
t(\bar{G})=Q^{\vee}, \quad t\left(G^{\mathrm{ad}}\right)=P^{\vee},
$$

a $t(\bar{G}) \subseteq t\left(G_{l}\right) \subseteq t\left(G^{\mathrm{ad}}\right)$. Из (А.26) следует, что

$$
t(G)=Q^{\vee}+\varpi^{\vee} \mathbb{Z}, \quad l \varpi^{\vee} \in Q^{\vee} .
$$

Подрешетка $t\left(G_{l}\right) \subset P^{\vee}$ задает аффинную группу Вейля

$$
W_{t(G)}=t(G) \rtimes W
$$

(см. (А.12), (А.14)). Центр $\mathscr{Z}(G)$ группы $G$ изоморфен фактору:

$$
\mathscr{Z}(G) \sim P^{\vee} / t(G)
$$

а $\pi_{1}(G) \sim t(G) / Q^{\vee}$. В частности,

$$
\mathscr{Z}(\bar{G})=P^{\vee} / t(\bar{G}) \sim P^{\vee} / Q^{\vee}
$$

Аналогично, фундаментальная группа для $G^{\text {ad }}$ есть $\pi_{1}\left(G^{\mathrm{ad}}\right) \sim t\left(G^{\mathrm{ad}}\right) / Q^{\vee} \sim$ $P^{\vee} / Q^{\vee}$. Тройка данных $(R, t(G), \Gamma(G))$ называется данными корней.

Параболические подгруппы и многообразия флагов. Пусть П' - подмножество простых корней $\Pi^{\prime} \subset \Pi(\mathfrak{g})$ и $\mathfrak{g}^{\prime}-$ полупростая подалгебра в $\mathfrak{g}$, отвечающая П'. Пусть $\mathfrak{h}^{\prime}-$ подалгебра Картана в $\mathfrak{g}^{\prime}$. Тогда алгебра Картана $\mathfrak{h} \subset \mathfrak{g}$ имеет разложение $\mathfrak{h}=\mathfrak{h}^{\prime} \oplus \widetilde{\mathfrak{h}}$. Аналогично, для коалгебр мы имеем

$$
\mathfrak{h}^{*}=\mathfrak{h}^{*} \oplus \widetilde{\mathfrak{h}}^{*}
$$

Эти данные задают параболическую подалгебру $\mathfrak{p}$ в $\mathfrak{g}$. Пусть $R^{\prime}-$ корни, отвечающие $\Pi^{\prime}$, и $\widetilde{R}=R \backslash R^{\prime}$. Параболическая подалгебра есть полупрямая сумма редуктивной подалгебры (подалгебры Леви) и нильпотентного идеала:

$$
\mathfrak{p}=\mathfrak{s}\left(\Pi^{\prime}\right) \oplus \mathfrak{n}^{+}\left(\widetilde{R}^{+}\right), \quad \mathfrak{s}=\mathfrak{g}^{\prime}\left(\Pi^{\prime}\right) \oplus \widetilde{\mathfrak{h}}, \quad \mathfrak{n}^{+}=\bigoplus_{\alpha \in \widetilde{R}^{+}} c_{\alpha} E_{\alpha}
$$


Рассмотрим разложение $\mathfrak{g}$ на параболическую и нильпотентную подалгебры:

$$
\mathfrak{g}=\mathfrak{p} \oplus \mathfrak{n}^{-}, \quad \mathfrak{n}^{-}=\bigoplus_{\alpha \in \widetilde{R}^{-}} c_{\alpha} E_{\alpha} .
$$

Пусть $P \subset G$ - параболическая подгруппа, задаваемая $\mathfrak{p}, N^{+}$- унипотентная подгруппа $\mathfrak{n}^{+}=\operatorname{Lie}\left(N^{+}\right)$, а $L-$ подгруппа Леви с алгеброй Ли

$$
\operatorname{Lie}(L)=\mathfrak{s}=\mathfrak{g}^{\prime} \oplus \widetilde{\mathfrak{h}}, \quad(\text { A.36) }
$$

Параболическая подгруппа $P$ есть полупрямое произведение

$$
P=L \ltimes N^{+}, \quad \operatorname{Lie}\left(N^{+}\right)=\mathfrak{n}^{+} .
$$

Если $\Pi^{\prime}=\varnothing$, то $P=B$ есть подгруппа Бореля. Для $G$ имеем разложения Брюа

$$
G=\bigcup_{w \in W} N^{-} w P=\bigcup_{w \in W} N^{+} w P, \quad \operatorname{Lie}\left(N^{-}\right)=\mathfrak{n}^{-} .
$$

Фактор $G / P=$ Flag называется многообразием $G$-флагов. $N^{+}$-орбиты элемента $w$ в Flag суть клетки Шуберта. Если $P=B$ - подгруппа Бореля, то флаг Flag $=G / B$ является полным $G$-флагом. Пусть $G^{\text {сотр }}-$ компактная форма комплексной группы $G$. Имеется разложение Ивасавы

$$
G=G^{\mathrm{comp}} P, \quad G^{\mathrm{comp}} \cap P=L^{\mathrm{comp}}, \quad L^{\mathrm{comp}}=L \cap G^{\mathrm{comp}} .
$$

Тем самым, многообразия флагов - это орбиты компактных групп:

$$
\text { Flag }=G / P \sim G^{\mathrm{comp}} / L^{\mathrm{comp}} .
$$

Параболические подалгебры задаются с помощью фундаментальных ковесов. Пусть $\gamma \in \Upsilon^{\vee}$ (А.9). Ее алгебра Ли имеет вид (см. (А.36))

$$
\operatorname{Lie}\left(P_{\gamma}\right)=\mathfrak{p}_{\gamma}=\mathfrak{s}_{\gamma}\left(\Pi^{\prime}\right) \oplus \mathfrak{n}_{\gamma}^{+}\left(\widetilde{R}^{+}\right), \quad \Pi^{\prime}=\{\alpha \in \Pi \mid\langle\alpha, \gamma\rangle=0\} .
$$

Соответствующая параболическая подгруппа $P_{\gamma}$ максимальна, и полупростая подалгебра $\mathfrak{g}^{\prime}$ (А.38) имеет ранг $n-1$. Нам понадобится специальный класс параболических подалгебр. Будем называть параболические подалгебры и соответствующие алгебры Леви допустимыми, если

$$
\langle\gamma, \alpha\rangle=1 \quad \forall \alpha \in \widetilde{R}^{+} .
$$

Определим допустимые фундаментальные ковеса

$$
\widehat{\Upsilon}^{\vee}=\left\{\gamma \in \Upsilon^{\vee} \mid \gamma \notin \Pi^{\vee},\langle\gamma, \alpha\rangle=1 \forall \alpha \in \widetilde{R}^{+}\right\} .
$$

Заметим, что для классических алгебр каждый нетривиальный фундаментальный ковес $\gamma \in \Upsilon^{\vee}, \gamma \notin \Pi^{\vee}$, задает допустимую параболическую подалгебру и подалгебру Леви. Это не так для исключительных алгебр $e_{6}$ и $e_{7}$.

Используя обозначения для корней и весов из [83], приведем допустимые фундаментальные ковеса и полупростые компоненты допустимых алгебр Леви для простых групп Ли с нетривиальными центрами (табл. 3). 
ТАБлицА 3. Допустимые подалгебры Леви и многообразия флагов

\begin{tabular}{|c|c|c|c|}
\hline $\bar{G}$ & $\widehat{\Upsilon}^{\vee}$ & $\mathfrak{g}^{\prime}$ & $G / P$ \\
\hline $\mathrm{SL}(n, \mathbb{C})$ & $\Upsilon^{\vee}$ & $\mathrm{sl}(n-p) \oplus \operatorname{sl}(p)$ & $\mathrm{Gr}(n, p)=\mathrm{S}(\mathrm{U}(n-p) \times \mathrm{U}(p))$ \\
$\operatorname{Spin}_{2 n+1}(\mathbb{C})$ & $\varpi_{1}^{\vee}$ & $\mathrm{so}(2 n-1)$ & $\mathrm{SO}(2 n+1, \mathbb{R}) / \mathrm{SO}(2 n-1, \mathbb{R}) \times \mathrm{SO}(2)$ \\
$\operatorname{Sp}_{n}(\mathbb{C})$ & $\varpi_{1}^{\vee}$ & $\mathrm{sp}(n-1)$ & $\mathrm{Sp}(n, \mathbb{R}) / \mathrm{Sp}(n-1, \mathbb{R}) \times \mathrm{SO}(2)$ \\
$\operatorname{Spin}_{2 n}(\mathbb{C})$ & $\varpi_{1}^{\vee}$ & $\mathrm{so}(2 n-2)$ & $\mathrm{SO}(2 n, \mathbb{R}) / \mathrm{SO}(2 n-2, \mathbb{R}) \times \mathrm{SO}(2)$ \\
$\operatorname{Spin}_{2 n}(\mathbb{C})$ & $\varpi_{n-1, n}^{\vee}$ & $\mathrm{sl}(n)$ & $\mathrm{SO}(2 n, \mathbb{R}) / \mathrm{SU}(n-1) \times \mathrm{SO}(2)$ \\
$E_{6}(\mathbb{C})$ & $\varpi_{1}^{\vee}$ & $\mathrm{so}(10)$ & $E_{6}^{\text {comp }} / \mathrm{SO}(10) \times \mathrm{SO}(2)$ \\
$E_{7}(\mathbb{C})$ & $\varpi_{7}^{\vee}$ & $E_{6}$ & $E_{7}^{\text {comp }} / E_{6}^{\text {comp }} \times \mathrm{SO}(2)$ \\
\hline
\end{tabular}

Если ослабить условие (А.44) до

$$
\langle\gamma, \alpha\rangle=1 \quad \text { или } \quad 0 \quad \forall \alpha \in \widetilde{R}^{+},
$$

то любая параболическая подалгебра допустимой параболической алгебры допустима. В частности, подалгебры Бореля допустимы.

Коприсоединенные орбиты. Кокасательное расслоение $T^{*} G$ к группе $G$ снабжено симплектической формой

$$
\omega=\delta\left\langle a, g^{-1} \delta g\right\rangle, \quad a \in \mathfrak{g}^{*}, \quad g \in G .
$$

Эта форма инвариантна относительно действий параболической подгруппы $f_{\text {out }}, f_{\text {int }} \in P$ :

$$
\begin{aligned}
\operatorname{Ad}_{f_{\text {int }}^{*}}^{*}(a) & =f_{\text {int }}^{-1} a f_{\text {int }}, \quad g \rightarrow g f_{\text {int }}, \\
a & \rightarrow a, \quad g \rightarrow f_{\text {out }} g .
\end{aligned}
$$

Элементы $\varepsilon_{\text {int }}, \varepsilon_{\text {out }} \in \mathfrak{p}$ генерируют векторные поля по преобразованиям (А.48), (А.49). Их гамильтонианы $F_{\text {int }}, F_{\text {out }}\left(\delta F_{\text {int,out }}=i_{\varepsilon_{\text {int }, \text { out }}} \omega\right)$ имеют вид

$$
F_{\text {int }}=\left\langle\varepsilon_{\text {int }}, a\right\rangle, \quad F_{\text {out }}=\left\langle\varepsilon_{\text {out }}, g a g^{-1}\right\rangle .
$$

Моменты этих действий принимают значения в $\mathfrak{p}_{a}^{*}=\mathfrak{s} \oplus \mathfrak{n}_{a}^{-}$(см. (А.36)). Момент, отвечающий int-действию, равен $\mu_{\mathrm{int}}=\left.\operatorname{Pr}\right|_{\mathfrak{p}^{*}}(a)$. Зафиксируем его как $\mu_{\mathrm{int}}=\nu \in \mathfrak{s .}$ Это означает, что решение уравнения момента есть

$$
a=\nu+\xi, \quad \xi \in \mathfrak{n}^{+}
$$

где $\nu$ фиксировано, а $\xi$ - произвольный элемент $\mathfrak{n}^{+}$.

Коприсоединенное действие $P$ сохраняет $\nu \in \mathfrak{s}$ и редуцированное симплектическое многообразие

$$
T^{*} G / / P=\mu_{\text {int }}^{-1}(\nu) / P .
$$

Из (А.48) и (А.51) следует, что симплектический фактор $T^{*} G / / P$ задается парами $(g, \nu+\xi), g \in G, \xi \in \mathfrak{n}^{+}$, с отношением эквивалентности

$$
\left(g b, \operatorname{Ad}_{b}^{*}(\nu+\xi)\right) \sim(g, \nu+\xi), \quad b \in P .
$$


Заметим, что коприсоединенное действие $P$ на $\xi \in \mathfrak{n}^{+}$является из-за наличия $\nu$ аффинным. Группа $P$ действует свободно на $\mathfrak{n}^{+}$. Это означает, что $T^{*} G / / P-$ главное однородное пространство $P H / T^{*}(G / P)$ над кокасательным расслоением к многообразию флагов $G / P$. Само кокасательное расслоение появляется при $\nu=0$.

Зафиксируем калибровку $P$-действия, выбрав $\xi=0$. Как следует из (А.36) и (А.39), подгруппа Леви $L$ сохраняет $\xi=0$. Тогда из (А.53) находим, что

$$
\mathscr{O}_{\nu}=\left(\mathrm{Ad}^{*}\right)_{G}^{-1} \nu=G / L
$$

Форма $\omega$ на $T^{*} G(\mathrm{~A} .47)$ становится равной форме Кириллова-Костанта

$$
\omega^{\mathrm{KK}}=\left\langle\nu, g^{-1} \delta g g^{-1} \delta g\right\rangle
$$

Размерность орбиты равна

$$
\operatorname{dim}\left(\mathscr{O}_{\mathfrak{p}}\right)=\sharp(\widetilde{R})
$$

Аффинные алгебры Ли [71]. Аффинная система корней определяется так:

$$
\begin{aligned}
& R^{\mathrm{aff}}=\left\{\begin{array}{l|l}
\widehat{\alpha}=\alpha+n & \begin{array}{l}
\alpha \in R \cup\{0\}, n \in \mathbb{Z} \backslash\{0\}, \\
\alpha \in R, \\
n=0
\end{array}
\end{array}\right\}, \\
& R_{+}^{\mathrm{aff}}=R^{\mathrm{aff}} \text { для } n>0 \text { или для } \alpha \in R^{+}, n=0, \quad R_{-}^{\mathrm{aff}}=R^{\mathrm{aff}} \backslash R_{+}^{\mathrm{aff}} .
\end{aligned}
$$

Аффинная алгебра Ли представляется в виде $L(\mathfrak{g}) \oplus \mathbb{C c} \oplus \mathbb{C d}$, где

$$
L(\mathfrak{g})=\mathfrak{g} \otimes \mathbb{C}\left[t^{-1}, t\right]=\left\{\sum_{k} x_{k} t^{k}, x_{k} \in \mathfrak{g}\right\},
$$

c - генератор центра, a $\mathbf{d}$ - производная $L(\mathfrak{g})$. Аффинные корни отвечают аффинным корневым подпространствам в $L(\mathfrak{g})$ :

$$
E_{\widehat{\alpha}}=E_{\alpha} t^{n}, \quad H_{\widehat{\alpha}}=H_{\alpha} t^{n} \quad(n \neq 0) .
$$

Пусть $\mathscr{H}$ - подгруппа Картана в $G$. Реализуем группу Вейля $W$ для $\mathfrak{g}$ как фактор $\mathscr{N}(\mathscr{H}) / \mathscr{C}(\mathscr{H})$, где $\mathscr{N}(\mathscr{H})(\mathscr{C}(\mathscr{H}))-$ нормализатор (централизатор) $\mathscr{H}$. Определим два типа аффинных групп Вейля:

$$
\begin{aligned}
& W_{P}=\left\{\widehat{w}=w t^{\gamma}, w \in W, \gamma \in P^{\vee}\right\}, \\
& W_{Q}=\left\{\widehat{w}=w t^{\gamma}, w \in W, \gamma \in Q^{\vee}\right\} .
\end{aligned}
$$

Они действуют на корневые векторы как $E_{\widehat{\alpha}}=E_{\alpha} t^{n} \rightarrow E_{\widehat{w}(\widehat{\alpha})}=E_{w(\alpha)} t^{n+\langle\gamma, \alpha\rangle}$. 
Группы петель [70]. Пусть $L(G)$ - группа петель алгебры петель (А.58):

$$
\left.L(G)=G \otimes \mathbb{C}\left[t^{-1}, t\right]\right]=\left\{\sum_{k} g_{k} t^{k}, g_{k} \in G\right\} .
$$

Определим подгруппы петель

$$
\begin{aligned}
L^{+}(G) & \left.=\left\{g_{0}+g_{1} t+\cdots=g_{0}+t L[t]\right]\right\}, & & g_{j} \in G, \quad g_{0} \in P, \\
N^{-}(G) & =\left\{n_{-}+g_{1} t^{-1}+\cdots=n_{-}+t^{-1} L\left[t^{-1}\right]\right\}, & & n_{-} \in N^{-}, \\
N^{+}(G) & \left.=\left\{n_{+}+g_{1} t+\cdots=n_{+}+t L[t]\right]\right\}, & & n_{+} \in N^{+},
\end{aligned}
$$

где $\operatorname{Lie}\left(N^{+}\right)=\mathfrak{n}^{+}=\sum_{\alpha \in \widetilde{R}^{+}} c_{\alpha} E_{\alpha}($ А.36). Как следует из (А.63),

$$
L^{+}(G)=L \ltimes N^{+}(G),
$$

где $L$ - подгруппа Леви.

Существуют петлевые аналоги разложения Брюа (А.40). Разложение подалгебры Картана (А.35) задает подрешетку ковесов

$$
\widetilde{P}^{\vee}=\left\{\gamma \in P^{\vee} \mid\langle\gamma, \alpha\rangle=0, \alpha \in \mathfrak{h}^{\prime}\right\} .
$$

Подгруппа $\widetilde{W}_{P}$ аффинной группы Вейля $W_{P}$ генерируется группой Вейля $W^{\prime}=$ $W^{\prime}\left(\mathfrak{g}^{\prime}\right)$ с $\mathfrak{g}^{\prime}$ из $(\mathrm{A} .35)$ и подрешеткой $P^{\prime} \vee P^{\vee}$. Рассмотрим фактор $\widetilde{W}_{P}=$ $W_{P} / W_{P}^{\prime}$. Аналогично определим $\widetilde{W}_{Q}=W_{Q} / W_{Q}^{\prime}$ и $\widetilde{W}_{t(G)}=W_{t(G)} / W_{t(G)}^{\prime}$. По аналогии с (А.40) аффинное разложение Брюа принимает вид

$$
\begin{aligned}
L\left(G^{\mathrm{ad}}\right) & =\bigcup_{\widehat{w} \in \widetilde{W}_{P}} N^{-}\left(G^{\mathrm{ad}}\right) \widehat{w} L^{+}\left(G^{\mathrm{ad}}\right)=\bigcup_{\widehat{w} \in \widetilde{W}_{P}} N^{+}\left(G^{\mathrm{ad}}\right) \widehat{w} L^{+}\left(G^{\mathrm{ad}}\right), \\
L(\bar{G}) & =\bigcup_{\widehat{w} \in \widetilde{W}_{Q}} N^{-}(\bar{G}) \widehat{w} L^{+}(\bar{G})=\bigcup_{\widehat{w} \in \widetilde{W}_{Q}} N^{+}(\bar{G}) \widehat{w} L^{+}(\bar{G}), \\
L(G) & =\bigcup_{\widehat{w} \in \widetilde{W}_{t(G)}} N^{-}(G) \widehat{w} L^{+}(G)=\bigcup_{\widehat{w} \in \widetilde{W}_{t(G)}} N^{+}(G) \widehat{w} L^{+}(G) .
\end{aligned}
$$

Элемент $g(t) \in L(G)$ может иметь монодромию $g\left(t e^{2 \pi i}\right)=g(t) \zeta, \zeta=\mathbf{e}(\xi)$, где $\xi$ - представитель из фактора $P^{\vee} / t(G) \sim \mathscr{Z}(G)$ (А.33). Определим подмножество петель $L_{\zeta}(G)$, гомотопных $\mathbf{e}(\xi)$. Тогда получим разложение

$$
L(G)=\bigcup_{\zeta \in \mathscr{Z}(G)} L_{\zeta}(G) .
$$

Назовем афбинным многообразием флагов фактор

$$
\text { Flag }^{\text {aff }}=L(G) / L^{+}(G) .
$$

$N^{+}(G)$-орбита элемента $\widehat{w}$ в Flag $^{\text {aff }}$,

$$
C_{\widehat{w}}=\left\{n(t) \widehat{w} L^{+}(G) \mid n(t) \in N^{+}(G)\right\},
$$


называется аффинной клеткой Шуберта. Из (А.66)-(А.68) находим, что

$$
\text { Flag }^{\text {aff }}=\bigcup_{\widehat{w} \in W_{P, Q}} C_{\widehat{w}} .
$$

Размерность аффинной клетки Шуберта равна

$$
\operatorname{dim} C_{\widehat{w}}=l(\widehat{w})
$$

где $l(\widehat{w})$ есть длина $\widehat{w}$. Это число отрицательных аффинных корней (А.57), которые преобразуются в положительные под действием $\widehat{w}$.

\section{Приложение В. Обобщенный синус-базис в простых алгебрах Ли}

В этом приложении мы кратко опишем конструкцию, предложенную в [45].

Пусть $\mathfrak{g}$ - комплексная простая алгебра Ли, $\mathfrak{h}$ - подалгебра Картана, а $R$ система корней. Имеем разложение

$$
\mathfrak{g}=\mathfrak{h}+\mathfrak{l}, \quad \mathfrak{l}=\sum_{\beta \in R} \mathfrak{R}_{\beta}, \quad \operatorname{dim}_{\mathbb{C}} \mathfrak{R}_{\beta}=1 .
$$

Базис Шевалле в $\mathfrak{g}$ генерируется набором

$$
\left\{E_{\beta_{j}} \in \mathfrak{R}_{\beta_{j}}, \beta_{j} \in R ; H_{\alpha_{k}} \in \mathfrak{h}, \alpha_{k} \in \Pi\right\} \text {, }
$$

где $H_{\alpha_{k}}$ определяются из коммутационных соотношений

$$
\begin{aligned}
& {\left[E_{\alpha_{k}}, E_{-\alpha_{k}}\right]=H_{\alpha_{k}}, \quad\left[H_{\alpha_{k}}, E_{ \pm \alpha_{j}}\right]=a_{k j} E_{ \pm \alpha_{k}}, \quad \alpha_{k}, \alpha_{j} \in \Pi,} \\
& {\left[H_{\alpha_{j}}, E_{\alpha_{k}}\right]=a_{k j} E_{\alpha_{k}}, \quad\left[E_{\alpha}, E_{\beta}\right]=C_{\alpha, \beta} E_{\alpha+\beta} .}
\end{aligned}
$$

Перейдем от базиса Шевалле (В.2) к новому базису, удобному для описания расслоений с нетривиальными характеристическими классами. Будем называть его обобщенным синус-базисом (GS-базисом), так как для случая $A_{n}$ и расслоения степени один он совпадает с базисом синус-алгебры (см., например, [120]).

Выберем элемент $\zeta \in \mathscr{Z}(\bar{G})$ порядка $l$ и соответствующий $\Lambda^{0} \in W$ из (5.4). Тогда, как и в п. 5.2, $\Lambda^{0}$ генерирует циклическую группу $\mu_{l}=\left(\Lambda^{0},\left(\Lambda^{0}\right)^{2}, \ldots\right.$, $\left.\left(\Lambda^{0}\right)^{l}=1\right)$, изоморфную подгруппе $\mathscr{Z}(\bar{G})$. Так как $\Lambda^{0} \in W$, то этот элемент сохраняет систему корней $R$. Определим фактормножество $\mathscr{T}_{l}=R / \mu_{l}$. Тогда $R$ представляется в виде объединения $\mu_{l}$-орбит: $R=\bigcup_{\mathscr{T}_{l}} \mathscr{O}$. Обозначим через $\mathscr{O}(\bar{\beta})$ орбиту, начинающуюся с корня $\beta$ :

$$
\mathscr{O}(\bar{\beta})=\left\{\beta, \lambda(\beta), \ldots, \lambda^{l-1}(\beta)\right\}, \quad \bar{\beta} \in \mathscr{T}_{l} .
$$

Количество элементов орбиты $\mathscr{O}$ (длина $\mathscr{O}$ ) равно $l / p_{\alpha}=l_{\alpha}$, где $p_{\alpha}$ - делитель $l$. Пусть $\nu_{\alpha}$ - число орбит $\mathscr{O}_{\bar{\alpha}}$ длины $l_{\alpha}$. Тогда $\sharp(R)=\sum \nu_{\alpha} l_{\alpha}$. Заметим, что если $\mathscr{O}(\bar{\beta})$ имеет длину $l_{\beta}\left(l_{\beta} \neq 1\right)$, то элементы $\lambda^{k} \beta$ и $\lambda^{k+l_{\beta}} \beta$ совпадают. 
Базис в $\mathfrak{l}$ (В.1). Преобразуем корневой базис $\mathscr{E}=\left\{E_{\beta}, \beta \in R\right\}$ в l. Определим орбиту в $\mathscr{E}$

$$
\mathscr{E}_{\bar{\beta}}=\left\{E_{\beta}, E_{\lambda(\beta)}, \ldots, E_{\lambda^{l-1}(\beta)}\right\}
$$

отвечающую $\mathscr{O}(\bar{\beta})$. Снова $\mathscr{E}=\bigcup_{\bar{\beta} \in \mathscr{T}_{l}} \mathscr{E} \bar{\beta}$.

Для $\mathscr{O}(\bar{\beta})$ зададим множество целых чисел

$$
J_{p_{\alpha}}=\left\{a=m p_{\alpha} \mid m \in \mathbb{Z}, a \text { определено по модулю } l\right\} \quad\left(p_{\alpha}=\frac{l}{l_{\alpha}}\right) .
$$

“Преобразование Фурье" корневого базиса на орбите $\mathscr{O}(\bar{\beta})$ определим формулой

$$
\mathfrak{t} \frac{k}{\beta}=\frac{1}{\sqrt{l}} \sum_{m=0}^{l-1} \omega^{m a} E_{\lambda^{m}(\beta)}, \quad \omega=\exp \left(\frac{2 \pi i}{l}\right), \quad a \in J_{\beta} .
$$

Это преобразование обратимо:

$$
E_{\lambda^{k}(\beta)}=\frac{1}{\sqrt{l}} \sum_{a \in J_{l}} \omega^{-k a} \mathfrak{t} \frac{k}{\beta}
$$

и, следовательно, имеем взаимно однозначное отображение $\mathscr{E}_{\beta} \leftrightarrow\left\{\mathfrak{t} \frac{k}{\beta}, a \in J_{\beta}\right\}$. Таким образом определяется новый базис

$$
\left\{\mathfrak{t}_{\bar{\beta}}\left(a \in J_{l}, \bar{\beta} \in \mathscr{T}_{l}\right)\right\} .
$$

Так как $\lambda\left(E_{\alpha}\right)=E_{\lambda(\alpha)}$, для $\Lambda \mathbf{e}(\mathbf{u})$ имеем $\left(\mathbf{u} \in \widetilde{\mathfrak{h}}_{0}\right)$

$$
\operatorname{Ad}_{\Lambda}\left(\mathfrak{t}_{\frac{k}{\beta}}\right)=\mathbf{e}\left(\langle\mathbf{u}, \beta\rangle-\frac{a}{l}\right) \mathfrak{t} \frac{k}{\beta}, \quad \mathbf{e}(x)=\exp (2 \pi i x) .
$$

Это означает, что $\mathfrak{t}_{\bar{\beta}}\left(\bar{\beta} \in \mathscr{T}_{l}\right)$ - часть базиса в $\mathfrak{g}_{l-a}(5.11)$. Более того,

$$
\operatorname{Ad}_{\mathscr{Q}}\left(\mathfrak{t} \frac{k}{\beta}\right)=\mathbf{e}(\langle\kappa, \beta\rangle) \mathfrak{t} \frac{k}{\beta} .
$$

Эти соотношения следуют из (5.3). Учтем также, что $\mathscr{Q}$ и $\Lambda$ коммутируют в присоединенном представлении и $\mathbf{e}(x) E_{\alpha} \mathbf{e}(-x)=\mathbf{e}(\langle x, \alpha\rangle) E_{\alpha}$ для $x \in \widetilde{\mathfrak{h}}_{0}$.

Выбирая другой элемент $\Lambda^{\prime}$, генерирующий подгруппу $\mathscr{Z}_{l_{1}^{\prime}}\left(l^{\prime} \neq l\right)$, получим другой набор орбит и другой базис. Количество различных базисов равно количеству неизоморфных подгрупп в $\mathscr{Z}(\bar{G})$.

Форма Киллинга. Рассмотрим две орбиты $\mathscr{O}(\bar{\alpha})$ и $\mathscr{O}(\bar{\beta})$, проходящие через $E_{\alpha}$ и $E_{\beta}$. Предположим, что существует целое число $r$ такое, что $\alpha=-\lambda^{r}(\beta)$. Это подразумевает, что элементы двух орбит связаны: $\lambda^{n}(\alpha)=-\lambda^{m}(\beta)$, если $m-n=r$. Иными словами, $-\beta \in \mathscr{O}(\bar{\alpha})$. В частности, это означает, что орбиты имеют одинаковые длины. Из (В.5) и (А.20) следует, что

$$
\left(\mathfrak{t}_{\bar{\alpha}}^{c_{1}}, \mathfrak{t}_{\bar{\beta}}^{c_{2}}\right)=\delta_{\alpha,-\lambda^{r}(\beta)} \delta^{\left(c_{1}+c_{2}, 0(\bmod l)\right)} \omega^{-r c_{1}} \frac{2 p_{\alpha}}{(\alpha, \alpha)},
$$


где $p_{\alpha}=l / l_{\alpha}$ и $l_{\alpha}$ - длина $\mathscr{O}(\bar{\alpha})$. В частности, $\left(\mathfrak{t}_{\bar{\alpha}}^{a}, \mathfrak{t}_{-\bar{\alpha}}^{-a}\right)=2 p_{\alpha} /(\alpha, \alpha)$. Далее нам понадобится дуальный базис $\mathfrak{T}_{\bar{\alpha}}$ :

$$
\left(\mathfrak{T}_{\bar{\alpha}_{1}}^{b_{1}}, \mathfrak{t}_{\bar{\alpha}_{2}}^{b_{2}}\right)=\delta^{\left(b_{1}+b_{2}, 0(\bmod l)\right)} \delta_{\bar{\alpha}_{1},-\bar{\alpha}_{2}}, \quad \mathfrak{T}_{\bar{\alpha}}^{b}=\mathfrak{t}_{-\bar{\alpha}}^{-b} \frac{(\alpha, \alpha)}{2 p_{\alpha}} .
$$

Форма Киллинга в этом базисе обратна (В.9):

$$
\left(\mathfrak{T}_{\bar{\alpha}_{1}}^{a_{1}}, \mathfrak{T}_{\bar{\alpha}_{2}}^{a_{2}}\right)=\delta_{\alpha_{1},-\lambda^{r}\left(\alpha_{2}\right)} \delta^{\left(a_{1}+a_{2}, 0(\bmod l)\right)} \omega^{r a_{1}} \frac{\left(\alpha_{1}, \alpha_{1}\right)}{2 p_{\alpha_{1}}} .
$$

В частности,

$$
\left(\mathfrak{T}_{\alpha}^{a}, \mathfrak{T}_{-\alpha}^{-a}\right)=\frac{(\alpha, \alpha)}{2 p_{\alpha}} .
$$

Базис в подалгебре Картана. Почти такая же конструкция существует и в $\mathfrak{h}$. Пусть снова $\Lambda^{0}$ генерирует $\mu_{l}$. Так как $\Lambda^{0}$ сохраняет расширенный граф Дынкина, то его действие сохраняет и расширенную систему кокорней $\Pi^{\vee}$ ext $=$

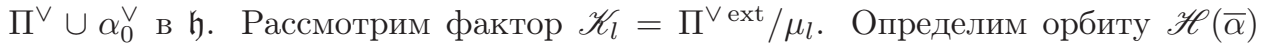

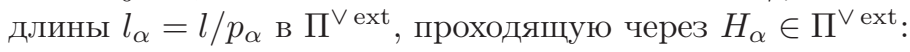

$$
\mathscr{H}(\bar{\alpha})=\left\{H_{\alpha}, H_{\lambda(\alpha)}, \ldots, H_{\lambda^{l-1}(\alpha)}\right\}, \quad \bar{\alpha} \in \mathscr{K}_{l}=\Pi^{\vee \operatorname{ext}} / \mu_{l} .
$$

Множество $\Pi^{\vee \text { ext } е с т ь ~ о б ъ е д и н е н и е ~} \mathscr{H}(\bar{\alpha})$ :

$$
\Pi^{\vee \operatorname{ext}}=\bigcup_{\bar{\alpha} \in \mathscr{K}_{l}} \mathscr{H}(\bar{\alpha})
$$

Определим "преобразование Фурье"

$$
\mathfrak{h}_{\bar{\alpha}}^{k}=\frac{1}{\sqrt{l}} \sum_{m=0}^{l-1} \omega^{m k} H_{\lambda^{m}(\alpha)}, \quad \omega=\exp \left(\frac{2 \pi i}{l}\right), \quad k \in J_{\alpha} \quad \text { (B.4). }
$$

Базис $\mathfrak{h} \frac{c}{\alpha}\left(c \in J_{\alpha}, \bar{\alpha} \in \mathscr{K}_{l}\right)$ переопределен в $\mathfrak{h}$. А именно, пусть $\mathscr{H}\left(\bar{\alpha}_{0}\right)$ - орбита, проходящая через минимальный кокорень $\left\{H_{\alpha_{0}}, H_{\lambda\left(\alpha_{0}\right)}, \ldots, H_{\lambda^{l-1}\left(\alpha_{0}\right)}\right\}$. Тогда элемент $\mathfrak{h}_{\bar{\alpha}_{0}}^{0}$ есть линейная комбинация элементов $\mathfrak{h}_{-\bar{\alpha}}^{0}(\alpha \in \Pi)$ и его следует исключить из базиса. Заменим базис $\Pi^{\vee}$ в $\mathfrak{h}$ на

$$
\mathfrak{h}_{\bar{\alpha}}^{c} \quad\left(c \in J_{\alpha}\right), \quad \begin{cases}\bar{\alpha} \in \widetilde{\mathscr{K}_{l}}=\mathscr{K}_{l} \backslash \mathscr{H}\left(\bar{\alpha}_{0}\right), & c=0, \\ \bar{\alpha} \in \mathscr{K}_{l}, & c \neq 0 .\end{cases}
$$

Как и ранее, существует взаимно однозначное соответствие $\Pi^{\vee} \leftrightarrow\left\{\mathfrak{h} \frac{c}{\alpha}\right\}$. Элементы $\left(\mathfrak{h} \frac{a}{\alpha}, \mathfrak{t}_{\alpha}^{k}\right)$ образуют GS-базис в $\mathfrak{g}_{l-a}(5.11)$.

Форма Киллинга. Форма Киллинга в базисе (В.13) находится из (А.19):

$$
\left(\mathfrak{h} \frac{k}{\alpha}, \mathfrak{h} \frac{b}{\beta}\right)=\delta^{(k+j, 0(\bmod l))} \mathscr{A}_{\alpha, \beta}^{k}, \quad \mathscr{A}_{\alpha, \beta}^{k}=\frac{2}{(\beta, \beta)} \sum_{s=0}^{l-1} \omega^{-s k} a_{\beta, \lambda^{s}(\alpha)},
$$


где $a_{\alpha, \beta}$ - матрица Картана (А.4). Двойственный базис генерируется элементами $\mathfrak{h} \frac{a}{\alpha}$ :

$$
\begin{gathered}
\left(\mathfrak{H}_{\bar{\alpha}}^{k}, \mathfrak{h}_{\bar{\beta}}^{j}\right)=\delta^{(k+j, 0(\bmod l))} \delta_{\alpha \beta}, \\
\mathfrak{H}_{\bar{\alpha}}^{k}=\sum_{\beta \in \Pi}\left(\mathscr{A}_{\alpha, \beta}^{k}\right)^{-1} \mathfrak{h}_{\bar{\beta}}^{-k}, \quad \mathfrak{h} \frac{k}{\beta}=\sum_{\alpha \in \Pi} \mathscr{A}_{\alpha, \beta}^{-k} \mathfrak{H}_{\bar{\alpha}}^{-k} .
\end{gathered}
$$

Форма Киллинга в нем имеет вид

$$
\left(\mathfrak{h}_{\bar{\alpha}_{1}}^{k_{1}}, \mathfrak{h}_{\bar{\alpha}_{2}}^{k_{2}}\right)=\delta^{\left(k_{1}+k_{2}, 0(\bmod l)\right)}\left(\mathscr{A}_{\bar{\alpha}_{1}, \bar{\alpha}_{2}}^{k_{1}}\right)^{-1} .
$$

В итоге мы определили GS-базис в $\mathfrak{g}$

$$
\left\{\mathfrak{t}_{\bar{\beta}}, \mathfrak{h}_{\bar{\alpha}}^{j} ;(k, \bar{\beta}, j, \bar{\alpha}) \text { определены в (В.6), (В.13) }\right\},
$$

и двойственный базис

$$
\left\{\mathfrak{T}_{\bar{\beta}}^{k}, \mathfrak{H}_{\bar{\alpha}}^{j} ;(k, \bar{\beta}, j, \bar{\alpha}) \text { определены в }(\mathrm{B} .10),(\mathrm{B} .15)\right\}
$$

вместе с формами Киллинга.

Коммутационные соотношения. Коммутационные соотношения в GS-базисе могут быть найдены из коммутационных соотношений в базисе Шевалле (А.17). Учитывая инвариантность структурных констант относительно действия группы Вейля, $C_{\lambda \alpha, \lambda \beta}=C_{\alpha, \beta}$, коммутационные соотношения в GS-базисе несложно получить из определения в базисе (B.5), (В.12). Для коммутатора корневых частей получим

$$
\left[\mathfrak{t}_{\alpha}^{a}, \mathfrak{t}_{\beta}^{b}\right]= \begin{cases}\frac{1}{\sqrt{l}} \sum_{s=0}^{l-1} \omega^{b s} C_{\alpha, \lambda^{s} \beta} \mathfrak{t}_{\alpha+\lambda^{s} \beta}^{a+b}, & \alpha \neq-\lambda^{s} \beta, \\ \frac{p_{\alpha}}{\sqrt{l}} \omega^{s b} \mathfrak{h}_{\alpha}^{a+b}, & \alpha=-\lambda^{s} \beta .\end{cases}
$$

Коммутаторы корневых и картановских генераторов:

$$
\begin{aligned}
{\left[\mathfrak{h}_{\alpha}^{k}, \mathfrak{t}_{\beta}^{m}\right] } & =\frac{1}{\sqrt{l}} \sum_{s=0}^{l-1} \omega^{-k s} \frac{2\left(\alpha, \lambda^{s} \beta\right)}{(\alpha, \alpha)} \mathfrak{t}_{\beta}^{k+m}, \\
{\left[\mathfrak{H}_{\alpha}^{k}, \mathfrak{t}_{\beta}^{m}\right] } & =\frac{1}{\sqrt{l}} \sum_{s=0}^{l-1} \omega^{-k s} \frac{(\alpha, \alpha)}{2}\left(\widehat{\alpha}, \lambda^{s} \beta\right) \mathfrak{t}_{\beta}^{k+m} .
\end{aligned}
$$

Здесь через $\widehat{\alpha}$ обозначены элементы, дуальные простым корням в подалгебре Картана:

$$
\left(\widehat{\alpha}_{i}, \beta_{j}\right)=\delta_{i j} .
$$

Более удобно использовать следующий нормированный базис в подалгебре Картана:

$$
\overline{\mathfrak{h}}_{\alpha}^{k}=\frac{(\alpha, \alpha)}{2} \mathfrak{h}_{\alpha}^{k}, \quad \overline{\mathfrak{H}}_{\alpha}^{k}=\frac{2}{(\alpha, \alpha)} \mathfrak{h}_{\alpha}^{k} .
$$


Такая репараметризация приводит к соотношениям

$$
\begin{aligned}
{\left[\overline{\mathfrak{h}}_{\alpha}^{k}, \mathfrak{t}_{\beta}^{m}\right] } & =\frac{1}{\sqrt{l}} \sum_{s=0}^{l-1} \omega^{-k s}\left(\alpha, \lambda^{s} \beta\right) \mathfrak{t}_{\beta}^{k+m}, \\
{\left[\overline{\mathfrak{H}}_{\alpha}^{k}, \mathfrak{t}_{\beta}^{m}\right] } & =\frac{1}{\sqrt{l}} \sum_{s=0}^{l-1} \omega^{-k s}\left(\widehat{\alpha}, \lambda^{s} \beta\right) \mathfrak{t}_{\beta}^{k+m} .
\end{aligned}
$$

Разложение картановских элементов в базисе простых корней дается следующей простой формулой:

$$
\overline{\mathfrak{h}}_{\beta}^{k}=\sum_{\alpha \in \Pi}(\widehat{\alpha}, \beta) \overline{\mathfrak{h}}_{\alpha}^{k}, \quad \beta \in R .
$$

Связь дуальных базисов следует из соотношения

$$
\sum_{\beta \in \Pi}(\widehat{\alpha}, \beta) \overline{\mathfrak{h}}_{\beta}^{k}=\sum_{\beta \in \Pi}(\alpha, \beta) \overline{\mathfrak{H}}_{\beta}^{k} .
$$

Картановские элементы обладают свойством симметрии:

$$
\overline{\mathfrak{h}}_{-\alpha}^{k}=-\overline{\mathfrak{h}}_{\alpha}^{k}, \quad \overline{\mathfrak{H}}_{-\alpha}^{k}=-\overline{\mathfrak{H}}_{\alpha}^{k} .
$$

Инвариантная подалгебра. Рассмотрим инвариантную подалгебру $\mathfrak{g}_{0}$. Она генерируется базисом $\left(\mathfrak{t}_{\bar{\beta}}^{0}, \mathfrak{h}_{\bar{\alpha}}^{0}\right)\left(\right.$ В.17). В частности, $\left\{\mathfrak{h}_{\bar{\alpha}}^{0}\right\}$ (В.12), (В.13) образует базис в картановской подалгебре $\widetilde{\mathfrak{h}}_{0} \subset \mathfrak{h}\left(\operatorname{dim} \widetilde{\mathfrak{h}}_{0}=p<n\right)$.

Перейдем от $\left\{\mathfrak{h}_{\bar{\alpha}}^{0}\right\}$ к специальному базису

$$
\widetilde{\Pi}^{\vee}=\left\{\widetilde{\alpha}_{k}^{\vee} \mid k=1, \ldots, p\right\}
$$

в $\widetilde{\mathfrak{h}}_{0}$. Он строится следующим образом. Рассмотрим подсистему простых кокорней

$$
\Pi_{1}^{\vee}=\Pi^{\operatorname{ext} \vee} \backslash \mathscr{O}\left(\bar{\alpha}_{0}^{\vee}\right)
$$

(см. (В.13)). Иными словами, $\Pi_{1}^{\vee}$ - подмножество простых кокорней, не содержащее простых кокорней из орбиты, проходящей через $\alpha_{0}$. Для $A_{N-1}, B_{n}, E_{6}$ и $E_{7}$ базис кокорней $\widetilde{\Pi}^{\vee}\left(\right.$ В.27) есть результат усреднения по $\lambda$-орбитам в $\Pi_{1}^{\vee}$ :

$$
\widetilde{\alpha}^{\vee}=\sum_{m=1}^{l-1} H_{\lambda^{m}(\alpha)}, \quad H_{\alpha} \in \Pi_{1}^{\vee} .
$$

В случаях $C_{n}$ и $D_{n}$ эта конструкция верна почти для всех кокорней, кроме последнего в диаграмме Дынкина (см. замечание 10.1 в [46]).

Рассмотрим дуальные векторы $\widetilde{\Pi}=\left\{\widetilde{\alpha}_{k} \mid k=1, \ldots, p,\left\langle\widetilde{\alpha}_{k}, \widetilde{\alpha}_{k}^{\vee}\right\rangle=2\right\}$ в $\widetilde{\mathfrak{h}}_{0}^{*}$.

ПРЕДЛОЖЕНИЕ В.1. Множество

$$
\widetilde{\Pi}=\left\{\widetilde{\alpha}_{k} \mid k=1, \ldots, p\right\}
$$

векторов из $\widetilde{\mathfrak{h}}_{0}^{*}$ является системой простых корней простой подалгебры Ли $\widetilde{\mathfrak{g}}_{0} \subset \mathfrak{g}_{0}$, заданной системой корней $\widetilde{R}=\widetilde{R}(\widetilde{\Pi})$ и матрицей Картана $\left\langle\widetilde{\alpha}_{k}, \widetilde{\alpha}_{j}^{\vee}\right\rangle$. 
Проверка этого утверждения выполнена в [46] перебором всех случаев.

Пусть $R_{1}=R_{1}\left(\Pi_{1}\right)$ - подмножество корней, генерируемых простыми корнями $\Pi_{1}=\Pi^{\text {ext }} \backslash \mathscr{O}\left(\alpha_{0}\right)$. Оно инвариантно относительно $\lambda$-действия. Система корней $\widetilde{R}$ в $\widetilde{\mathfrak{g}}_{0}$ отвечает $\lambda$-инвариантному множеству $R_{1}$. Рассмотрим дополнительное к нему множество корней $R \backslash R_{1}$ и множество орбит

$$
\mathscr{T}_{l}^{\prime}=\left(R \backslash R_{1}\right) / \mu_{l}
$$

Это подмножество среди всех орбит $\mathscr{T}_{l}=R / \mu_{l}$. Следовательно, $\mathscr{T}_{l}=\widetilde{R} \cup \mathscr{T}_{l}^{\prime}$. Заметим, что $\lambda$-инвариантная подалгебра $\mathfrak{g}_{0}$ содержит подпространство

$$
V=\left\{\sum_{\bar{\beta} \in \mathscr{T}_{l}^{\prime}} a_{\bar{\beta}} \mathrm{t}_{\bar{\beta}}^{0}, a_{\bar{\beta}} \in \mathbb{C}\right\} .
$$

Тогда $\mathfrak{g}_{0}$ есть сумма $\widetilde{\mathfrak{g}}_{0}$ и $V$ :

$$
\mathfrak{g}_{0}=\mathfrak{g}_{0}^{\prime} \oplus V
$$

Компоненты этого разложения ортогональны относительно формы Киллинга (В.14), а $V$ - представление $\mathfrak{g}_{0}^{\prime}$. В табл. 4 мы явно перечисляем $\mathfrak{g}_{0}$ для всех простых алгебр из нашего списка.

Пусть $\mathfrak{h}^{\prime}-$ подалгебра $\mathfrak{h}$ с базисом $\mathfrak{h}_{\bar{\alpha}}^{j}, j \neq 0$ (В.12), и $\widetilde{\mathfrak{h}}_{0}-$ подалгебра Картана в $\widetilde{\mathfrak{g}}_{0}$. Тогда

$$
\mathfrak{h}=\widetilde{\mathfrak{h}}_{0} \oplus \mathfrak{h}^{\prime}
$$

Информация об инвариантных подалгебрах собрана в табл. 4.

\begin{tabular}{|c|c|c|c|c|c|c|}
\hline$\Pi$ & $\mathscr{Z}(\bar{G})$ & $\varpi_{j}^{\vee}$ & $\Pi_{1}$ & $l=\operatorname{ord}(\Lambda)$ & $\widetilde{\mathfrak{g}}_{0}$ & $\mathfrak{g}_{0}$ \\
\hline 1 & 2 & 3 & 4 & 5 & 6 & 7 \\
\hline$A_{N-1}(N=p l)$ & $\mu_{N}$ & $\varpi_{N-1}^{\vee}$ & $\bigcup_{p=1}^{l} A_{p-1}$ & $N / p$ & $\mathrm{sl}_{p}$ & $\operatorname{sl}_{p} \bigoplus_{j=1}^{l-1} \mathrm{gl}_{p}$ \\
\hline$B_{n}$ & $\mu_{2}$ & $\varpi_{n}^{\vee}$ & $\mathrm{SO}_{2 n-1}$ & 2 & $\operatorname{so}(2 n-1)$ & so $(2 n)$ \\
\hline$C_{2 l}(l>1)$ & $\mu_{2}$ & $\varpi_{2 l}^{\vee}$ & $A_{2 l-1}$ & 2 & so $(2 l)$ & $\mathrm{gl}_{2 l}$ \\
\hline$C_{2 l+1}$ & $\mu_{2}$ & $\varpi_{2 l+1}^{\vee}$ & $A_{2 l}$ & 2 & $\mathrm{so}(2 l+1)$ & $\mathrm{gl}_{2 l+1}$ \\
\hline$D_{2 l+1}(l>1)$ & $\mu_{4}$ & $\varpi_{2 l+1}^{\vee}$ & $A_{2 l-2}$ & 4 & $\operatorname{so}(2 l-1)$ & $\mathrm{so}(2 l) \oplus \mathrm{so}(2 l) \oplus \underline{1}$ \\
\hline$D_{2 l+1}(l>1)$ & $\mu_{4}$ & $\varpi_{1}^{\vee}$ & $D_{2 l}$ & 2 & $\mathrm{so}(4 l-1)$ & $\mathrm{so}(4 l) \oplus \underline{1}$ \\
\hline$D_{2 l}(l>2)$ & $\mu_{2} \oplus \mu_{2}$ & $\varpi_{2 l}^{\vee}$ & $A_{2 l-1}$ & 2 & so $(2 l)$ & $\mathrm{so}(2 l) \oplus \operatorname{so}(2 l)$ \\
\hline$D_{2 l}(l>2)$ & $\mu_{2} \oplus \mu_{2}$ & $\varpi_{1}^{\vee}$ & $D_{2 l-1}$ & 2 & $\mathrm{so}(4 l-3)$ & $\mathrm{so}(4 l-2) \oplus \underline{1}$ \\
\hline$E_{6}$ & $\mu_{3}$ & $\varpi_{1}^{\vee}$ & $D_{4}$ & 3 & $\mathrm{~g}_{2}$ & $\mathrm{so}(8) \oplus 2 \cdot \underline{1}$ \\
\hline$E_{7}$ & $\mu_{2}$ & $\varpi_{7}^{\vee}$ & $\mathrm{e}_{6}$ & 2 & $\mathrm{f}_{4}$ & $\mathrm{e}_{6} \oplus \underline{1}$ \\
\hline
\end{tabular}

ТАБлицА 4. Инвариантные подалгебры $\widetilde{\mathfrak{g}}_{0}=\mathfrak{g}_{\widetilde{\Pi}}$ и $\mathfrak{g}_{0}$ простых алгебр Ли. Ковеса, генерирующие центральные элементы, содержатся в столбце 3 . 
В инвариантной простой алгебре $\mathfrak{g}_{0}^{\prime}$ вместо базиса $\left(\mathfrak{h} \frac{0}{\alpha}, \mathfrak{t}_{\frac{0}{\beta}}\right)$ можно рассмотреть базис Шевалле и включить его в GS-базис:

$$
\left\{\mathfrak{h}_{\bar{\alpha}}^{0}, \mathfrak{t}_{\bar{\beta}}^{0}\right\} \rightarrow\left\{\widetilde{\mathfrak{g}}_{0}=\left(H_{\widetilde{\alpha}}, \widetilde{\alpha} \in \widetilde{\Pi}, E_{\widetilde{\beta}}, \widetilde{\beta} \in \widetilde{R}\right), V=\left(\mathfrak{t}_{\bar{\beta}}^{0}, \bar{\beta} \in \mathscr{T}^{\prime}\right)\right\} .
$$

ЗАмечание В.1. Для любого $\xi \in Q^{\vee}$ решение уравнения (5.7) есть $\Lambda=\mathrm{Id}$. В этом случае $\mathfrak{g}_{0}^{\prime}=\mathfrak{g}$ и GS-базис совпадает с базисом Шевалле.

GS-базис как следствие канонического в $\mathfrak{h}$. Пусть $\left(e_{1}, e_{2}, \ldots, e_{n}\right)$ - канонический базис в $\mathfrak{h}\left(\left(e_{j}, e_{k}\right)=\delta_{j k}\right){ }^{9}$ Так как $\Lambda$ сохраняет $\mathfrak{h}$, можно рассмотреть действие $\mu_{l}$ на каноническом базисе. Определим орбиту длины $l_{s}=l / p_{s}$, проходящую через $e_{s}: \mathscr{O}(s)=\left\{e_{s}, \lambda\left(e_{s}\right), \ldots, \lambda^{(l-1)}\left(e_{s}\right)\right\}$.

Преобразование Фурье по $\mathscr{O}(s)$ имеет вид

$$
\mathfrak{h}_{s}^{c}=\frac{1}{\sqrt{l}} \sum_{m=0}^{l-1} \omega^{m c} \lambda^{m}\left(e_{s}\right), \quad c \in J_{p_{s}}, \quad \omega=\exp \left(\frac{2 \pi i}{l}\right),
$$

где $J_{p_{s}}=\left\{c=m p_{s}(\bmod l) \mid m \in \mathbb{Z}\right\}$. Рассмотрим фактор $\mathscr{C}_{l}=\left(e_{1}, e_{2}, \ldots, e_{n}\right) / \mu_{l}$. Тогда можно перейти от канонического базиса к GS-базису:

$$
\left(e_{1}, e_{2}, \ldots, e_{n}\right) \longleftrightarrow\left\{\mathfrak{h}_{s}^{c}, s \in \mathscr{C}_{l}\right\} .
$$

Форма Киллинга получается из (В.36):

$$
\left(\mathfrak{h}_{s_{1}}^{j_{1}}, \mathfrak{h}_{s_{2}}^{j_{2}}\right)=\delta_{\left(s_{1}, s_{2}\right)} \delta^{\left(j_{1},-j_{2}\right)} .
$$

Тогда дуальные генераторы равны

$$
\mathfrak{H}_{s}^{k}=\mathfrak{h}_{s}^{-k} .
$$

Коммутационные соотношения в $\mathfrak{g}$ :

$$
\begin{aligned}
{\left[\mathfrak{h}_{s}^{k_{1}}, \mathfrak{t}_{\frac{k_{2}}{\beta}}^{k_{2}}\right] } & =\frac{1}{\sqrt{l}} \sum_{r=0}^{l-1} \omega^{-r k_{1}}\left\langle\lambda^{r}(\beta), e_{s}\right\rangle \mathfrak{t}_{\bar{\beta}}^{k_{1}+k_{2}}, \\
{\left[\mathfrak{t}_{\frac{\alpha}{\alpha}}^{k_{1}}, \mathfrak{t}_{\bar{\beta}}^{k_{2}}\right] } & =\frac{1}{p_{\alpha} \sqrt{l}} \omega^{r k_{2}} \sum_{s}\left(\alpha^{\vee}, e_{s}\right) \mathfrak{h}_{s}^{k_{1}+k_{2}}, \quad \text { если } \alpha=-\lambda^{r}(\beta) \text { для некоторого } r .
\end{aligned}
$$

Последнее соотношение получаем из (В.5) и разложения $\mathfrak{h}_{\alpha}^{k}=\sum_{s}\left(\alpha^{\vee}, e_{s}\right) \mathfrak{h}_{s}^{k}$. Иначе те же соотношения можно записать как в (В.19)-(В.20):

$$
\begin{aligned}
{\left[\mathfrak{t}_{\alpha}^{k}, \mathfrak{t}_{\beta}^{j}\right] } & = \begin{cases}\frac{1}{\sqrt{l}} \sum_{s=0}^{l-1} \omega^{j s} C_{\alpha, \lambda^{s} \beta} \mathfrak{t}_{\alpha+\lambda^{s} \beta}^{k+j}, & \alpha \neq-\lambda^{s} \beta, \\
\frac{p_{\alpha}}{\sqrt{l}} \omega^{s j} \mathfrak{h}_{\alpha}^{k+j}, & \alpha=-\lambda^{s} \beta,\end{cases} \\
{\left[\mathfrak{h}_{\alpha}^{k}, \mathfrak{t}_{\beta}^{m}\right] } & =\frac{1}{\sqrt{l}} \sum_{s=0}^{l-1} \omega^{-k s} \frac{2\left(\alpha, \lambda^{s} \beta\right)}{(\alpha, \alpha)} \mathfrak{t}_{\beta}^{k+m}, \\
{\left[\mathfrak{H}_{\alpha}^{k}, \mathfrak{t}_{\beta}^{m}\right] } & =\frac{1}{\sqrt{l}} \sum_{s=0}^{l-1} \omega^{-k s} \frac{(\alpha, \alpha)}{2}\left(\widehat{\alpha}, \lambda^{s} \beta\right) \mathfrak{t}_{\beta}^{k+m} .
\end{aligned}
$$

\footnotetext{
${ }^{9}$ Для систем корней $A_{n}$ и $E_{6}$ канонические базисы удобно выбрать в $\mathfrak{h} \oplus \mathbb{C}$.
} 


\section{Приложение С. Эллиптические функции}

Большая часть формул и обозначений заимствована из [121], [122]. Тождества доказываются явно сравнением вычетов и квазипериодических условий на решетке $\mathbb{Z}+\tau \mathbb{Z}$.

Обозначения:

$$
\begin{gathered}
\mathbf{e}(x)=\exp (2 \pi i x), \quad q=\mathbf{e}\left(\frac{1}{2} \tau\right), \\
\omega_{1}, \omega_{2}-\text { фундаментальные полупериоды, } \quad \tau=\omega_{2} / \omega_{1} .
\end{gathered}
$$

Тэта-функиия:

$$
\begin{aligned}
\vartheta(z \mid \tau) & =\sum_{n \in \mathbb{Z}}(-1)^{n-1 / 2} \mathbf{e}\left(\frac{1}{2}\left(\left(n+\frac{1}{2}\right)^{2} \tau+(2 n+1) z\right)\right) \\
& =2 q^{1 / 4} \sum_{n=0}^{\infty}(-1)^{n} q^{n(n+1)} \sin \pi z .
\end{aligned}
$$

Функиии Эйзенштейна:

$$
\begin{aligned}
& E_{1}(z \mid \tau)=\partial_{z} \log \vartheta(z \mid \tau),\left.\quad E_{1}(z \mid \tau)\right|_{z \rightarrow 0} \sim \frac{1}{z}-2 \eta_{1} z \\
& E_{2}(z \mid \tau)=-\partial_{z} E_{1}(z \mid \tau)=\partial_{z}^{2} \log \vartheta(z \mid \tau),\left.\quad E_{2}(z \mid \tau)\right|_{z \rightarrow 0} \sim \frac{1}{z^{2}}+2 \eta_{1} .
\end{aligned}
$$

Здесь

$$
\eta_{1}(\tau)=\frac{3}{\pi^{2}} \sum_{\substack{m, n \in \mathbb{Z} \\ m^{2}+n^{2} \neq 0}} \frac{1}{(m \tau+n)^{2}}=\frac{24}{2 \pi i} \frac{\eta^{\prime}(\tau)}{\eta(\tau)}
$$

и $\eta(\tau)=q^{1 / 24} \prod_{n>0}\left(1-q^{n}\right)-$ функция Дедекинда.

Связь с функииями Вейеритрасса:

$$
\begin{aligned}
& \zeta(z, \tau)=E_{1}(z, \tau)+2 \eta_{1}(\tau) z, \\
& \wp(z, \tau)=E_{2}(z, \tau)-2 \eta_{1}(\tau) .
\end{aligned}
$$

Функиия $\phi$ :

$$
\phi(u, z)=\frac{\vartheta(u+z) \vartheta^{\prime}(0)}{\vartheta(u) \vartheta(z)} .
$$

Она имеет полюс в $z=0$ :

$$
\phi(u, z)=\frac{1}{z}+E_{1}(u)+\frac{z}{2}\left(E_{1}^{2}(u)-\wp(u)\right)+\cdots .
$$

Пусть

$$
f(u, z)=\partial_{u} \phi(u, z)=\phi(u, z)\left(E_{1}(u+z)-E_{1}(u)\right) .
$$


Формуль сложения:

$$
\begin{gathered}
\phi(u, z) f(v, z)-\phi(v, z) f(u, z)=\left(E_{2}(v)-E_{2}(z)\right) \phi(u+v, z) \\
\quad \phi(u, z) \phi(-u, z)=E_{2}(z)-E_{2}(u) \\
\phi(u, z) \phi(-u, w)=\phi(u, z-w)\left[E_{1}(u+z-w)-E_{1}(u)+E_{1}(w)-E_{1}(z)\right] . \\
\text { Пусть } \sum_{a=1}^{n} c_{a}=0 . \text { Тогда } \\
\left(\sum_{a=1}^{n} c_{a} E_{1}\left(w-x_{a}\right)\right)^{2}=\sum_{a=1}^{n}\left(c_{a}^{2} \wp\left(w-x_{a}\right)+\sum_{b \neq a} c_{a} c_{b} E_{1}\left(x_{a}-x_{b}\right) E_{1}\left(w-x_{a}\right)\right) .
\end{gathered}
$$

Уравнение теплопроводности:

$$
\partial_{\tau} \phi(u, w)-\frac{1}{2 \pi i} \partial_{u} \partial_{w} \phi(u, w)=0 .
$$

Четность:

$$
\begin{gathered}
\phi(u, z)=\phi(z, u), \quad \phi(-u,-z)=-\phi(u, z) \\
E_{1}(-z)=-E_{1}(z), \quad E_{2}(-z)=E_{2}(z) \\
f(-u,-z)=f(u, z)
\end{gathered}
$$

Квазипериодичность:

$$
\begin{aligned}
\vartheta(z+1) & =-\vartheta(z), & \vartheta(z+\tau) & =-q^{-1 / 2} e^{-2 \pi i z} \vartheta(z) ; \\
E_{1}(z+1) & =E_{1}(z), & E_{1}(z+\tau) & =E_{1}(z)-2 \pi i ; \\
E_{2}(z+1) & =E_{2}(z), & E_{2}(z+\tau) & =E_{2}(z) ; \\
\phi(u, z+1) & =\phi(u, z), & \phi(u, z+\tau) & =e^{-2 \pi i u} \phi(u, z) ; \\
f(u, z+1) & =f(u, z), & f(u, z+\tau) & =e^{-2 \pi i u} f(u, z)-2 \pi i \phi(u, z) .
\end{aligned}
$$

Основным объектом для построения операторов Лакса $r$-матриц является функция

$$
\begin{aligned}
& \varphi_{\alpha}^{k}(\mathbf{u}, z)=e^{2 \pi i\langle\varrho, \alpha\rangle z} \phi\left(\langle\mathbf{u}+\varrho \tau, \alpha\rangle+\frac{k}{l}, z\right) \quad\left(\varrho=\frac{\rho}{h}\right), \\
& \varphi_{0}^{k}(\mathbf{u}, z)=\phi\left(\frac{k}{l}, z\right), \\
& \varphi_{0}^{0}(\mathbf{u}, z)=E_{1}(z)
\end{aligned}
$$

и

$$
\begin{aligned}
& f_{\alpha}^{k}(\mathbf{u}, z)=e^{2 \pi i\langle\varrho, \alpha\rangle z} f\left(\langle\mathbf{u}+\varrho \tau, \alpha\rangle+\frac{k}{l}, z\right) \\
& f_{\alpha}^{k}(\mathbf{u}, 0)=-E_{2}\left(\langle\mathbf{u}+\varrho \tau, \alpha\rangle+\frac{k}{l}\right)=-\wp\left(\langle\mathbf{u}+\varrho \tau, \alpha\rangle+\frac{k}{l}\right)-2 \eta_{1}, \\
& f_{0}^{0}(\mathbf{u}, z)=f_{0}^{0}(z)=\frac{1}{2}\left(E_{1}^{2}(z)-\wp(z)\right)
\end{aligned}
$$


Уравнение теплопроводности принимает вид

$$
2 \pi i \partial_{\tau} \varphi_{\alpha}^{k}(\mathbf{u}, z)=\partial_{z} f_{\alpha}^{k}(\mathbf{u}, z) .
$$

Для экономности будем опускать иногда зависимость от $u$.

Тождества Фэя:

$$
\phi\left(u_{1}, z_{1}\right) \phi\left(u_{2}, z_{2}\right)-\phi\left(u_{1}+u_{2}, z_{1}\right) \phi\left(u_{2}, z_{2}-z_{1}\right)-\phi\left(u_{1}+u_{2}, z_{2}\right) \phi\left(u_{1}, z_{1}-z_{2}\right)=0 \text {. }
$$

Дифференцируя по $u_{2}$, находим:

$$
\begin{aligned}
& \phi\left(u_{1}, z_{1}\right) f\left(u_{2}, z_{2}\right)-\phi\left(u_{1}+u_{2}, z_{1}\right) f\left(u_{2}, z_{2}-z_{1}\right) \\
& \quad=\phi\left(u_{2}, z_{2}-z_{1}\right) f\left(u_{1}+u_{2}, z_{1}\right)+\phi\left(u_{1}, z_{1}-z_{2}\right) f\left(u_{1}+u_{2}, z_{2}\right) .
\end{aligned}
$$

Подставляя сюда

$$
\begin{array}{ll}
u_{1}=\langle u+\rho \tau, \alpha+\beta\rangle+\frac{k+m}{l}, & u_{2}=-\langle u+\rho \tau, \beta\rangle-\frac{m}{l}, \\
z_{1}=z_{a}-z_{c}=z_{a c}, & z_{2}=z_{b}-z_{c}=z_{b c}
\end{array}
$$

и умножая на подходящий экспоненциальный множитель, получим

$$
\varphi_{\alpha}^{k}\left(z_{a c}\right) f_{\beta}^{m}\left(z_{a b}\right)-\varphi_{\beta}^{m}\left(z_{a b}\right) f_{\alpha}^{k}\left(z_{a c}\right)+\varphi_{\alpha+\beta}^{k+m}\left(z_{a b}\right) f_{\alpha}^{k}\left(z_{c b}\right)-\varphi_{\alpha+\beta}^{k+m}\left(z_{a c}\right) f_{-\beta}^{-m}\left(z_{b c}\right)=0 .
$$

Полагая $m=0, \beta=0$ и используя разложение

$$
\phi(u, z) \sim \frac{1}{u}+E_{1}(z)+u f_{0}^{0}(z)+\cdots,
$$

находим:

$$
\varphi_{\alpha}^{k}\left(z_{a c}\right) f_{0}^{0}\left(z_{a b}\right)-E_{1}\left(z_{a b}\right) f_{\alpha}^{k}\left(z_{a c}\right)+\varphi_{\alpha}^{k}\left(z_{a b}\right) f_{\alpha}^{k}\left(z_{b c}\right)-\varphi_{\alpha}^{k}\left(z_{a c}\right) f_{0}^{0}\left(z_{c b}\right)=\frac{1}{2} \partial_{u} f_{\alpha}^{k}\left(z_{a c}\right) .
$$

Еще тождества Фэя:

$$
\begin{aligned}
\varphi_{\alpha}^{k}\left(z_{a c}\right) f_{\beta}^{m}\left(z_{a c}\right)-\varphi_{\beta}^{m}\left(z_{a c}\right) f_{\alpha}^{k}\left(z_{a c}\right) & =\varphi_{\alpha+\beta}^{k+m}\left(z_{a c}\right)\left(\wp_{\alpha}^{k}-\wp_{\beta}^{m}\right), \\
\varphi_{\beta}^{m}\left(z_{a c}\right) f_{-\beta}^{-m}\left(z_{a c}\right)-\varphi_{-\beta}^{-m}\left(z_{a c}\right) f_{\beta}^{m}\left(z_{a c}\right) & =E_{2 \beta}^{\prime m}, \\
\varphi_{\beta}^{k}\left(z_{a c}\right) \wp_{\beta}^{k}-\varphi_{\beta}^{k}\left(z_{a c}\right) f_{0}^{0}\left(z_{a c}\right)+E_{1}\left(z_{a c}\right) f_{\beta}^{k}\left(z_{a c}\right) & =\frac{1}{2} \partial_{u} f_{\beta}^{k}\left(z_{a c}\right) .
\end{aligned}
$$

Последнее следует из

$$
\partial_{u} \phi(u, z)=\phi(u, z)\left(E_{1}(z+u)-E_{1}(u)\right)
$$

и

$$
\left(E_{1}(z+u)-E_{1}(u)-E_{1}(z)\right)^{2}=\wp(z)+\wp(u)+\wp(z+u) .
$$

Модулярнве свойства. Пусть $\left(\begin{array}{ll}a & b \\ c & d\end{array}\right) \in \mathrm{SL}(2, \mathbb{Z})$, т. е. $a, b, c, d \in \mathbb{Z}$ и $\mathrm{ad}-b c=1$. Тогда

$$
\theta\left(\frac{z}{c \tau+d} \mid \frac{a \tau+b}{c \tau+d}\right)=\varepsilon e^{\pi i / 4}(c \tau+d)^{1 / 2} \exp \left(\frac{i \pi c z^{2}}{c \tau+d}\right) \theta(z \mid \tau) \quad\left(\varepsilon^{8}=1\right)
$$




$$
\begin{gathered}
E_{1}\left(\frac{z}{c \tau+d} \mid \frac{a \tau+b}{c \tau+d}\right)=(c \tau+d) E_{1}(z \mid \tau)+2 \pi i c z, \\
E_{2}\left(\frac{z}{c \tau+d} \mid \frac{a \tau+b}{c \tau+d}\right)=(c \tau+d)^{2} E_{2}(z \mid \tau)-2 \pi i c(c \tau+d), \\
\phi\left(\frac{u}{c \tau+d}, \frac{z}{c \tau+d} \mid \frac{a \tau+b}{c \tau+d}\right)=(c \tau+d) \exp \left(2 \pi i \frac{c z u}{c \tau+d}\right) \phi(u, z \mid \tau) .
\end{gathered}
$$

\section{Приложение D. Характеристические классы и конформные группы}

Здесь опишем конструкцию конформных групп, предложенную в [45].

Конформные группы. Наша цель состоит в определении аналога группы $\mathrm{GL}(N, \mathbb{C})$ для групп, отличных от $\mathrm{SL}(N, \mathbb{C})$. Пусть

$$
\phi: \mathscr{Z}(\bar{G}) \hookrightarrow\left(\mathbb{C}^{*}\right)^{r}
$$

- вложение центра $\mathscr{Z}(\bar{G})$ в алгебраический тор $\left(\mathbb{C}^{*}\right)^{r}$ минимальной размерности $\left(r=1\right.$ для циклического центра и $r=2$ в случае $\left.\mu_{2} \times \mu_{2}\right)$. Заметим, что любые два вложения $\mathscr{Z}(\bar{G})(\bar{G} \neq \mathrm{SL}(N, \mathbb{C}))$ сопряжены слева: $\phi_{1}=A \phi_{2}$ для некоторого автоморфизма $A$ тора $\left(\mathbb{C}^{*}\right)^{r}$. В нашем случае имеем группы $\mu_{2}$, $\mu_{3}, \mu_{4}$ или $\mu_{2} \times \mu_{2}$. В этих случаях нетривиальные корни из единицы либо совпадают, либо обратны друг другу. В последнем случае автоморфизм $A$ имеет вид $x \rightarrow x^{-1}$.

Рассмотрим "антидиагональное" вложение $\mathscr{Z}(\bar{G}) \rightarrow \bar{G} \times\left(\mathbb{C}^{*}\right)^{r}, \zeta \mapsto\left(\zeta, \phi(\zeta)^{-1}\right)$, $\zeta \in \mathscr{Z}(\bar{G})$. Образ этого отображения есть нормальная подгруппа, так как $\mathscr{Z}-$ центр $\bar{G}$.

ОПРЕДЕЛЕНИЕ D.1. Фактор

$$
C \bar{G}=\left(\bar{G} \times\left(\mathbb{C}^{*}\right)^{r}\right) / \mathscr{Z}(\bar{G})
$$

называется конформной версией $\bar{G}$.

Аналогичным образом конформная версия может быть определена для любой $G$ с нетривиальным центром. Если центр $G$ тривиален, как для $G^{\text {ad }}$, то $C G=G \times \mathbb{C}^{*}$.

Группа $C \bar{G}$ не зависит от способа вложения в $\mathbb{C}^{r}$ вследствие замечания о сопряженности $\phi$. Имеется естественное вложение $\bar{G} \subset C \bar{G}$. Рассмотрим фактортор $Z^{\vee}=\left(\mathbb{C}^{*}\right)^{r} / \mathscr{Z}(\bar{G}) \sim\left(\mathbb{C}^{*}\right)^{r}$. Последний изоморфизм задается как $\lambda \rightarrow \lambda^{N}$ для циклического центра и $\left(\lambda_{1}, \lambda_{2}\right) \rightarrow\left(\lambda_{1}^{2}, \lambda_{2}^{2}\right)$ для $D_{\text {even. Последовательность }}$

$$
1 \rightarrow \bar{G} \rightarrow C \bar{G} \rightarrow Z^{\vee} \rightarrow 1
$$

есть аналог последовательности

$$
1 \rightarrow \mathrm{SL}(N, \mathbb{C}) \rightarrow \mathrm{GL}(N, \mathbb{C}) \rightarrow \mathbb{C}^{*} \rightarrow 1
$$

$\mathrm{C}$ другой стороны, имеем вложение $\left(\mathbb{C}^{*}\right)^{r} \rightarrow C \bar{G}$ с фактором $C \bar{G} /\left(\mathbb{C}^{*}\right)^{r}=G^{\mathrm{ad}}$. Тогда последовательность

$$
1 \rightarrow\left(\mathbb{C}^{*}\right)^{r} \rightarrow C \bar{G} \rightarrow G^{\text {ad }} \rightarrow 1
$$


аналогична последовательности

$$
1 \rightarrow \mathbb{C}^{*} \rightarrow \operatorname{GL}(N, \mathbb{C}) \rightarrow \operatorname{PGL}(N, \mathbb{C}) \rightarrow 1
$$

Пусть $\pi$ - неприводимое представление $\bar{G}$, а $\chi$ - характер тора $\left(\mathbb{C}^{*}\right)^{r}$. Из (D.2) следует, что неприводимое представление $\widetilde{\pi}$ группы $C \bar{G}$ определяется следующим образом:

$$
\tilde{\pi}=\pi \otimes \chi\left(\left(\mathbb{C}^{*}\right)^{r}\right) \quad \text { с условием }\left.\quad \pi\right|_{\mathscr{Z}(\bar{G})}=\chi \phi \quad(\phi \text { определено в }(\mathrm{D} \cdot 1)) .
$$

Предположим для простоты, что $\pi$ - фундаментальное представление. Это означает, что его старший вес $\nu$ - это фундаментальный вес. Пусть $\varpi^{\vee}-$ фундаментальный ковес, генерирующий $\mathscr{Z}(\bar{G})$ для $r=1$. Иными словами, $\zeta=\mathbf{e}\left(\varpi^{\vee}\right)$ - генератор $\mathscr{Z}(\bar{G})\left(\zeta^{N}=1, N=\operatorname{ord}(\mathscr{Z}(\bar{G}))\right)$. Тогда $\left.\pi\right|_{\mathscr{Z}(\bar{G})}$ действует скаляром $\mathbf{e}\left(\left\langle\varpi^{\vee}, \nu\right\rangle\right)$. Старший вес разлагается по базису простых корней: $\nu=$ $\sum_{\alpha \in \Pi} c_{\alpha}^{\nu} \alpha$. Тогда коэффициенты $c_{\alpha}^{\nu}-$ строки обратной матрицы Картана. Эти коэффициенты имеют вид $k / N$ с целым $k$. Следовательно, скаляр

$$
\mathbf{e}\left(\left\langle\varpi^{\vee}, \nu\right\rangle\right)=\mathbf{e}\left(\sum_{\alpha \in \Pi} c_{\alpha}^{\nu} \delta_{\langle\varpi \vee, \alpha\rangle}\right)
$$

является корнем из единицы. С другой стороны, пусть $\chi_{m}\left(\mathbb{C}^{*}\right)=w^{m}\left(w \in \mathbb{C}^{*}\right)-$ характер $\mathbb{C}^{*}$ и $\phi(\zeta)=\mathbf{e}(l / N)$. В терминах весов определение $\widetilde{\pi}(\mathrm{D} .4)$ принимает вид $\mathbf{e}\left(\left\langle\varpi^{\vee}, \nu\right\rangle\right)=\mathbf{e}(m l / N)$. Из этой конструкции следует, что характеры группы $C \bar{G}$ определяются решеткой весов $P$ и целочисленной решеткой $\mathbb{Z}$ с условием $\chi_{(\gamma, m)}(\mathbf{x}, w)=\exp (2 \pi i\langle\gamma, \mathbf{x}\rangle) w^{m}, \quad\left\langle\gamma, \varpi^{\vee}\right\rangle=\frac{m l}{N}+j, \quad \gamma \in P, \quad m, j \in \mathbb{Z}, \quad \mathbf{x} \in \mathfrak{h}$. Случай $D_{\text {even }}$ (например, $r=2$ ) рассматривается аналогично.

ЗАмЕчАнИЕ D.1. Простые группы могут быть определены как подгруппы GL $(V)$, сохраняющие некоторые мультилинейные формы в $V$. Например, в определяемых представлениях это билинейные симметрические формы для SO, билинейные антисимметрические формы $\mathrm{Sp}$, трилинейные формы для $E_{6}$ и формы четвертого порядка для $E_{7}$. В общей ситуации $G$ определяется как подгруппа в $\mathrm{GL}(V)$, сохраняющая три-тензор в $V^{*} \otimes V^{*} \otimes V$ [123]. Конформные версии этих групп могут быть иначе определены как преобразования, сохраняющие формы с точностью до дилатаций. Мы предпочитаем использовать алгебраическую конструкцию, что и оправдывает термин "конформные версии”.

Конформные версии можно определить и через точные представления $\bar{G}$. Пусть $V$ является таким представлением, и предположим, что $\mathscr{Z}(\bar{G})$ - циклическая группа. Тогда $C \bar{G}$ - подгруппа в $\mathrm{GL}(V)$, генерируемая $G$ и дилатациями $\mathbb{C}^{*}$. Характер $\operatorname{det} V$ равен $\lambda^{\operatorname{dim} V}$, где $\lambda$ равна (D.5) для фундаментальных представлений.

Для $D_{\text {even }}$ используем два представления, например левых и правых спиноров $\operatorname{Spin}^{\mathrm{L}, \mathrm{R}}$. Конформная группа $C \operatorname{Spin}_{4 k}-$ подгруппа в группе $\mathrm{GL}\left(\operatorname{Spin}^{\mathrm{L}} \oplus \operatorname{Spin}^{\mathrm{R}}\right)$, генерируемой $\operatorname{Spin}_{4 k}$ и $\mathbb{C}^{*} \times \mathbb{C}^{*}$, где первый множитель $\mathbb{C}^{*}$ действует дилатациями на $\operatorname{Spin}^{\mathrm{L}}$, а второй - на $\operatorname{Spin}^{\mathrm{R}}$. Характеры $\operatorname{det} \operatorname{Spin}^{\mathrm{L}}$ и $\operatorname{det} \operatorname{Spin}^{\mathrm{R}}$ равны $\lambda_{1}^{\operatorname{dim}\left(\operatorname{Spin}_{4 k}^{\mathrm{L}}\right)}$ и $\lambda_{2}^{\operatorname{dim}\left(\operatorname{Spin}_{4 k}^{\mathrm{R}}\right)}$ соответственно $\left(\operatorname{dim}\left(\operatorname{Spin}_{4 k}^{\mathrm{L}, \mathrm{R}}\right)=2^{2 k-1}\right)$. 
Характеристические классы и степени векторных расслоений. Из точной последовательности (D.3) и зануления вторых когомологий (кривой) $H^{2}\left(\Sigma, \mathscr{O}^{*}\right)=0$ с коэффициентами в аналитических пучках, получаем, что любое $G^{\mathrm{ad}}(\mathscr{O})$-расслоение (даже топологически нетривиальное с $\zeta\left(G^{\mathrm{ad}}(\mathscr{O})\right) \neq 0$ ) можно поднять до $C \bar{G}(\mathscr{O})$-расслоения.

Пусть $V$ - точное представление (неприводимое, либо сумма $\mathrm{Spin}^{\mathrm{L}} \oplus \mathrm{Spin}^{\mathrm{R}}$ для $\left.D_{2 k}\right)$. Тогда из (D.1) имеем вложение $\mathscr{Z}(\bar{G})$ в автоморфизмы $V$ :

$$
\phi_{V}: \mathscr{Z}(\bar{G}) \hookrightarrow\left(\mathbb{C}^{*}\right)^{r}=\operatorname{Aut}_{\bar{G}}(V) .
$$

В частном случае, когда $V$ - фундаментальное представление, центр действует умножением на (D.5). Пусть $\mathscr{P}_{C \bar{G}}$ - главное $C \bar{G}(\mathscr{O})$-расслоение. Обозначим через $E(V)=\mathscr{P}_{C \bar{G}} \otimes_{C \bar{G}} V$ (или $\left.E\left(\operatorname{Spin}^{\mathrm{L}, \mathrm{R}}\right)\right)$ векторное расслоение, индуцированное $V\left(\operatorname{Spin}^{\mathrm{L}, \mathrm{R}}\right.$ для $\left.D_{\text {even }}\right)$.

Tеорема D.1. Пусть $E_{\mathrm{ad}}=E(\mathrm{Ad})$ - присоединенное расслоение с характеристическим классом $\zeta\left(E_{\mathrm{ad}}\right)$. Образ $\zeta\left(E_{\mathrm{ad}}\right)$ под действием $\phi_{V}$ (D.6) есть

$$
\phi_{V}\left(\zeta\left(E_{\mathrm{ad}}\right)\right)= \begin{cases}\exp \left(-\frac{2 \pi i \operatorname{deg}\left(E_{\bar{G}}(V)\right)}{\operatorname{dim} V}\right), & \mathscr{Z}(\bar{G}) \text { ииклична, } \\ \exp \left(-\frac{2 \pi i \operatorname{deg}\left(E_{\operatorname{Spin}_{4 k}^{\mathrm{L}, \mathrm{R}}}\right)}{2^{2 k-1}}\right) .\end{cases}
$$

Для $G=\mathrm{GL}(N, \mathbb{C})$ эта теорема была доказана в [82].

ДокАЗАТЕЛЬство. Рассмотрим коммутативную диаграмму

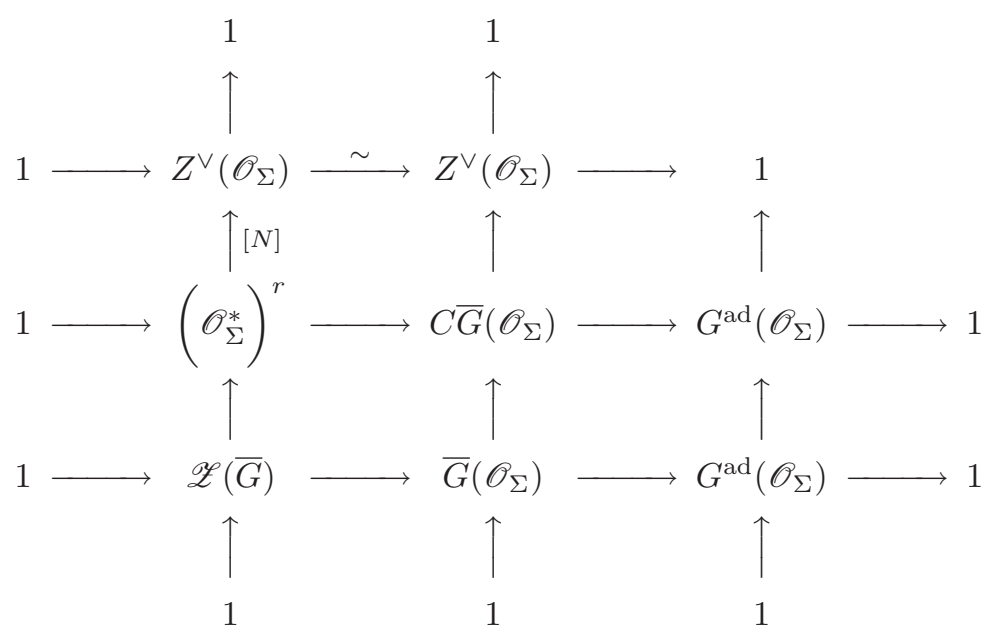

и соответствующую диаграмму из чеховских коцепей. Пусть $\psi$ есть 1-коцикл со значениями в $G^{\text {ad }}\left(\mathscr{O}_{\Sigma}\right)$. Рассмотрим его прообраз как коцикл со значениями в $C \bar{G}\left(\mathscr{O}_{\Sigma}\right)$. Вследствие определения $C \bar{G}$ этот коцикл есть пара коцепей $(\Psi, \nu)$ со значениями в $\bar{G}\left(\mathscr{O}_{\Sigma}\right)$ и $\left(\mathscr{O}_{\Sigma}^{*}\right)^{r}$ таких, что $\phi_{V}(d \Psi) d \nu=1 \in\left(\mathscr{O}^{*}\right)^{r}$, где $d$ - чеховский кограничный оператор. Класс когомологий $d \Psi$ по опре- 
делению есть характеристический класс с, т. е. $\phi_{V}$ от него обратно классу $d \nu$ : $\phi_{V}\left(\zeta\left(E_{\mathrm{ad}}\right)\right)=(d \nu)^{-1}$. Так как $\nu$ действует в $V$ скаляром $\nu^{\operatorname{dim}(V)}$, это 1-коцикл, как и детерминант. Он представляет собой детерминант линейного расслоения $E(V)$. Таким образом, $\nu$ есть прообраз коцикла $\nu^{\operatorname{dim}(V)}$ при отображении $N=\operatorname{dim}(V)$-й степени $\mathscr{O}^{*} \stackrel{[N]}{\longrightarrow} \mathscr{O}^{*}, \nu \rightarrow \nu^{N}, N=\operatorname{dim}(V)$.

Рассмотрим длинную точную последовательность

$$
1 \longrightarrow \mu_{N} \longrightarrow \mathscr{O}_{\Sigma}^{*} \stackrel{[N]}{\longrightarrow} \mathscr{O}_{\Sigma}^{*} \longrightarrow 1 \quad\left(\mu_{N}=\mathbb{Z} / N \mathbb{Z}\right) .
$$

Она индуцирует отображение $H^{1}\left(\Sigma, \mathscr{O}_{\Sigma}^{*}\right) \rightarrow H^{2}\left(\Sigma, \mu_{N}\right)$. Коцикл $d \nu$ лежит в когомологическом классе, который есть образ класса $\operatorname{det} E(V)=\nu^{N}$ при кограничном отображении $H^{1}\left(\Sigma, \mathscr{O}^{*}\right) \rightarrow H^{2}\left(\Sigma, \mu_{N}\right)$. Обозначим его через $\operatorname{Inv}_{N}=$ Image $(\operatorname{det} E(V))$. Тогда по определению получаем, что класс $d \nu$ получается

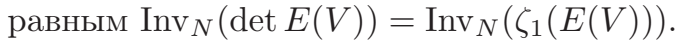

Утверждение теоремы вытекает из следующего предложения.

ПреДЛОЖеНИЕ D.1. Пусть $\gamma$ есть 1-коцикл со значениями в $\mathscr{O}^{*}$. Тогда $\operatorname{Inv}_{N}(\gamma)=\exp ((1 / N) \cdot 2 \pi i \operatorname{deg}(\gamma))$.

ДокАЗАТЕЛЬСтво. Рассмотрим диаграмму

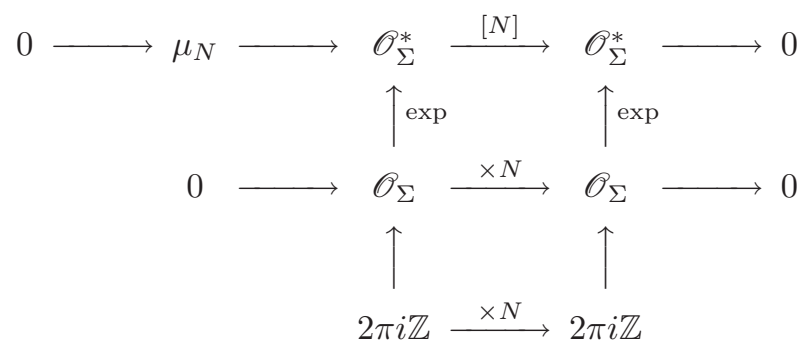

Пусть $\gamma$ есть 1-коцикл в $\mathscr{O}_{\Sigma}^{*}$. По определению его образ в $H^{2}\left(X, \mu_{N}\right)$ есть кограница 1-коцепи $\gamma^{1 / N}$ от $\mathscr{O}_{\Sigma}^{*},\left(\gamma^{1 / N}\right)^{N}=\gamma$. Пусть $\log (\gamma)$ есть прообраз цикла $\gamma$ при экспоненциальном отображении; $\log (\gamma)$ есть 1 -коцепь от $\mathscr{O}_{\Sigma}$, и его кограница равна степени $\gamma$, умноженной на $2 \pi i$. Так как умножение на $N$ обратимо на $\mathscr{O}_{\Sigma}$, то коцепь $(1 / N) \log (\gamma)$ корректно определена. Вследствие коммутативности диаграммы, $\exp ((1 / N) \log (\gamma))$ можно выбрать в виде $\gamma^{1 / N}$. Тем самым, образ $\gamma$ в $H^{2}\left(X, \mu_{N}\right)$ равен когранице $\exp ((1 / N) \log (\gamma))$.

Случай $r=2$ можно рассмотреть аналогичным образом. Теорема D.1 доказана.

Пусть, как и выше, $\varpi^{\vee}-$ фундаментальный ковес, генерирующий центр $\mathscr{Z}(\bar{G})$, а $\nu$ - вес представления $\bar{G}$ в $V$. Тогда из теоремы D.1 и (D.5) следует, что

$$
\operatorname{deg} E(V)=\operatorname{dim}(V)\left(\left\langle\varpi^{\vee}, \nu\right\rangle+k\right), \quad k \in \mathbb{Z} .
$$

Из нашего рассмотрения следует, что замена матрицы перехода

$$
\Lambda \rightarrow \widetilde{\Lambda}(z)=\mathbf{e}\left(\left\langle\varpi^{\vee}, \nu\right\rangle\left(z+\frac{\tau}{2}\right)\right) \Lambda
$$


ТАБлицА 5. Степени расслоений конформных групп. $\mathrm{Mp}_{n}(\mathbb{C})-$ универсальная накрывающая $\operatorname{Sp}_{n}(\mathbb{C})$.

\begin{tabular}{|c|c|c|c|}
\hline $\bar{G}$ & $\nu$ & $V$ & $\operatorname{deg}(E(V))$ \\
\hline $\operatorname{SL}(n, \mathbb{C})$ & $\varpi_{1}^{\vee}$ & $\underline{n}$ & $-1+k n$ \\
$\operatorname{Spin}_{2 n+1}(\mathbb{C})$ & $\varpi_{n}^{\vee}$ & $\underline{2^{n}}$ & $2^{n-1}(1+2 k)$ \\
$\operatorname{Mp}_{n}(\mathbb{C})$ & $\varpi_{1}^{\vee}$ & $\underline{2 n}$ & $n(1+2 k)$ \\
$\operatorname{Spin}_{4 n}^{\mathrm{L}, \mathrm{R}}(\mathbb{C})$ & $\varpi_{n, n-1}^{\vee}$ & $\underline{2^{2 n-1}}$ & $2^{2 n-2}(1+2 k)$ \\
$\operatorname{Spin}_{4 n+2}(\mathbb{C})$ & $\varpi_{n}^{\vee}$ & $\underline{2^{n}}$ & $2^{n-2}(1+4 k)$ \\
$E_{6}(\mathbb{C})$ & $\varpi_{1}^{\vee}$ & $\underline{27}$ & $9(1+3 k)$ \\
$E_{7}(\mathbb{C})$ & $\varpi_{1}^{\vee}$ & $\underline{56}$ & $28(1+2 k)$ \\
\hline
\end{tabular}

$(k \in \mathbb{Z})$

определяет расслоение конформной группы $C G$ степени (D.7). Для фундаментальных представлений $\bar{G}$ формула (D.7) проиллюстрирована в табл. 5 .

\section{Список литературы}

[1] P. Painlevé, "Mémoire sur les équations différentielles dont l'intégrale générale est uniforme", Bull. Soc. Math. France, 28 (1900), 201-261.

[2] P. Painlevé, "Sur les équations différentielles du second ordre et d'ordre supérieur dont l'intégrale générale est uniforme", Acta Math., 25:1 (1902), 1-85.

[3] B. Gambier, "Sur les équations différentielles du second ordre et du premier degré dont l'intégrale générale est à points critique fixes", Acta Math., 33:1 (1910), 1-55.

[4] R. Fuchs, "Über lineare homogene Differentialgleichungen zweiter Ordnung mit drei im Endlichen gelegenen wesentlich singulären Stellen", Math. Ann., 63:3 (1907), 301-321.

[5] R. Garnier, "Sur des équations différentielles du troisième ordre dont l'intégrale générale est uniforme et sur une classe d'équations nouvelles d'ordre supérieur dont l'intégrale générale a ses points critique fixés", Ann. Sci. École Norm. Sup. (3), 29 (1912), 1-126.

[6] L. Schlesinger, "Über eine Klasse von Differentialsystemen beliebiger Ordnung mit festen kritischen Punkten", J. Reine Angew. Math., 141 (1912), 96-145.

[7] H. Flaschka, A. C. Newell, "Monodromy- and spectrum-preserving deformations. I", Comm. Math. Phys., 76:1 (1980), 65-116.

[8] H. Flaschka, A.C. Newell, "Multiphase similarity solutions of integrable evolution equations", Phys. D, 3:1-2 (1981), 203-221.

[9] M. Jimbo, T. Miwa, K. Ueno, "Monodromy preserving deformation of linear ordinary differential equations with rational coefficients: I. General theory and $\tau$-function", Phys. D, 2:2 (1981), 306-352.

[10] M. Jimbo, T. Miwa, "Monodromy preserving deformation of linear ordinary differential equations with rational coefficients. II", Phys. D, 2:3 (1981), 407-448.

[11] M. Jimbo, T. Miwa, "Monodromy preserving deformation of linear ordinary differential equations with rational coefficients. III", Phys. D, 4:1 (1981), 26-46. 
[12] E. L. Ince, Ordinary differential equations, Dover Publications, New York, 1956, viii+558 pp.

[13] K. Iwasaki, H. Kimura, S. Shimomura, M. Yoshida, From Gauss to Painlevé. A modern theory of special functions, Aspects Math., E16, Friedr. Vieweg \& Sohn, Braunschweig, 1991, xii+347 pp.

[14] The Painlevé property. One century later, CRM Ser. Math. Phys., ed. R. Conte, Springer-Verlag, New York, 1999, xxvi+810 pp.

[15] A. R. Its, V. Yu. Novokshenov, The isomonodromic deformation method in the theory of Painlevé equations, Lecture Notes in Math., 1191, Springer-Verlag, Berlin, 1986, iv $+313 \mathrm{pp}$.

[16] A. S. Fokas, A.R. Its, A. A. Kapaev, V. Yu. Novokshenov, Painlevé transcendents. The Riemann-Hilbert approach, Math. Surveys Monogr., 128, Amer. Math. Soc., Providence, RI, 2006, xii+553 pp.

[17] Painlevé transcedents. Their asymptotics and physical applications, Proceedings of the NATO Advanced Research Workshop (Sainte-Adèle, Canada, 1990), NATO Adv. Sci. Inst. Ser. B: Phys., 278, eds. D. Levi, P. Winternitz, Springer-Verlag, New York, NY, 1992, xxvi+446 pp.

[18] B. Dubrovin, "Geometry of 2D topological field theories", Integrable systems and quantum groups (Montecatini Terme, 1993), Lecture Notes in Math., 1620, Springer, Berlin, 1996, 120-348.

[19] А. А. Болибрух, Фуксовы дифференциальные уравнения и голоморфные расслоения, МЦНМО, М., 2000, 127 с.

[20] A. A. Bolibruch, "On isomonodromic deformations of Fuchsian systems", J. Dynam. Control Syst., 3:4 (1997), 589-604.

[21] S. Yu. Slavyanov, W. Lay, Special functions. A unified theory based on singularities, with a foreword by A. Seeger, Oxford Math. Monogr., Oxford Univ. Press, Oxford, 2000, xvi+293 pp.

[22] I. Krichever, "Isomonodromy equations on algebraic curves, canonical transformations and Whitham equations", Mosc. Math. J., 2:4 (2002), 717-752.

[23] P. Boalch, "From Klein to Painlevé via Fourier, Laplace and Jimbo", Proc. London Math. Soc. (3), 90:1 (2005), 167-208.

[24] Р. Р. Гонцов, В. А. Побережный, Г. Ф. Хельминк, “Деформации систем линейных дифференциальных уравнений”, УМН, 66:1(397) (2011), 65-110; англ. пер.: R. R. Gontsov, V.A. Poberezhnyi, G.F. Khel'minck, "On deformations of linear differential systems", Russian Math. Surveys, 66:1 (2011), 63-105.

[25] K. Okamoto, "On Fuchs' problem on a torus", Japan-United States seminar on ordinary differential and functional equations (Kyoto, 1971), Lecture Notes in Math., 243, Springer, Berlin, 1971, 277-280.

[26] K. Iwasaki, "Moduli and deformation for Fuchsian projective connections on a Riemann surface", J. Fac. Sci. Univ. Tokyo Sect. IA Math., 38:3 (1991), 431-531.

[27] S. Kawai, Deformation of complex structures on a torus and monodromy preserving deformation, preprint, University of Tokyo, 1995.

[28] A. M. Levin, M.A. Olshanetsky, Classical limit of the Knizhnik-ZamolodchikovBernard equations as hierarchy of isomonodromic deformations. Free fields approach, 1997 (v3 - 1998), 40 pp., arXiv: hep-th/9709207.

[29] M. A. Olshanetsky, "Generalized Hitchin systems and the Knizhnik-ZamolodchikovBernard equation on ellipic curves", Lett. Math. Phys., 42:1 (1997), 59-71; 1995, 11 pp., arXiv: hep-th/9510143. 
[30] A. Levin, M. Olshanetsky, "Hierarchies of isomonodromic deformations and Hitchin systems", Moscow seminar in mathematical physics, Amer. Math. Soc. Transl. Ser. 2, 191, Amer. Math. Soc., Providence, RI, 1999, 223-262.

[31] N. Hitchin, "Stable bundles and integrable systems", Duke Math. J., 54:1 (1987), 91-114.

[32] N. Nekrasov, "Holomorphic bundles and many-body systems", Comm. Math. Phys., 180:3 (1996), 587-603; 1995, 21 pp., arXiv: hep-th/9503157.

[33] A. Gorsky, N. Nekrasov, Elliptic Calogero-Moser system from two dimensional current algebra, 1994, 8 pp., arXiv: hep-th/9401021.

[34] B. Enriquez, V. Rubtsov, "Hitchin systems, higher Gaudin operators and $r$-matrices", Math. Res. Lett., 3:3 (1996), 343-357.

[35] A. Levin, M. Olshanetsky, "Double coset construction of moduli space of holomorphic bundles and Hitchin systems", Commun. Math. Phys., 188:2 (1997), 449-466; 1996, 19 pp., arXiv: alg-geom/9605005.

[36] D. Korotkin, H. Samtleben, "On the quantization of isomonodromic deformations on the torus", Internat. J. Modern Phys. A, 12:11 (1997), 2013-2029.

[37] G. Kuroki, T. Takebe, "Twisted Wess-Zumino-Witten models on elliptic curves", Comm. Math. Phys., 190:1 (1997), 1-56; 1996 (v4 - 1997), 55 pp., arXiv: q-alg/ 9612033.

[38] K. Takasaki, "Gaudin model, KZ equation, and isomonodromic problem on torus", Lett. Math. Phys., 44:2 (1998), 143-156; 1997, 15 pp., arXiv: hep-th/9711058.

[39] J. Harnad, M. A. Wisse, "Loop algebra moment maps and Hamiltonian models for the Painlevé transcendants", Mechanics day (Waterloo, ON, 1992), Fields Inst. Commun., 7, Amer. Math. Soc., Providence, RI, 1996, 155-169; 1993, 14 pp., arXiv: hep-th/9305027.

[40] А.Г. Рейман, М.А. Семенов-Тян-Шанский, “Алгебры Ли и лаксовы уравнения со спектральным параметром на эллиптической кривой”, Вопросы квантовой теории поля и статистической физики. 6, Зап. науч. сем. ЛОМИ, 150, Изд-во "Наука", Ленинград. отд., Л., 1986, 104-118; англ. пер.: А. G. Reiman, M. A. Semenov-Tian-Schanskii, "Lie algebras and Lax equations with spectral parameter on elliptic curve", J. Soviet Math., 46:1 (1989), 1631-1640.

[41] И. М. Кричевер, "Эллиптические решения уравнения Кадомцева-Петвиашвили и интегрируемые системы частиц", Функи. анализ и его прил., 14:4 (1980), 45-54; англ. пер.: I. M. Krichever, "Elliptic solutions of the Kadomtsev-Petviashvili equation and integrable systems of particles", Funct. Anal. Appl., 14:4 (1980), 282-290.

[42] Yu. Chernyakov, A. M. Levin, M. Olshanetsky, A. Zotov, "Elliptic Schlesinger system and Painlevé VI", J. Phys. A, 39:39 (2006), 12083-12101; 2006, 16 pp., arXiv: nlin/0602043.

[43] M. A. Olshanetsky, A. V. Zotov, "Isomonodromic problems on elliptic curve, rigid tops and reflection equations", Elliptic integrable systems, Rokko Lectures in Math., 18, Kobe Univ., Kobe, Japan, 2005, 149-171.

[44] A. M. Levin, M. A. Olshanetsky, "Painlevé-Calogero correspondence", CalogeroMoser-Sutherland models (Montréal, QC, 1997), CRM Ser. Math. Phys., Springer, New York, 2000, 313-332; 1997, 17 pp., arXiv: alg-geom/9706010.

[45] A. Levin, M. Olshanetsky, A. Smirnov, A. Zotov, "Characteristic classes and Hitchin systems. General construction", Comm. Math. Phys., 316:1 (2012), 1-44; Characteristic classes and integrable systems. General construction, 2010, 52 pp., arXiv: 1006.0702 . 
[46] A. Levin, M. Olshanetsky, A. Smirnov, A. Zotov, "Calogero-Moser systems for simple Lie groups and characteristic classes of bundles", J. Geom. Phys., 62:8 (2012), 1810-1850; Characteristic classes and integrable systems for simple Lie groups, 2010, 51 pp., arXiv: 1007.4127.

[47] А.В. Зотов, А.В. Смирнов, "Модификации расслоений, эллиптические интегрируемые системы и связанные задачи”, ТМФ, 177:1 (2013), 3-67; англ. пер.: A. V. Zotov, A. V. Smirnov, "Modifications of bundles, elliptic integrable systems, and related problems", Theoret. and Math. Phys., 177:1 (2013), 1281-1338.

[48] A. M. Levin, M. A. Olshanetsky, A. V. Smirnov, A. V. Zotov, "Hecke transformations of conformal blocks in WZW theory. I. KZB equations for non-trivial bundles", SIGMA, 8 (2012), 37 pp., paper 095; 2012, 37 pp., arXiv: 1207.4386.

[49] A. M. Levin, M. A. Olshanetsky, A. V. Zotov, "Monopoles and modifications of bundles over elliptic curves", SIGMA, 5 (2009), 22 pp., paper 065; 2008 (v2 - 2009), 22 pp., arXiv: 0811.3056.

[50] M. F. Atiyah, "Vector bundles over an elliptic curve", Proc. London Math. Soc. (3), 7 (1957), 414-452.

[51] А. В. Зотов, А. М. Левин, "Интегрируемая система взаимодействующих эллиптических волчков", ТМФ, 146:1 (2006), 55-64; англ. пер.: A. V. Levin, A. M. Zotov, "Integrable model of interacting elliptic tops", Theoret. and Math. Phys., 146:1 (2006), 45-52.

[52] A. M. Levin, M. A. Olshanetsky, A. V. Smirnov, A. V. Zotov, "Characteristic classes of $\mathrm{SL}(N, \mathbb{C})$-bundles and quantum dynamical elliptic R-matrices", J. Phys. A, 46:3 (2013), 25 pp., 035201; 2012, 27 pp., arXiv: 1208.5750.

[53] E. Franco, O. Garcia-Prada, P. E. Newstead, Higgs bundles over elliptic curves, 2013, 41 pp., arXiv: 1302.2881.

[54] N. Hitchin, Higgs bundles and characteristic classes, 2013, 18 pp., arXiv: 1308.4603.

[55] D. Baraglia, L. P. Schaposnik, Real structures on moduli spaces of Higgs bundles, 2013, 19 pp., arXiv: 1309.1195.

[56] M.F. Atiyah, R. Bott, "The Yang-Mills equations over Riemann surfaces", Philos. Trans. Roy. Soc. London Ser. A, 308:1505 (1983), 523-615.

[57] V. G. Drinfeld, C. Simpson, "B-structures on $G$-bundles and local triviality", Math. Res. Lett., 2:6 (1995), 823-829.

[58] A. Beauville, Y. Laszlo, "Un lemme de descente", C. R. Acad. Sci. Paris Sér. I Math., 320:3 (1995), 335-340.

[59] D. Arinkin, S. Lysenko, "Isomorphisms between moduli spaces of $S L(2)$-bundles with connections on $\mathbb{P}^{1} \backslash\left\{x_{1}, \ldots, x_{4}\right\}$ ", Math. Res. Lett., 4:2 (1997), 181-190.

[60] D. Arinkin, S. Lysenko, "On the moduli spaces of SL(2)-bundles with connections on $\mathbf{P}^{1} \backslash\left\{x_{1}, \ldots, x_{4}\right\} "$, Int. Math. Res. Not., 1997:19 (1997), 983-999.

[61] A. M. Levin, M. A. Olshanetsky, A. Zotov, "Hitchin systems-symplectic Hecke correspondence and two-dimensional version", Comm. Math. Phys., 236:1 (2003), 93-133; 2001 (v3 - 2002), 39 pp., arXiv: nlin/0110045.

[62] P. Painlevé, "Sur les équations différentielles du second ordre à points critiques fixes", C. R. Acad. Sci. (Paris), 143 (1906), 1111-1117.

[63] Yu. I. Manin, "Sixth Painlevé equation, universal elliptic curve, and mirror of $\mathbf{P}^{2}$ ", Geometry of differential equations, Amer. Math. Soc. Transl. Ser. 2, 186, Amer. Math. Soc., Providence, RI, 1998, 131-151. 
[64] A. M. Levin, M. A. Olshanetsky, A. V. Zotov, "Painlevé VI, rigid tops and reflection equation", Comm. Math. Phys., 268:1 (2006), 67-103; 2005 (v2 - 2006), 32 pp., arXiv: math/0508058.

[65] A. Zotov, "Elliptic linear problem for Calogero-Inozemtsev model and Painlevé VI equation", Lett. Math. Phys., 67:2 (2004), 153-165; 2003, 13 pp., arXiv: hep-th/0310260.

[66] P. Boutroux, "Recherches sur les transcendantes de M. Painlevé et l'étude asymptotique des équations différentielles du second ordre", Ann. Sci. École Norm. Super. (3), 30 (1913), 255-375; suite, 31 (1914), 99-159.

[67] R. Garnier, "Étude de l'intégrale générale de l'équation VI de M. Painlevé dans le voisinage de ses singularités transcendantes", Ann. Sci. École Norm. Sup. (3), 34 (1917), 239-353.

[68] I. M. Krichever, "Isomonodromy equations on algebraic curves, canonical transformations and Whitham equations", Mosc. Math. J., 2:4 (2002), 717-752; 2001, 38 pp., arXiv: hep-th/0112096.

[69] C. T. Simpson, "Harmonic bundles on noncompact curves", J. Amer. Math. Soc., 3:3 (1990), 713-770.

[70] Э. Прессли, Г. Сигал, Группь петель, Мир, М., 1990, 456 с.; пер. с англ.: A. Pressley, G. Segal, Loop groups, Oxford Math. Monogr., Oxford Univ. Press, New York, 1986, viii+318 pp.

[71] В. Кац, Бесконечномерные алгебры Ли, Мир, М., 1993, 426 с.; пер. с англ.: V.G. Kac, Infinite-dimensional Lie algebras, 3rd ed., Cambridge Univ. Press, Cambridge, 1990, 400 pp.

[72] D. Arinkin, Moduli of connections with a small parameter on a curve, 2004, 19 pp., arXiv: math/0409373.

[73] В.И. Арнольд, Математические методы классической маханики, Наука, М., 1974, 431 с.; англ. пер.: V. Arnold, Mathematical methods of classical mechanics, Grad. Texts in Math., 60, Springer-Verlag, New York-Heidelberg, 1978, x+462 pp.

[74] И. М. Кричевер, “Метод усреднения для двумерных «интегрируемых» уравнений", Функи. анализ и его прил., 22:3 (1988), 37-52; англ. пер.: I. M. Krichever, "Method of averaging for two-dimensional "integrable" equations", Funct. Anal. Appl., 22:3 (1988), 200-213.

[75] K. Okamoto, "On the $\tau$-function of the Painlevé equations", Phys. D, 2:3 (1981), $525-535$.

[76] K. Takasaki, "Spectral curves and Whitham equations in isomonodromic problems of Schlesinger type", Asian J. Math., 2:4 (1998), 1049-1078; 1997, 41 pp., arXiv: solv-int/9704004.

[77] E. Looijenga, "Root systems and elliptic curves", Invent. Math., 38:1 (1976/77), $17-32$.

[78] И.Н. Бернштейн, О.В.Шварцман, "Теорема Шевалле для комплексных кристаллографических кокстеровских групп", Функи. анализ и его прил., 12:4 (1978), 79-80; англ. пер.: I. N. Bernstein, O. V. Schwarzman, "Chevalley's theorem for complex crystallographic Coxeter groups", Funct. Anal. Appl., 12:4 (1978), 308-310.

[79] J. Bernstein, O. Schwarzman, "Complex crystallographic Coxeter groups and affine root systems", J. Nonlinear Math. Phys., 13:2 (2006), 163-182.

[80] C. Schweigert, "On moduli spaces of flat connections with non-simply connected structure group", Nuclear Phys. B, 492:3 (1997), 743-755. 
[81] R. Friedman, J. W. Morgan, Holomorphic principal bundles over elliptic curves, 1998, 68 pp., arXiv: math/9811130.

[82] M.S. Narasimhan, C.S. Seshadri, "Stable and unitary vector bundles on a compact Riemann surface", Ann. of Math. (2), 82:3 (1965), 540-567.

[83] Н. Бурбаки, Группы и алгебры Ли. Группы Кокстера и системы Титса, группы, порожденные отражениями, системы корней, пер. с фр. А.И. Кострикина, А. Н. Тюрина, Мир, М., 1972, 334 с.; англ. пер.: N. Bourbaki, Lie groups and Lie algebras. Chapters 4-6, transl. from the 1968 French original by A. Pressley, Elem. Math. (Berlin), Springer-Verlag, Berlin, 2002, xii+300 pp.

[84] П. Дирак, “Лекции по квантовой механике”, прил. к кн.: Принципы квантовой механики, Наука, М., 1979, 408-475; пер. с англ.: Р. А. M. Dirac, Lectures on quantum mechanics, Belfer Grad. Sch. Sci. Monogr. Ser., 2, Academic Press, Inc., New York, 1967, v+87 pp.

[85] H. W. Braden, V. A. Dolgushev, M. A. Olshanetsky, A. V. Zotov, "Classical r-matrices and the Feigin-Odesskii algebra via Hamiltonian and Poisson reductions", J. Phys. A, 36:5 (2003), 6979-7000; 2003, 27 pp., arXiv: hep-th/0301121.

[86] P. Etingof, A. Varchenko, "Geometry and classification of solutions of the classical dynamical Yang-Baxter equation", Comm. Math. Phys., 192:1 (1998), 77-120.

[87] O. Schiffmann, "On classification of dynamical r-matrices", Math. Res. Lett., 5:1-2 (1998), 13-30.

[88] P. Etingof, O. Schiffmann, "Twisted traces of intertwiners for Kac-Moody algebras and classical dynamical r-matrices corresponding to generalized Belavin-Drinfeld triples", Math. Res. Lett., 6:5-6 (1999), 593-612; 1999, 18 pp., arXiv: math/9908115.

[89] L. Fehér, B. G. Pusztai, "Generalizations of Felder's elliptic dynamical $r$-matrices associated with twisted loop algebras of self-dual Lie algebras", Nuclear Phys. B, 621:3 (2002), 622-642; 2001, 22 pp., arXiv: math/0109132.

[90] O. Babelon, C.-M. Viallet, "Hamiltonian structures and Lax equations", Phys. Lett. B, 237:3-4 (1990), 411-416.

[91] E. Billey, J. Avan, O. Babelon, "The r-matrix structure of the Euler-Calogero-Moser model", Phys. Lett. A, 186:1-2 (1994), 114-118.

[92] K. Takasaki, "Painlevé-Calogero correspondence revisited", J. Math. Phys., 42:3 (2001), 1443-1473.

[93] Б. И. Сулейманов, "Гамильтоновость уравнений Пенлеве и метод изомонодромных деформаций”, Дифферени. уравнения, 30:5 (1994), 791-796; англ. пер.: B. I. Suleimanov, "The Hamilton property of Painlevé equations and the method of isomonodromic deformations", Differential Equations, 30:5 (1994), 726-732.

[94] A. Zabrodin, A. Zotov, "Quantum Painlevé-Calogero correspondence", J. Math. Phys., 53:7 (2012), 19 pp., paper 073507; 2011, 55 pp., arXiv: 1107.5672.

[95] A. Zabrodin, A. Zotov, "Quantum Painlevé-Calogero correspondence for Painlevé VI", J. Math. Phys., 53:7 (2012), 19 pp., paper 073508.

[96] A. Zabrodin, A. Zotov, Classical-quantum correspondence and functional relations for Painlevé equations, 2012, 38 pp., arXiv: 1212.5813.

[97] G. Aminov, S. Arthamonov, A. Smirnov, A. Zotov, Modifications of bundles as generating functions of Lax operators, Preprint ITEP-TH-27/13, 2013.

[98] G. Felder, C. Wieczerkowski, "Conformal blocks on elliptic curves and the KnizhnikZamolodchikov-Bernard equations", Comm. Math. Phys., 176:1 (1996), 133-161; 1994, 32 pp., arXiv: hep-th/9411004. 
[99] N. J. Hitchin, "Flat connections and geometric quantization", Comm. Math. Phys., 131:2 (1990), 347-380.

[100] G. Felder, "The KZB equations on Riemann surfaces", Symétries quantiques (Les Houches, 1995), North-Holland, Amsterdam, 1998, 687-725; 1996, 29 pp., arXiv: hep-th/9609153.

[101] D. A. Ivanov, "Knizhnik-Zamolodchikov-Bernard equations on Riemann surfaces", Internat. J. Modern Phys. A, 10:17 (1995), 2507-2536; 1994, 23 pp., arXiv: hep-th/9410091.

[102] N. Reshetikhin, "The Knizhnik-Zamolodchikov system as a deformation of the isomonodromy problem", Lett. Math. Phys., 26:3 (1992), 167-177.

[103] J. Harnard, "Quantum isomonodromic deformations and the Knizhnik-Zamolodchikov equations", Symmetries and integrability of difference equations (Estérel, PQ, 1994), CRM Proc. Lecture Notes, 9, Amer. Math. Soc., Providence, RI, 1996, 155-161; 1994, 9 pp., arXiv: hep-th/9406078.

[104] Л. Д. Ландау, Е. М. Лифшиц, Теоретическая физика, т. 3: Квантовая механика. Нерелятивистская теория, 3-е изд., Наука, М., 1974, 752 с.; англ. пер. 1-го изд.: L. D. Landau, E. M. Lifshitz, Course of theoretical physics, v. 3: Quantum mechanics: non-relativistic theory, Addison-Wesley Series in Advanced Physics, Pergamon Press Ltd., London-Paris; Addison-Wesley Publishing Co., Inc., Reading, MA, 1958, xii+515 pp.

[105] A. V. Zotov, "1+1 Gaudin model", SIGMA, 7 (2011), 26 pp., paper 067; 2010 (v2 2011), 26 pp., arXiv: 1012.1072.

[106] A. V. Zotov, "Classical integrable systems and their field-theoretical generalizations", Phys. Particles Nuclei, 37:3 (2006), 400-443.

[107] G. Aminov, S. Arthamonov, A. Levin, M. Olshanetsky, A. Zotov, Painlevé field theory, 2013, 67 pp., arXiv: 1306.3265.

[108] V. I. Arnold, B. A. Khesin, Topological methods in hydrodynamics, Appl. Math. Sci., 125, Springer-Verlag, New York, 1998, xvi+374 pp.

[109] В.И. Арнольд, "Гамильтоновость уравнений Эйлера динамики твердого тела и идеальной жидкости", УМH, 24:3(147) (1969), 225-226.

[110] B. Khesin, A. Levin, M. Olshanetsky, "Bihamiltonian structures and quadratic algebras in hydrodynamics and on non-commutative torus", Comm. Math. Phys., 250:3 (2004), 581-612.

[111] Н.Е. Жуковский, "О движении твердого тела, имеющего полости, наполненные однородной капельной жидкостью. I, II, III", Журнал Русского физико-химического общества, 17 (1885), 81-113, 145-199, 231-280.

[112] V. Volterra, "Sur la théorie des variations des latitudes", Acta Math., 22:1 (1899), 201-357.

[113] E. K. Sklyanin, "Separation of variables. New trends", Quantum field theory, integrable models and beyond (Kyoto, 1994), Progr. Theoret. Phys. Suppl., 1995, № 118, 35-60; 1995, 33 pp., arXiv: solv-int/9504001.

[114] А. В. Зотов, Ю. Б. Черняков, "Интегрируемые многочастичные системы, полученные с использованием предела Иноземцева", ТМФ, 129:2 (2001), 258-277; англ. пер.: А. V. Zotov, Yu. B. Chernyakov, "Integrable many-body systems via the Inosemtsev limit", Theoret. and Math. Phys., 129:2 (2001), 1526-1542; 2001, 16 pp., arXiv: hep-th/0102069.

[115] А. В. Смирнов, "Интегрируемые $s l(N, \mathbb{C})$-волчки как системы Калоджеро-Мозеpa", TMФ, 158:3 (2009), 355-369; англ. пер.: A. V. Smirnov, "Integrable $\operatorname{sl}(N, \mathbb{C})$ 
tops as Calogero-Moser systems", Theoret. and Math. Phys., 158:3 (2009), 300-312; Correspondence between Calogero-Moser systems and integrable $S L(N, \mathbb{C})$ Euler-Arnold tops, 2008, 14 pp., arXiv: 0809.2187.

[116] G. Aminov, S. Arthamonov, New $2 \times 2$-matrix linear problems for the Painlevé equations, 2011 (v2 - 2012), 18 pp., arXiv: 1112.4688.

[117] Г.А. Аминов, С.Б. Артамонов, "Вырождение эллиптической системы Шлезингера", ТМФ, 174:1 (2013), 3-24; англ. пер.: G. Aminov, S. Artamonov, "Degenerating the elliptic Schlesinger system", Theoret. and Math. Phys., 174:1 (2013), 1-20.

[118] A. Levin, A. Zotov, "On rational and elliptic forms of Painlevé VI equation", Moscow Seminar on mathematical physics. II, Amer. Math. Soc. Transl. Ser. 2, 221, Amer. Math. Soc., Providence, RI, 2007, 173-183.

[119] Э. Б. Винберг, А. Л. Онищик, Семинар по группам Ли и алгебраическим групnам, Наука, M., 1988, 344 с.; англ. пер.: A. L. Onishchik, È. B. Vinberg, Lie groups and algebraic groups, Springer Ser. Soviet Math., Springer-Verlag, Berlin, 1990, xx+328 pp.

[120] D. B. Fairlie, P. Fletcher, C. K. Zachos, "Infinite dimensional algebras and a trigonometric basis for the classical Lie algebras", J. Math. Phys., 31:5 (1990), 1088-1094.

[121] D. Mumford, Tata lectures on theta, v. I, Progr. Math., 28, Birkhäuser Boston, Inc., Boston, MA, 1983, xiii+235 pp.; v. II, Progr. Math., 43, 1984, xiv+272 pp.

[122] А. Вейль, Эллиптические функиии по Эйзенштейну и Кронекеру, Мир, М., 1978, 112 с.; пер. с англ.: A. Weil, Elliptic functions according to Eisenstein and Kronecker, Ergeb. Math. Grenzgeb., 88, Springer-Verlag, Berlin-New York, 1976, ii +93 pp.

[123] N. L. Gordeev, V.L. Popov, "Automorphism groups of finite dimensional simple algebras", Ann. of Math. (2), 158:3 (2003), 1041-1065.

\section{Андрей Михайлович Левин}

(Andrei M. Levin)

Лаборатория алгебраической геометрии,

НИУ "ВШЭ";

Институт теоретической и экспериментальной

физики

E-mail: alevin57@gmail.com

\section{Михаил Аронович Ольшанецкий}

\section{(Mikhail A. Olshanetsky)}

Институт теоретической и экспериментальной

физики;

Московский физико-технический институт

(государственный университет)

E-mail: olshanet@itep.ru

\section{Андрей Владимирович Зотов (Andrei V. Zotov)}

Математический институт им. В. А. Стеклова РАН;

Институт теоретической и экспериментальной физики;

Московский физико-технический институт (государственный университет)

E-mail: zotov@mi.ras.ru 